\title{
Abstracts of the International Medical Students Congress Sarajevo - SaMED 2017
}

\section{ORAL PRESENTATIONS}

01. Anatomical variations of human occipital condyles PAVLE BANOVIĆ', Bojana Krstonošić ${ }^{2}$

' University of Novi Sad, Medical faculty Novi Sad, Serbia, university of Novi Sad, Medical faculty Novi Sad, Department for anatomy, Serbia

Background: Occipital condyles, located at the inferior sides of lateral parts of occipital bone, are important structures that connect the cranium and the vertebral column. Their size, shape, location and, also, their congruence with superior articular facets of the atlas vertebra are of great importance for the stability of craniovertebral junctions. The progress in medical diagnostic and surgical techniques in the area of foramen magnum, requires the knowledge regarding anatomical aspects of this region. The purpose of this study was to evaluate the measurements of the occipital condyles, as well as to analyse the variations in the shape of the condyles and their position in relation to the foramen magnum. Methods: This study included 25 adult human skulls ( 11 male and 14 female) from the Osteological collection of the Department of Anatomy at Medical Faculty in Novi Sad. Nine parameters, which define morfological properties of the occipital condyles, were measured using Vernier caliper. Also, the shape and location of the condyles, as well as the narrowness of the foramen magnum were described. Results: Our findings show that length of the left occipital condyle, as well as distance between the posterior top of the left occipital condyle and basion are statistically greater in male crania, comparing to female crania. According to the shape of the occipital condyles, we classified them in seven groups. Predominant type is oval shaped. The condylar foramen is bilaterally present in $36 \%$ and the occipital condyles bilaterally protrude the foramen magnum in $40 \%$ of analyzed crania. Conclusions: Morphometric analysis of the occipital condyles showed variations in their size, shape, presence of condylar canal and relation to the foramen magnum.

Keywords: occipital condyle, foramen magnum, gender determination, morphometry, human skull.

02. Immunohistochemical Expression of Programmed Cell Death Ligand-1 in Non-Small Cell Lung Cancer PETRA PUPIĆ-BAKRAČ ${ }^{1}$, Nina Hanžič ${ }^{1}$, Koviljka Matušan $\|_{\text {lijaš2 }}^{2}$, Christophe Štemberger ${ }^{2}$, Ljiljana Bulat-Kardum², Aldo Ivančić ${ }^{2}$ Ksenija Lučin ${ }^{2}$

1 University of Medicine, Rijeka, Croatia, ${ }^{2}$ Clinical Hospital Center Rijeka, Croatia

Background: Programmed cell death ligand-1 (PD-L1) is a transmembrane protein expressed on different non-tumor as well as on tumor cells, serving them to evade detection and elimination by immune system. Lung cancer is the most common cancer in the world and non-small cell lung cancer (NSCLC) represents approximately $80 \%$ to $85 \%$ of all lung cancers. In the era of new molecular targeted therapies there is a constant need for the investigation of molecular substrate of cancer progression. Aim: To analyze the PDL-1 expression in NSCLC tumor cells (TC) and immune stromal cells (IC) and to compare it to usual clinicopathological parameters. Materials and Methods: Expression of PDL-1 was analyzed immunohistochemically in 110 surgically resected NSCLC tissue microarray samples, using Ventana ready-touse PD-L1 (SP263) primary antibody. The histological score (Hscore) was calculated separately on TC and IC, and was compared to clinicopathological parameters. Results: The expression of PDL-1 was seen in TC as well as in different types of IC as membranous and cytoplasmic staining of different intensity. The median TC Hscore was 8.12 (range 0-300), IC H-score was 14.04 (range 0-60) and they showed a significant correlation ( $\left.p=0.002, r_{p}=0.286\right)$. There was no significant difference in PDL-1 expression between different NSCLC histological types. Statistical analysis showed the association between higher level of $\mathrm{TC}$ and $\mathrm{IC}$ PDL-1 expression and higher histological grade $(p=0.002$ and $p<0.001$, respectively), higher pathological stage ( $p=0.011$ and $p=0.017$, respectively), and advanced clinical stage ( $p=0.046$ and $p=0.027$, respectively). Also, the level of TC PDL-1 expression correlated with the proliferative activity of tumor cells $\left(p=0.022, r_{p}=0.217\right)$, while higher level of IC PDL-1 expression showed the association with pleural infiltration $(p=0.026)$. Conclusion: Our results indicate that PDL- 1 may be involved in NSCLC progression, confirming its role as a target for PD-L1 inhibitors that showed benefits in clinical trials.

Keywords: Immunohistochemistry; Lung carcinoma, non-small cell Programmed cell death ligand-1; Progression.

03. Immunohistochemical analysis of a neuroendocrine tumor in liver: case report and literature review Ilhana Šestića', Sajra Vinčevića', Mirsad Babić2, Amir Spahić3

1 Faculty of Medicine, University of Sarajevo, Bosnia and Herzegovina, 2 Department of Pathology, Faculty of Medicine University of Sarajevo, Bosnia and Herzegovina, ${ }^{3}$ Hospital - Medical center Travnik, Department of Pathology, Travnik, Bosnia and Herzegovina

Introduction/aim: Neuroendocrine tumors arise from the cells of neuroectodermal origin, that are dispersed throughout the entire body. Gastrointestinal tract $(74 \%)$ is the most common origin of these tumors. The most commonly affected parts of gastrointestinal tract are the small intestine $(39 \%)$, appendix $(26 \%)$ and rectum (15\%). We report a case of a neuroendocine cancer in the liver. Case report: A 65-year-old man is hospitalized for an operative treatment of a tumor in the head of pancreas. He denies allergies to food and medication. Jaundice of skin was evident. The laboratory blood tests showed following abnormalities: high sedimentation rate $(80)$, high WBC count $\left(9,53 \times 10^{9} / \mathrm{L}\right)$, high MPV level $(10,6 \mathrm{fL})$, high PCT value $(0.35 \%)$, high glucose level $(10.2$ $\mathrm{mmol} / \mathrm{L})$ and high total bilirubin $(34.15 \mu \mathrm{mol} / \mathrm{L})$. Urine analysis showed presence of proteins and sugar. Total PSA level was increased (0.58); glucose level was $11.7 \mathrm{mmol} / \mathrm{L}$, glycohemoglobin $\mathrm{HbA} 1 \mathrm{C}-8.8 \%$; AST - $174 \mathrm{U} / \mathrm{L} ; \mathrm{ALT}-2541 \mathrm{U} / \mathrm{L}$, iron - $8 \mu \mathrm{mol} / \mathrm{L}$; chlorine $-96 \mathrm{mmol} / \mathrm{L}$

Immunohistochemical analysis showed positive staining for synaptopysin, chromogranin and neuron-specific enolase (NSE). Discretely positive staining for $\mathrm{CDX}_{2}$ in the liver parenchyma sugests that the tumor might originate from the appendix. Ki67 positivity indicates intermediate grade of the tumor. $\mathrm{CK}_{7}$ and $\mathrm{CK}$ 19-9 were negative in the tumor tissue. Hepatocyte antibody and TTF 1 were negative as well - which rules out the primary tumor in the liver or lungs. Conclusion: Using immunohistochemical markers, we proved the neuroendocrine origin of the neoplasm. Ki67 expression in the tumor cells confirmed the intermediate gradus of the neoplasm. The possibility of the primary neuroendocrine neoplasm of liver was ruled out.

Keywords: liver, neuroendocrine neoplasm, chromogranin, NSE

04. The Expression Rates of Estrogen and Progesteron Receptors Between Core Needle Biopsy and Surgical Specimens in Invasive Ductal Breast Cancer Goran Bokan', Nikolina Bosančić' ${ }^{\prime}$, Ljiljana Tadić-Latinović $\mathrm{MD}, \mathrm{PhD}^{2}$

'University of Banja Luka, Faculty of Medicine, Banja Luka, Bosnia and Herzegovina, 2Department of Pathology, University Clinical Center of Republic of Srpska, Bosnia and Herzegovina 
Introduction: Majority of breast cancer (70-80\%) belongs in the group of invasive ductal carcinoma which is usually connected with ductal carcinoma in situ (DCIS). The status of ER, PgR and HER2 is critical in the management of patients with this type of malignancy. ER is a powerful predictive factor for response to endocrine treatment and long-term outcome. Aim: The aim of this study is to explain the concordance between the ER and PgR receptor status, including immunohistochemistry (IHC) and FISH analyses assessment scores in paired core needle biopsy and surgical tissue selections of invasive ductal breast cancer. Materials and methods: We retrospectively examined 50 female patients with breast carcinoma without neoadjuvant chemotherapy who underwent CNB and surgical resection at the Department of Pathology, in University Clinical Center of Republic of Srpska, Banja Luka. ER and PgR were determined by IHC using the SP1 antibody (DAKO) and PgR (PgR 636, DAKO). Nuclear staining graded from o to 8 using the Allred score. Scores of $>2$ were considered positive. Results: The median age of patients was 58 (28-81) years. $50 \%$ patients received radical mastectomy followed by lymphadenectomy. ER was scored as positive in $38(76 \%)$ of the CNB and negative in $12(24 \%)$. In the surgical specimens, ER was positive in $36(72 \%)$ of the cases and negative in $14(28 \%)$. Evaluation of ER expression on CNB samples had a $90 \%$ concordance rate with ER results on surgical specimens, with good overall agreement $(\mathrm{K}=0.733)$. For $\mathrm{PgR}$ in the $\mathrm{CNB}, 72 \%$ were scored as positive compared with $68 \%$ in the surgical specimens. PgR expression on CNB samples also revealed good agreement with those on surgical specimens; the overall concordance rates was $88 \%$, with $\mathrm{k}$ value of 0.702 , respectively. Conclusions: The expression rates of ER and PgR showed no significant difference between CNB and surgical specimens, so we suggest that CNB should be considered the initial procedure to assess receptor status in IDC.

Keywords: Breast cancer, estrogen receptor, progesterone receptor, IHC, FISH.

05.

\author{
Abdominal endometriosis \\ ALEKSANDRA KOVACIC \\ University of Novi Sad, Medical faculty Novi Sad, Serbia
}

Background: Endometriosis is the condition which is characterised by the presence of endometrial tissue outside the uterine cavity. It is most likely to be found in ovaries, uterosacral and big ligament, Fallopian tubes and intestinal tract. The most common sites of the intestinal endometriosis are rectosigmoid junction and small bowel. Ectopic endometrium can be found in all layers of the intestine wall, but it usually affects subserosa and serosa. Aim: To analyse avaliable data of patohystological verified endometriosis in intestinal tract (age, location, clinical and patohystological diagnosis, other diseases and conditions) and to compare results with the results from other scientific works. Material and Methods: Retrospective study contained 16 patient with confirmed patohystological diagnosis of endometriosis in 5 years. Data are presented in table and charts. Patohystological samples were photographed and microphotos were described and inclosed with charts. Results: The highest incidence of endometriosis ( $56 \%$ ) is among the patients between age $31-40$, followed by group of patients between age 41-50 with percentage frequency of $25 \%$ When it comes to the most common sites of endometriosis, we talk about anterior abdominal wall (33\%) and ovaries (28\%), while the intestinal tract is affected in less than $5 \%$ of cases. In most cases clinical diagnosis is matching patohistological and endometriosis is not followed by additional diseases or conditions Conclusion: Our results and the results from other scientific works match when it comes to age.

Keywords: Endometriosis; intestinal tract; age; locations.

06.

Increased Number of CD34- And CD105- Positive Blood Vessels And Capillaries In The Uninvolved Rectal Mucosa $10 \mathrm{~cm}$ And $20 \mathrm{~cm}$ Away From The Malignant Tumor

Stefan Maksimović1, Sanja Despotović2 ${ }^{2}$ Ivana Lalić ${ }^{2}$
7 School of Medicine Belgrade, University of Belgrade, 11000 Belgrade, Serbia, ${ }^{2}$ Institute of Histology and Embryology, School of Medicine, University of Belgrade, Serbia

Background: Despite numerous data on the importance of the interaction of tumor cells and tumor-associated stromal elements in the progression of malignant disease, little is known about changes of the uninvolved, healthy-looking mucosa around the tumor. The aim of our study was to visualize and quantifiy blood vessels and capillaries in the lamina propria at a distance 10 and $20 \mathrm{~cm}$ away from the rectal adenocarcinoma and to compare it with the rectal lamina propria of healthy patients. Methods: Samples of the rectal mucosa 10 and $20 \mathrm{~cm}$ away from the rectal adenocarcinoma were endoscopically collected during diagnostic examinations of 5 patients. Control samples were obtained from healthy patients $(n=5)$ in the same institution. Intestinal tissue was fixed in $10 \%$ formalin and embedded in paraffin. Sections 3-5 microns thick were immunihistochemically stained with CD34 and CD105 antibodies. For statistical analysis program SPSS for Windows 15.0 was used. Results: In the lamina propria at a distance $10 \mathrm{~cm}$ away from the tumor both the number of $\mathrm{CD}_{34}$ and CD105- positive blood vessels was significantly increased compared to the lamina propria of patients without malignant tumors $(p<0.01)$ and at a distance $20 \mathrm{~cm}$ from the tumor $(p$ 0.01 ). At a distance $20 \mathrm{~cm}$ away from malignant tumors there is a significantly greater number of CD105- positive blood vessels in comparison with the lamina propria of healthy persons $(p<0.01)$. Conclusion: In the rectal lamina propria 10 and $20 \mathrm{~cm}$ away from the malignant tumors there is significantly increased number of both CD34- and CD105- positive blood vessels and capillaries.

Keywords: adenocarcinoma, rectum, blood vessels.

Oral session II:

Epidemiology, Biochemistry, Physiology

07. Determination of Risk Factors for Hydatid Disease among Animal Breeders in Dinar-Afyonkarahisar

Mehmet Fatih Aydin', Hakan Güzel ${ }^{2}$

' Faculty of Health Sciences, Karamanoglu Mehmetbey University, Turkey, 2 District Directorate of Agriculture, Dinar-Afyonkarahisar 03400, Turkey

Background: Hydatid disease is a zoonotic parasitic infection caused by a tapeworm of called Echinococcus granulosus. It was aimed with this study to determine the risk factors for hydatid disease among animal breeders in Dinar-Afyonkarahisar province of Turkey. Methods: Between July 2015 and May 2016 a questionnaire form including risk factors for hydatid disease were applied by face to face interviews to 95 individuals. Survey data were analysed by percentage. Results: Average of age was calculated as 44.87 . It was determined that $45.26 \%$ of animal breeders have no information about hydatid cyst. $38.94 \%$ of animal breeders reported that they don't administer antiparasitic drug to animals. $68.42 \%$ of them stated that they will not be able to recognize hydatid cyst while slaughtering. $24.21 \%$ of individuals said that they saw hydatid cyst while slaughtering. $80.00 \%$ of animal breeders stated that they have no information about transmission of the disease. $2.10 \%$ of participants reported that there are some persons who caught hydatid disease around them. Most of the participants $(56.84 \%)$ indicated that infected organs should be given to dogs or put in the rubbish box. $50.52 \%$ of the individuals reported that their dogs can enter easily to barns and they give giblets to dogs. Also $57.89 \%$ of animal breeders specified they don't give antiparasitic drug to their dogs. Conclusion: It was detected that the animal breeders in DinarAfyonkarahisar have insufficient information for some subjects for the hydatid disease and also, we detected some risk factors for the disease.

Keywords: Hydatid disease, Animal breeder, Risk factor, Dinar, Turkey. 
08. Prevalence of Obesity among Medical Students in Belgrade, Serbia

MAŠA JOSIPOVIĆ', Aleksa Jovanović", Darija KisićTepavčevićs , Gorica Marić ${ }^{2}$

School of Medicine Belgrade, University of Belgrade, 11000 Belgrade, Serbia, 2 Institute of Epidemiology, School of Medicine, University of Belgrade, Serbia

Background: Obesity has become a leading global health problem, reaching epidemic proportions, and it has increasing trends among the young $(1,2)$. The aim of this study was to estimate the prevalence of obesity on a sample of medical students at University of Belgrade, Serbia. Methods: This cross-sectional study included 249 fourth-year students at School of Medicine, University of Belgrade. Data was collected using an anonymous questionnaire. Body mass and body height were measured in order to determine the participants' Body Mass Index (BMI). Obtained data was analyzed using descriptive statistics and Spearman correlation coefficient. Results: The study sample comprised of $36.9 \%$ males and $63.1 \%$ females. Average age was $22.5 \pm 1.3$ years. A total of $9 \%$ students were on a hypocaloric diet. According to BMI, the majority of participants $(74.5 \%)$ were in normal body mass range, $18.1 \%$ were overweight and obese, while $7.4 \%$ were underweight. On the other hand, self-assessment of body mass revealed that some participants were unaware of their obesity or underweight, perceiving themselves as having a normal range of body mass. Obesity status significantly correlated with lower physical appearance satisfaction $(\rho=-0.280, p<0.001)$ and lower self-esteem $(\rho=-0.138, p=0.035)$. There was also a statistically significant correlation of higher $\mathrm{BMI}$ with male gender $(\rho=-0.326, p<0.001)$. Conclusion: Results of this study showed high prevalence of obesity among medical students at School of Medicine, University of Belgrade. Aside from affecting physical health, obesity also has an impact on mental health, by lowering self-esteem and physical appearance satisfaction. These findings are alarming because these students, as future physicians, should be aware of importance of having and promoting a healthy lifestyle and therefore there is a need for implementation of preventive and treatment programs.

Keywords: Obesity; BMI; Students; Prevalence.

09. Serum Homocysteine Levels in Patients with Probable Vascular Dementia

Azra Mujkic', Elma Ramakic

Faculty of Medicine, University of Sarajevo, Bosnia $\circlearrowright$ Herzegovina

Backround: Elevated plasma homocysteine (Hcy) levels have been associated with vascular dementia (VD) and cognitive impairment. Studies have shown that Hcy may have direct and indirect neurotoxicity effects. The aim of the study was to investigate serum Hcy concentration in patients with probable VD and in agematched controls. We also aimed to determine whether there is an association between serum tHcy concentration and cognitive impairment in patients with probable VD. Methods: Serum concentration of total Hcy was determined by the Fluorescence Polarization Immunoassay on the AXSYM System. Cognitive impairment was tested by the MMSE score. Results: Body mass index (BMI) was calculated for each subject included in the study. Age, systolic, diastolic blood pressure and BMI did not differ significantly between the two groups. Mean serum tHcy concentration in the control group of subjects was $13.35 \mu \mathrm{mol} / \mathrm{L}$, while in patients with probable VD the mean serum tHcy concentration was significantly higher than $19.45 \mu \mathrm{mol} / \mathrm{L}(\mathrm{p}=0.002)$. A negative but not significant association between serum tHcy concentration and cognitive impairment tested by the MMSE score in patients with probable VD was determined. Conclusions: Increased tHcy concentration in patients with probable VD suggests that there is a possible independent role of Hcy in the pathogenesis of VD.

Keywords: vascular dementia, homocysteine, cognitive impairment, Mini-Mental State Examination.
10. Characteristics of dyslipidemia in patients with End Stage Renal Disease

Mervana Halilović', Jasmina Hakmi', Emina Kiseljaković ${ }^{1}$ Amela Dervišević1, Amela Bećiragić

7 Faculty of Medicine, University of Sarajevo, Bosnia and Herzegovina, ${ }^{2}$ Clinic of Hemodialysis, Clinical Center University of sarajevo, Sarajevo, Bosnia 0 Herzegovina

Background: Dyslipidemia is metabolic disorder which appears with elevation of plasma cholesterol, triglycerides (TGS) and a low high-density lipoprotein (HDL) level that contributes to the development of atherosclerosis. Patients on hemodialysis (HD) have high prevalence of dyslipidemia, high rate of death due to atherosclerotic vascular disease. Traditional risk factors such as disturbance of lipid parameters are responsible for development of atherosclerosis. The aim of study was to examine characteristics of dyslipidemia in patients on hemodialysis. Methods: Cross-sectional study included 97 subjects, divided into two groups; 59 patients on HD $(49,53 \pm 11,34$ years), both genders $(f=37, m=22)$, recruited from Clinic for hemodialysis Clinical Center University of Sarajevo, and 38 apparently healthy subjects $(49,18 \pm 15,20$ years; $f=25$, $m=13$ ), designated as a control group (CG). TC, TCS, HDLC, lowdensity lipoprotein cholesterol (LDLC), very low-density lipoprotein cholesterol (VLDLC), C-reactive protein (CRP) and albumin values were obtained by standard laboratory methods. Atherogenic index of plasma (AIP $=\log (\mathrm{TC} / \mathrm{HDL}-\mathrm{C})$ was calculated. Results: HD patients had higher levels of $\operatorname{LDLC}(2,59 \pm 0,97 \mathrm{mmol} / \mathrm{l}$ vs. $2,58 \pm$ $0,67 \mathrm{mmol} / \mathrm{l}), \operatorname{VLDLC}(0,78 \pm 0,35 \mathrm{mmol} / \mathrm{l}$ vs. $0,70 \pm 0,32 \mathrm{mmol} / \mathrm{l})$, TC $(1,83 \pm 1,08 \mathrm{mmol} / \mathrm{l}$ vs. $1,51 \pm 0,68 \mathrm{mmol} / \mathrm{l})$ and AIP $(0,23 \pm 0,28$ $\mathrm{mmol} / \mathrm{l}$ vs. $0,14 \pm 0,29 \mathrm{mmol} / \mathrm{l})$ compared to CG ( $p>0.05)$. No difference in TC between two groups was observed. The values of HLDC and albumin were significantly lower in HD comparing to control group $(0.91 \pm 0,24 \mathrm{mmol} / \mathrm{l}$ vs. $1,04 \pm 0,31 \mathrm{mmol} / \mathrm{l} ; \mathrm{p}=0,03$, and $36,93 \pm 3,54 \mathrm{~g} / \mathrm{l}$ vs. $42,10 \pm 2,63 \mathrm{~g} / \mathrm{l}$; $\mathrm{p}<0,0005$; respectively) The CRP values were higher in $\mathrm{HD}$ patients comparing to healthy subjects $(3,30 \quad(1,00-7,70)$ vs. $1,40 \quad(0,55-2,35) ; \quad$ p $<0,0005)$. Conclusion: HD patients have expressed characteristics of dyslipidemia - significantly decreased levels of HDLC and higher level of cholesterol rich lipoproteins.

Keywords: dyslipidemia, end stage renal disease (ESRD), hemodialysis.

11. Dimerization and diffusion parameters of RAR and RXR nuclear receptors: a combined biophysical study Bálint Rehó ${ }^{1}$ György Vámosi ${ }^{1}$

1 Department of Biophysics and Cell Biology, University of Debrecen Faculty of Medicine, Debrecen, Hungary

Background: Nuclear receptors are transcription factors that are able to regulate gene expression in a ligand dependent manner. They play a central role in cell differentiation, growth, and death. We investigated interactions and dynamics of retinoic acid receptor (RAR) and retinoic $X$ receptor (RXR) acting in a dimer in living cells. Their operation is described by the nuclear receptor molecular switch model. In the absence of ligand receptors are bound to DNA associated with a corepressor complex, and repress transcription. In the presence of agonist receptors change their conformation, and the corepressor complex is replaced by coactivator complexes resulting in gene transcription. Nowadays, this model is being changed for a more dynamic one due to intense investigations in the field. In our studies we wanted to determine the affinity of the receptors to each other in the absence and the presence of agonist ligands. Methods: We transfected HEK293T cells with nuclear receptors marked by fluorescent proteins (GFP, mCherry). We monitored changes in mobility by fluorescence correlation spectroscopy (FCS). The changes in the dimerization of these molecules is determined by fluorescence resonance energy transfer (FRET). Both method capable of following the dynamics of fluorescent molecules with high sensitivity in living cells. Results: We revealed that agonist treatment decreased the mobility of RAR or RXR molecules transfected alone. Results showed that co-transfection of the two receptors decreases their mobility even in the absence of ligand. Our FRET results showed that the heterodimerization is increased in the presence of RXR ligand. This method can be used for the 
investigation of RXR homodimerization of marked RXR receptors. In the presence of RXR ligand, homodimerization is also increased. Conclusion: Our FCS studies combined with FRET measurements are described a complex system and prove the central role of the $\mathrm{RXR}$ in the activation of nuclear receptors.

Keywords: nuclear receptors, rar, rxr, FCS, flow cytometry.

12. The Impact of Tobacco Smoking on Disease Outcome in Rheumatoid Arthritis Patients

ADEM CEMERLIC', Amela Dervišević ${ }^{2}$

Faculty of Medicine, University of Sarajevo, Bosnia and Herzegovina, 2 Department of Human Physiology, Faculty of Medicine, University of Sarajevo, Bosnia and Herzegovina

Background: Rheumatoid arthritis (RA) is an autoimmune, progressive, chronic systemic disease characterised by nonspecific inflammation and the destruction of bones and joints. It is a multifactorial disease that results from interactions between genetic and environmental factors. Tobacco smoking is the major environmental risk factor, whose role in the occurrence and severity of RA is still unclear. The aim of the present study was to analyse the relationship between tobacco smoking and disease outcome in RA patients. Methods: The study included 67 RA patients who were divided into 2 groups according to their smoking status: exposure (ever, onset, and current) to smoking (41 patients) and not exposed to smoking (26 patients). The following laboratory and clinical parameters were analysed: age, disease duration, erythrocyte sedimentation rate (ESR), rheumatoid factor (RF), C-reactive protein (CRP), morning stiffness duration, number of tender joints (TJC), number of swollen joints (SJC) and visual analogue scale (VAS) pain score. Results: The mean disease duration was significantly higher in RA patients exposed to smoking than in those RA patients not exposed to smoking ( $11.5 \pm 8.9$ years vs. $7.3 \pm 5.9$ years, respectively, $p=0.045)$. SE and CRP were significantly higher in RA patients exposed to smoking than not exposed to smoking RA patients $(p=0.001$ and $p<0.0005$ respectively). The exposure to smoking RA patients also had higher TJC $(p=0.008), S J C(p=0.03)$ and VAS pain score $(p<0.0005)$. In exposure to smoking RA patients, a statistically significant positive correlation was observed between SE and smoking duration $(r=0.529, p<0.0005)$. Conclusion: Smoking tobacco products has an effect on the progression of rheumatoid arthritis and is also linked to worse outcomes. Thus, tobacco smoking cessation should be one of the main parts of the disease treatment process in rheumatoid arthritis patients.

Keywords: Rheumatoid arthritis; Tobacco smoking; Disease outcome.

13. The Relationship Between Sleep Habits and Academic Performance In Medical Students In Bosnia And Herzegovina

AMILA MUHIC ${ }^{1}$, Adin Džanko ${ }^{2}$, Lejla Hadžijusufovićc', Amela Dervišević3 ${ }^{3}$ Nesina Avdagić3 ${ }^{3}$, Nermina Babić 3

1 Faculty of Medicine, University of Sarajevo, Bosnia and Herzegovina, 2 Emergency Medical Service of the Sarajevo Canton, Sarajevo, Bosnia and Herzegovina, 3 Department of Human Physiology, Faculty of Medicine, University of Sarajevo, Bosnia and Herzegovina

Background: The quality of sleep has significant effects on cognitive performance of students and a positive effect on the process of remembering new information. Due to academic requirements, medical students are exposed to big amounts of stress which has an impact on sleep quality. The aim of this study was to evaluate sleep habits of medical students and the relationship between sleep habits and academic performance. Methods: A cross-sectional study was conducted at Faculty of Medicine, University of Sarajevo. The study included 200 students who were divided into two equal groups: students of the second year $(n=100,39$ males, 61 females, mean age 21) and students of the sixth year ( $n=100,37$ males, 63 females, mean age 28). Students fulfilled a self-administered "Morningness-Eveningness questionnaire" containing 19 questions and based on the final number of points, students were divided into five subgroups due to circadian rhythm. Academic performance was assessed by students' grade point average. Statistical analysis was done using SPSS 13.0. Values of $p<0.05$ were considered statistically significant. Results: Average grades did not significantly differ ( $p=$ 0.608 ) between second and sixth year students. There was a statistically significant difference $(p=0.001)$ between the circadian rhythm of second and sixth year medical students. Regardless of the year of study, the most common circadian rhythm types were intermediate, moderate morning and moderate evening type and the least frequent were definitely morning and definitely evening type. Statistically significant positive correlation (rho $=0.419 ; \mathrm{p}$ 0.0005 ) was found in sixth-year students' circadian rhythm and academic performance. Conclusion: The second-year medical students with the best academic success are moderate evening type of circadian rhythm and the sixth-year students with the best academic success are intermediate type of circadian rhythm.

Keywords: academic performance, medical students, sleep habits.

\section{The ratio of fractal and textural parameters of chromatin} peripheral blood neutrophils

MUJEZINOVIĆ NEJIRA ', Marković Danilo

School of Medicine, University of Belgrade, Belgrade, 11000 Serbia Background: Fractal and textural analysis are two mathematical algorithms that is in cell biology are often used to evaluate structural changes in nuclear chromatin during various physiological and pathological processes. The interrelation of the parameters of these two methods is unknown. The aim of ou research was to determine the existence and strength of the correlation between fractal and textural parameters in a sample of peripheral blood neutrophils treated using DNA-specific staining. Methods: We analyzed the chromatin of 30 neutrophil granulocytes of peripheral blood neutrophils treated with specific Feulgen staining. Using the special software of the National Institutes of Health (NIH, USA), and MATLAB programming code, for each chromatin structure we determined seven parameters of fractal and textural analysis: fractal dimension, lacunarity, angular second moment, inverse difference moment, textural variance, textura contrast, textural correlation and entropy. Results: We found a statistically significant positive correlation between chromatin entropy and fractal dimension $(r=0.45 ; p<0.01)$. With the growth of entropy, increased the value of the fractal dimension, and vice versa, with the decline in the value of entropy, the value of chromatin fractal was also reduced. Between the value of the inverse difference moment (indicator of textural homogeneity) and the fractal dimension of chromatin, we found a statistically significant negative correlation $(r=-0.49, p<0.01)$. Conclusion: There is a significant relationship between some fractal and textural parameters of chromatin in peripheral blood neutrophils. Fractal and textural algorithms are statistically complementary and can be successfully integrated into a common protocol for the mathematical analysis of structural changes in chromatin in physiological conditions.

Keywords: entropy, homogeneity, complexity, DNA

Oral session III:

Surgery

15. The Frequency of Breast Conserving and Radical Mastectomy in Clinical Center University of Sarajevo in Comparison to More Developed Countries Pidro A ${ }^{1}$, Prnjavorac L ${ }^{1}$, Đozić A ${ }^{1}$, Salibašsić $M^{2}$ 1 Faculty of Medicine, University of Sarajevo, Bosnia and Herzegovina, ${ }^{2}$ Clinical Center University of Sarajevo, Clinic for glandular oncology, Bosnia and Herzegovina

Background: Breast cancer is the most common invasive cancer in females worldwide. There are different types of treatment for this disease, but most of the patients undergo some kind of a surgery at one point. The surgeries can be breast conserving or radical mastectomy, with or without axillar lymph node dissection. Materials and methods: A retrospective study was conducted on 
Clinic for surgical oncology in Clinical center University of Sarajevo using the information from patients' histories in 6 month period. There were 69 patients, mean age 59.94 (min 32, $\max 79$ ). Another five similar studies were found where the statistical data was collected and used for comparison. Results: Results showed that $34,78 \%$ patients had breast conserving mastectomy, of which $91,67 \%$ had axillary dissection. Radical mastectomy was done in $65,22 \%$ patients, of which $93,33 \%$ had axillary dissection. There were $58,46 \%$ patients who had axillar lymph node dissection despite negative lymph nodes. Mean frequencies for conserving versus radical mastectomy in five other studies were $54,29 \%$ and $45,71 \%$ retrospectively. Only one study had slightly frequent radical surgery $(51,77 \%)$. Conclusion: Patients who underwent radical surgery were much more frequent comparing to the patients with breast conserving surgery. Almost all patients had axillary dissection despite half of them having negative lymph nodes. Comparing to these results, four out of five other researches from more developed countries showed opposite results with more frequent breast conserving surgeries.

Keywords: Breast cancer; Mastectomy; Axillary dissection

\section{Analysis of trends in orthopedic prosthetics}

Serhiy Hovornyan', Adelaide Antunes², Alena Antoniv' Marcelo $\mathrm{Kropf}^{3}$

'Bukovinian State Medical University, Chernivtsi, Ukraine, ${ }^{2}$ Federal University of Rio de Janeiro, Rio de Janeiro, Brazil, 3 Instituto Nacional de Traumatologia e Ortopedia Jamil Haddad, Rio de Janeiro, Brazil

Introduction: Obtaining technological information is crucial for build knowledge and support the development and production of new low-cost healthcare technologies.The aim is to analyze the technological information contained in scientific articles and patents with a view to identifying current and future technological trends for cheap orthopedic implants. Methodology: We retrieved and analyzed data on patent applications using the Derwent Innovations Index and accessed the Web of Science database to find scientific articles. The period of study was 2000 to 2014. It is essential to treat the data retrieved from patent documents and scientific articles to extract the technological information they contain and group it according to the technologies described.

Results: Using the methodology, a total of 22,615 patents were retrieved. Using the adjustment criteria, we retrieved a total of 8,688 articles indexed on the Web of Science database between 2000 and 2014. The classification of the groups of technologies described in the patents and articles yielded a taxonomy of trends that covers all the technologies identified. (Bio)mechanical and orthopedic engineering technologies $(60 \%)$, (nano)biomaterials technologies (20\%), and (bio)chemical and (bio)pharmaceutical technologies $(16 \%)$ represent the current trends in orthopedic prosthetics. The last two categories correspond to the application of nanobiotechnology to new biomaterials for implants (32\%) and indicate the future trends in orthopedic prosthetics. Just $4 \%$ relate to technologies for testing and diagnosis. Conclusion: The orthopedic prosthetics is at a transitional moment, as displayed by the technological information retrieved and analyzed. The implants developed using nanobiotechnology represent the cutting edge of devices available at the present time. The focus is now on the research and development of materials than tissues. The use of scaffolds for the growth and differentiation of stem cells in bone tissue and genetic modification of tissue are the key technologies being developed in the field of biotechnology

Keywords: orthopedic prosthetics, nanobiotechnology, bone tissue

17. Assessment of effects of pentazocine, noraminphenzon and ketoprophen on treatment of postoperative pain after total hip arthroplasty and hip osteosynthesis

Andjela Stojanovic', Stefan Djurdjic

' Faculty of Medicine Foča, University of East Sarajevo, Bosnia and Herzegovina.
Background: Despite the application of modern surgical and analgesic protocol after a hip replacement surgery, the problem of pain has not been adequately solved yet. The aim of this study is to compare the effects of ketoprophen, normaniphenazon and pentazocine on suppression of postoperative pain after a total hip arthroplasty and hip osteosynthesis, according to the assessment of pain intensity. Methods: This is a retrospective clinical study which includes 53 patients aged 58 years who were operated at University Hospital in Foča. Patients were divided into three groups: A - 17 patients who were administred ketoprophen, B - 18 patients who were administred noraminophenazon, $C$ - 18 patients who were administred pentazocine. After previously mentioned surgeries, we assessed the intensity of postoperative pain at rest as well as the effects of analgesics, administered in the first 48 hours using the visual analog scale (VAS) from 0 - "no pain" to 10 - "worst possible pain." Results: After the surgical procedure at the time when patients required analgesia they showed pain intensity VAS 7. Half an hour after the administration of analgesics, pain average value was average 3.5 VAS. Comparing the effects of different analgesics, we found a statistically significant difference ( $p<0.05)$. After the application of ketoprophen patients felt the least pain -3.4, after noraminophenazon-3.9 and after pentazocine3.7. After the second requirement for analgesia, ketonal was again the most effective: ketoprophen 3.0, pentazocine 3.9, noraminophenazone 3.7. More than $40 \%$ of patients felt nausea after the administration of pentazocine. In three patients, noraminofenazon could not independently relieve pain and tramadol was additionally administered. Conclusion: Ketoprophen was the most efficient in reduction of postoperative pain. It was administered in two doses and could relieve postoperative pain itself. Pentazocine was more efficient compared to noraminophenazon.

Keywords: analgesics, postopertive pain, hip surgery, assessment

\section{Congenital facial clefts of lip and palate, multidisciplinary} approach

Amer Kubat', Emrah Gačić ${ }^{1}$, Emir Kalbić1, Enita Nakaš

1 Faculty of Dentistry, University of Sarajevo, Bosnia and Herzegovina, ${ }^{2}$ Faculty of Medicine, University of Sarajevo, Bosnia and Herzegovina

Background: Cleft lip and palate are congenital malformations. The etiology of cleft is very complex. Thus including genetic and environmental triggers. The diagnosis can be made before and after birth. Intrauterine diagnosis can be established using 3D ultrasound examination. A final diagnosis is made at birth. Therapy of patients with cleft lip and palate does not comprise only surgical closure of the cleft - it requires multidisciplinary approach: including dental treatment, speech therapist, pediatrician etc. With the aim to achieve aesthetically and functionally optimal results. Methods: This is a case report of the boy and the girl. The boy referred to orthodontic department school of dental medicine the University of Sarajevo. First as a newborn baby for Phase I of treatment. Which include active appliance for correcting of fragments prior surgery. And now he is in the II phase of the treatment. This phase of the treatment is aiming in improving skeletal relationship as well as occlusion, and aesthetic. After completion of full permanent dentition, patient will be treated with full fixed appliances followed by MFH surgery for final correction of nose and palate. Results: Full reconstruction of the oral functions ( functional occlusion, speech) and pleasant esthetic appearance of the face. Surgically closed cleft lip and palate, successful therapy from doctors of pediatrics, ORL and speech therapy. At last, successful dental therapy with ortodontic treatment. That is the circle of multidisciplinary approach, which show a bond between the doctors and that is critical to success. Conclusion: After all functionality, aesthetic results and satisfied patient are product of the multidisciplinary approach.

Keywords: Cleft lip and palate, multidisciplinary approach, successful therapy, functional and aesthetical results, satisfied patient. 
19.

\section{Giant Struma Ovari}

DI NARDO NICOLA', Molnar Anca-Alexandra', Luca DianaAdriana 'Butiurca Vlad-Olimpiu

University of Medicine and Pharmacy of Targu Mures, Targu Mures, Romania

Background: Struma ovarii is a tumor rarely encountered in medical practice. Histologically, it is a form of mature teratoma in which more than $50 \%$ of the tumor consists of thyroid tissue, affecting mainly patients in the 5th and 6th decade. It amounts to $1 \%$ of all ovarian neoplasms, and $95 \%$ of the cases are benign tumors. Symptoms, present in only $5 \%$ of the cases, consist in: pelvic tumor, ascites, Meigs syndrome, and hyperthyroidism. Preoperative diagnosis, based on imaging, hormone levels assessment and tumor markers, is difficult, and in most cases the diagnostic is established postoperatively, following histopathologic examination. Methods: This is the case of a 47-year old patient admitted in the Surgery Clinic No. I of the Tîrgu Mureş Emergency Clinical County Hospital presenting: massive abdominal tumor, dyspnea, subocclusive syndrome. Clinical and ultrasound preoperative gynecologic examination revealed a giant pelvicabdominal cystic tumor possibly arising from the right ovary. Due to the large size of the tumor, laparoscopic exploration failed. Subsequent exploratory laparotomy consisted of: right adnexectomy, splenectomy, omphalectomy, extended abdominal parietal resection with single-layer abdominoplasty, drainage. The intraoperative diagnosis was giant right ovarian multilocular cystic compressive tumor $(15,000 \mathrm{ml})$, adherence syndrome, fundic extracavitary pedunculated uterine fibroma, ascites, portal hypertension with umbilical vein repermeabilization, umbilical hernia. Results: The postoperative evolution was complication-free, intestinal transit resumed in the 3 rd postoperative day and the patient was discharged after 11 days. Histopathologic examination revealed struma ovarii. Conclusions: The preoperative diagnosis of a giant cystic tumor must include the possibility of struma ovarii, hence endocrinologic exploration (of thyroid hormones) is necessary. The slow evolution and the low metastatic potential of this tumor indicate a conservative surgical treatment, especially in young patients. The lack of a "gold standard" in diagnosis and treatment is an argument for carrying out further studies regarding this pathologic entity.

Keywords: Giant struma ovarii; Hyperthyroidism; Histopathological examination; Abdominoplasty

\section{Ileocecal Intussusception Associated with Gastrointestinal Stromal Tumor in Young Woman. NATALIA KULIKOVA' \\ Moscow Clinical Scientific Center, Moscow, Russia}

Background: Gastrointestinal stromal tumors (GISTS) are rare clinical entities, representing less than $0.2 \%$ of all gastrointestinal tumors and only $0.04 \%$ of small intestinal malignant neoplasms. GISTs may occur anywhere along gastrointestinal tract, but most commonly arise in the stomach (40-60\%) and jejunum/ileum (25$30 \%$ ). Intussusception and obstruction is uncommon presentation of these lesions because of their tendency to grow in an exraluminal fashion. Methods: Our patient was 29 years old woman attending to Moscow Clinical Scientific Center for colonoscopy with complaints of abdominal cramping pain during a meal for 6 months, weight loss of $5 \mathrm{~kg}$. She was treated at the gastroenterologist without effect. 1 month ago she was hospitalized with symptoms of intestinal obstruction, refused surgery. The patient had also melanoma in remission on target therapy. Results: Examination shown palpable tender mass in the right iliac region. Colonoscopy revealed ileocecal intussusception length $15 \mathrm{~cm}$ with GIST on the top. We have made surgery and found ileocecal intussusception length $28 \mathrm{~cm}$ with stromal tumor size $3 \times 3,5 \mathrm{~cm}$. There was impossible to release ileum and we have performed right hemicolectomy with anastomosis. She was discharged after surgery in satisfactory condition. Conclusion: Adult bowel intussusception is a rare but challenging condition for the surgeon. Preoperative diagnosis is usually missed or delayed because of nonspecific and often subacute symptoms, without the pathognomonic clinical picture associated with intussusception in children. Due to the fact that adult intussusception is often frequently associated with malignant organic lesions, surgical intervention is necessary. Near $90 \%$ cases of intussusception in adults are secondary to a pathologic condition that serves as a lead point, such as carcinomas, polyps, Meckel's diverticulum, colonic diverticulum, strictures or neoplasms, which are usually discovered intraoperatively. GIST must be identified histologically and to be compared with melanoma samples for exluding melanoma progression this patient.

Keywords: Intussusception, Gastrointestinal stromal tumor, lleum

21. Saffron reduces the risk and severity of post-operative peritoneal adhesion in rats

NEDA SARBAZ', Ahmad Mohammadipour'

I Mashhad University of Medical Sciences, Faculty of medicine, Mashhad

Background: Peritoneal adhesions continue to be a major complication of abdominopelvic surgeries. They can cause significant morbidity, including adhesive small bowel obstruction, female infertility and chronic abdominal pain so finding a way to lower the risk of adhesion is necessary. It seems that saffron can reduce the risk of intraabdominal adhesions due to its antiinflammatory properties. The purpose of this study was to determine whether saffron inhibits intra-abdominal adhesions. Material a methods: 45 male rats were administered anesthesia and their abdomens were opened by midline incision. For each rat bowel was abraded with dry gauze and $2.5 \mathrm{cc}$ talc solution $10 \%$ was intrapritoneally injected as the adhesion stimulus. The rats were randomized into three groups including control group(no further treatment), group A (6cc normal saline was injected) and group B $(250 \mathrm{mg} / \mathrm{kg}$ saffron extract was injected). After three weeks, the rats underwent surgery and adhesion grade was evaluated based on the Evans model .The data was then entered into SPSS 22 and analyzed. Significance was accepted when $p<0.05$. Results: There was a significant decrease in incidence rate of adhesion in saffron group $(67 \%)$ than two other groups $(100 \%)$.Data analysis showed that the difference in adhesion grade within saffron group in comparison with control group was statically significant $(\mathrm{P}=0.014)$ and also rats mortality rate in saffron group (6\%)was notably lower than control group $(46 \%)$ and $(p=0.001)$. Conclusion: The use of saffron extract can be effective to prevent adhesion formation after surgery and it also can reduce the adhesion grade in laparotomy surgeries in rats.

Keywords: Saffron ,adhesion ,abdominal surgeries, rat.

\section{Variations of Disease Stage Referring to the Period from} Biopsy to Surgery for Localized Prosate Cancer

JELENA KOVAČEVIĆ', Maša Josipović', Aleksa Jovanović', Boris Kajmaković

School of Medicine, University of Belgrade, Serbia, ${ }^{2}$ Clinic of Urology, Clinical Center of Serbia, Serbia

INTRODUCTION: Prostate cancer is the most common cancer among men(1). Radical prostatectomy is one of the therapeutic modalities. Our aim was to determine whether the duration of waiting period from biopsy to surgery, PSA values, patients' age and size of the prostate correlate with the disease progression and Gleason score (GS), in patients with localized prostate cancer. METHODS: This retrospective study analyzed 102 patients who underwent radical prostatectomy at the Clinic of Urology, Clinical Center of Serbia, from October 2015. to October 2016. The preoperative stage of the disease was verified by sextant TRUS biopsy(1-4). Patients were divided into three groups according to the number of weeks from biopsy to surgery: group I ( 8 ), group I (8-16), group III (216). Patients' age, PSA values and prostate weight were analyzed. The disease progression was defined as an increase in TNM stage, while the GS progression was an increase in the total score or, in case of identical scores, an increase in primary partition(2). RESULTS: There was no statistically significant correlation between the duration of the period from biopsy to 
surgery and the disease progression $(p=0.071) .31 .4 \%$ of patients had an increase in TNM stage, whereas $68.6 \%$ did not. There was no statistically significant difference between these two groups when compared by age $(66.9 \pm 5.6$ vs $65.1 \pm 4.9, p=0.099)$, difference in PSA values ( $9: 46$ vs $10 n g / m l, p=0.986$ ) and the size of the prostate $(42.5$ vs $40 \mathrm{~g} . \mathrm{p}=0.077$ ). The difference between the change of CS and age, PSA values, prostate size and the duration of waiting period from biopsy to surgery was not statistically significant $\quad(p=0.348, \quad p=0.744, \quad p=0.534, \quad p=0.077$, respectively). CONCLUSION: Longer waiting period from biopsy to surgery does not significantly affect the progression of the disease stage as well as values of PSA, age and size of the prostate.

Keywords: localized prostate cancer, Gleason score, PSA, TRUS, radical prostatectomy

23. Vesicoureteral Reflux at the Department of Pediatric Surgery in KBC Rijeka-Experiences

Marija Perić' , Lucija Milolović', Harry Nikolić

7 Faculty of Medicine, University of Rijeka, Rijeka, 51000, Croatia

Introduction: Vesicoureteral reflux (VUR) is characterized by the abnormal flow of urine from the bladder to the upper urinary track. It is the most common congenital urological anomaly affecting $1-2 \%$ of children. Urine normally travels in one direction from the kidneys to the bladder via the ureters, with a flap-valve mechanism at the uretrovesical junction that prevents backflow. Mechanical component of the antireflux mechanism correlates directly with the length of submucosal ureter. Functional component of the ureteral valve mechanism is based on the precise connections of muscle between the ureters and the trigone so the contraction of the bladder pulls the ureters back into the bladder. Primary VUR is a congenital condition caused by the abnormal development and malfunction of the ureterovesical junction. Secondary, it is associated with conditions such as posterior urethral valves, urethral obstruction, or neurogenic bladder dysfunction. Urinary tract infection is the most common factor leading to recognition of VUR. Newborns may have nonspecific symptoms such as poor feeding, irritability and vomiting while older children may report voiding symptoms or abdominal pain. Results: At the Department of Pediatric Surgery in Rijeka since 1966. the treatment of VUR was surgical and the operative techniques were almost entirely extravesical. From 2004. the endoscopic treatment has also been performed. During this time period ( since 1966 to 2012 ), 632 children were treated for VUR. 509 children were operated by conventional methods, whille 123 were treated endoscopic. Success of these conventional methods is up to $97.5 \%$, and the success of endoscopic treatment is $81.9 \%$. Conclusions: If left untreated, Vesicouretral reflux may lead to long-term effects on renal function and overall patient health. Possible complications of this disease are reflux nephropathy, hypertension and kidney failure. These complications may be prevented by a quality and competent treatment of VUR in pediatrics.

Keywords: children, vesicouretral reflux, treatment

Oral session IV:

Surgery, Anaesthesiology

24. Is GDP Correlated With The Frequency of Low Ejection Fraction In Isolated Coronary Surgical Patients?

NADIJA EKINOVIĆ ${ }^{1}$, Damir Kurtagića', Sanko Pandur ${ }^{2}$

7 Faculty of Medicine, University of Sarajevo, Bosnia and Herzegovina, ${ }^{2}$ Clinic of Cardiosurgery, University Clinical Center of Sarajevo, Bosnia and Herzegovina

Background: Reduced ejection fraction (EF) in isolated coronary surgical patients means higher operative risk, and higher possibility of postoperative complications such as postoperative heart failure, disrythmia and prolonged hospitalization. Aim: The aim of this study is to define the number of isolated coronary surgical patients with reduced preoperative EF in University Clinical Center of Sarajevo, and to analyze correlation between EF and GDP of developed and undeveloped countries. Methods: This study is retrospective, descriptive-analytical with statistical processing which was done by using Microsoft Excel. We analyzed 138 coronary cardiac patients who underwent surgery during the period from 01.01.2016. to 30.12.2016. We considered the demographics, EF, number of patients with left main (LM) stenosis, preoperative history of myocardial infarction (MI), percutaneuos coronary intervention $(\mathrm{PCI})$, the number and type of grafts per patient and length of hospitalization. Results: Out of 138 patients who underwent coronary artery bypass surgery, ratio of male to female was $109(79 \%): 29(21 \%)$. The average age of patients was 61.47 years. EF $335 \%$ was found in $24(17.4 \%)$ patients, while $75(54.4 \%)$ had the EF between $36 \%$ and $50 \% .39$ $(28.2 \%)$ patients had $E F>51 \%$. LM stenosis was found in 38 (27.54\%) patients. While $83(60.14 \%)$ patients had $\mathrm{MI}, \mathrm{PCl}$ was performed in only $13(9.42 \%)$ patients. Average of 2.35 grafts was used per patient, with the usage of the mammary artery in 134 (97.1\%) patients. The average number of days that patients spent in hospital was 9.18. Conclusion: Comparing the results that we have, and data obtained from other countries, we came to a conclusion that number of patients who had coronary surgery with reduced ejection fraction in our country is significantly higher. This urges us to find a cost-effective solution that will enable better primary care of acute coronary syndrome.

Keywords: ejection fraction, coronary surgery, acute coronary syndrome, GDP

\section{PEUTZ-JEGHERS SYNDROME: A CASE OF A 21-YEAR OLD FEMALE PRESENTING WITH JEJUNAL INTUSSUSCEPTION} AMINA SOFIĆ ${ }^{1}$, Adi Mulabdić 2

1 Faculty of Medicine, University of Sarajevo, Bosnia and Herzegovina, 2 Department of Abdominal Surgery, University Clinical Center of Sarajevo, Bosnia and Herzegovina

INTRODUCTION: Peutz-Jeghers syndrome (PIS) is an autosomal dominant condition characterized by the presence of gastrointestinal polyps, mucocutaneous pigmentation and a higher risk for developing certain cancers (colorectal, gastric, pancreatic, breast and ovarian cancer). CASE PRESENTATION: We present a case of 21 year old patient admitted as an emergency to the department of abdominal surgery due to severe pain in the abdomen, nausea and vomiting. The patient had clinical and radiological signs of intussusception resulting with ileus. Conservative therapy was not successful; therefore, the same day of admission, the patient underwent exploratory laparotomy under general anesthesia during which a high jejuno-jejunal invagination was discovered with complete obstruction and partial ischemia of the intestinal wall. The obstruction and intussusception was caused by a polypus tumor of the jejunum. The small intestine was resected in two segments and anastomosis was made. Patient made a full recovery and was released from the hospital on the ninth day post operation. Histopathological examination results on biopsy samples were consistent with Peutz-Jeghers syndrome. The patient returned to the hospital to have upper and lower endoscopy and multiple polyps were discovered in the stomach and colon. Biopsy samples were taken and histopathology results confirmed the diagnosis of Peutz-Jeghers syndrome. CONCLUSION: Peutz-Jeghers syndrome is rare and most often first presented as a gastrointestinal intussusception, gastrointestinal bleeding or anemia. Once discovered we must have in mind that the patient has an increased risk of certain tumors in an early age and should act in the best interest of the patient with regular screening examinations of the gastrointestinal tract, breast and gynecological examination for female patients and testicular examination for male patients.

Keywords: Peutz-Jeghers syndrome, intussusception, screening

26. Different levels of potassium over the normal range does not associate with risk of cardiac arrhythmias Valeriia Panafidina ${ }^{1}$

7 First Pavlov State Medical University of Saint-Petersburg, Saint Petersburg, Russia 
Background: Potassium is recognized as a major ion in cardiomyocyte function. Some studies show contradictory results about effect of potassium level on atrial fibrillation in patients after cardiac surgery $[1,2]$. The aim of our study is assessment of significance of different potassium level on the episodes of newonset cardiac arrhythmias in patients with decompensated chronic heart failure Methods: Single-center prospective study. We examined 56 patients which were placed to the ICU with decompensation of chronic heart failure. We evaluate the initial potassium level and if the potassium level was below $3,5 \mathrm{mmol} / \mathrm{l}$ we supplement potassium until normal level. We divided patients into 5 groups: with initial potassium level below $3,5,3,6-4,0,4,1-$ $4,5,4,6-5,0 \mathrm{mmol} / \mathrm{l}$ and higher than $5,0 \mathrm{mmol} / \mathrm{l}$. We evaluate the incidence of arrhythmias during the hospital length of stay. We examined the statistical difference with t-criterion and calculated odds ratio of new-onset arrhythmias. Results: There were no differences between groups in mortality, length of hospital stay or ICU stay. There was no difference in cardiac arrhythmias in patients with normal potassium (more than $3,5 \mathrm{mmol} / \mathrm{l}$ ) or higher potassium. Low potassium level (below the normal range 3,5 $\mathrm{mmol} / \mathrm{l})$ is associated with higher risk of atrial fibrillation and ectopy rhythm (OR 1,8, p,0.001) Conclusion: Potassium level over the normal range does not associate with risk of cardiac arrhythmias.

Keywords: potassium, arrhythmia, chronic heart failure.

27. Neurotoxic Effects of Concentrations of Ropivacaine and Needle Placement in the Sciatic Nerve of Wistar Rats LANA MRDOVIC', Ilvana Hasanbegovic

1 Faculty of Medicine, University of Sarajevo, Bosnia and Herzegovina

Background: Regional anesthesia is a recent method in medicine which negative effects using local anesthetics are not fully discovered. Ropivacaine is often used as an anesthetic in regional anesthesia. The focus of this paper is to test different concentrations of ropivacaine and register its neurotoxicity on sciatic nerve. Our hypothesis is that the neurological injury after intraneural application of ropivacaine will be greater with increasing concentrations of ropivacaine Methods: The sciatic nerves of 50 Wistar rats were exposed bilaterally, under general anesthesia. Total of 100 sciatic nerves were randomized to receive one of four concentrations either of ropivacaine $(0.2 \%, 0.5 \%$, $0.75 \%, 1 \%$ ) of saline intraneurally (R) or perineurally (L) Needles were placed under optic microscopy guidance and opening injection pressure data were acquired using a manometer coupled to a computer. Neurologic examinations were performed at baseline in hours $(1,2,3,4,5,6,24,48,72)$ by blinded assessors. After 72 hours, the rats were sacrificed and the sciatic nerves were histologically examined for quantitative and qualitative evidence of nerve damage. Results: Results showed that inhibition of motor function, nociception function and proprioception were greater with higher concentrations of ropivacaine. Recovery for all three parameters was slower with intraneural needle placement of higher concertation ropivacaine in comparing with perineural needle placement of ropivacaine. Results of quantitative histology showed moderate to severe effects of ropivacaine when the needle placement was intraneural. Conclusion: Needle placement and concertation of ropivacaine influence the nerve damage. Intrafascicularly applied ropivacaine resulted in histologic evidence of nerve damage in all concentrations. Intraneurally injected ropivacaine resulted in increased nerve damage with increased concentrations due to his neurotoxicity. Perineurally injected ropivacaine resulted in concertation dependent duration of blockade without evidence of neurologic injury.

Keywords: ropivacaine, needle placement, neurotoxicity, nerve damage

\section{MRI in Diagnosis of Painful Shoulder}

Đurad Pertović

'Medical faculty Novi Sad, University of Novi Sad, Serbia
Introduction: Shoulder pain is the second most common complaint of patients with diseases of the musculoskeletal system, which occurs in $51 \%$ of patients. Goal: To determine the pathological changes of rotator cuff, glenoid labrum, glenohumeral $(\mathrm{GH})$ and acromioclavicular (AC) joint using MRI in patients with symptoms of painful shoulder in relation to gender and age. Material and methods: The study included 198 patients with pain in the shoulder, and they were divided by gender ( 112 males and 86 females) and age (patients under 30 years, 31 to 60 , and over 61 years). MRI findings were graded with a scale 0 (negative result) or 1 (positive result). We graded, the presence of tear in rotator cuff; the presence of the fluid in $\mathrm{CH}$ joint, subdeltoid and subcoracoid bursa. It was estimated the presence of pathology of glenoid labrum and humeral head as Bankart, Hill-Sachs, SLAP and POLPSA lesion; injury of coracohumeral and glenohumera ligaments; arthrosis $\mathrm{CH}$ and $\mathrm{AC}$ joint; and the existence of subacromial and internal impingement. Results: Statistically significant difference in number of tendon tears in m.SSP by age $(p=0.03)$. Statistically significant difference in number of Bankart and SLAP injuries ( $p<0.05$ ), as well as between number of $A C$ joint arthrosis and subacromial impingement by age $(p=0.02)$. Correlation between number of pathological changes in the distal tendon of m.SSP and number of fluid in subdeltoid bursa, as well as corelation between number of severe arthrosis of $A C$ joint and number of complete tendon tear of m.SSP were statistically significant $(p<0.05)$. Conclusion: Diagnosed fluid in subdeltoid bursa should make suspicion of damage to SSP tendon, while diagnosed severe arthrosis of $A C$ joint should point out on tear of SSP tendon as the cause of pain. Shoulder pain in younger patients is associated with instability of the joint, while in older population is often the result of damage of rotator cuff primarily in SSP.

Keywords: MRI; shoulder; pain; diagnostic imaging

\section{THE IMPORTANCE OF ULTRASOUND VERIFICATION REACTIVELY} ALTERED NECK LYMPH NODES

Dejan Mihajlovic', Bojan Tomic ${ }^{2}$, Aleksandra Ilic

1 Faculty of Medicine, University of Pristina, Kosovska Mitrovica Serbia, 2 Clinic of Radiology, Clinical hospital center Pristina, Gracanica, Serbia

Background: Ultrasound of neck is fast diagnostic procedure to evaluate the essential characteristics of enlarged lymph nodes ,such as aspect/ratio, parenchymal echogenicity, hilar echogenicity and vascularization.(1) Aim: The aim is to establish the parameters for the identification of ehosonografic reactive modified neck lymph nodes. Material and methods: In the period from June to December 2015 at the Clinic of Radiology Clinical Center Pristina, with temporary headquarters in Gracanica, ultrasound were examined 109 patients, of both sexes, aged 12 to 75 years. Patients were sent to the ultrasound examination of the neck after the palpable change observed in the neck. Ultrasound examinations were made on the ultrasounds device "Siemens Acuson $\mathrm{X}_{300}$ ", using multi-frequency probes "VF $10-5$ " frequency range of 5-10 MHz. Echosonographically were followed: aspect / ratio, hilar echogenicity, parenchymal echogenicity, as well as the type of vascularization of the lymph nodes of the neck. Data were analyzed with descriptive statistical methods. Results: of the 109 patients 81 had characteristics of benign enlargement while 28 patients were suspect. In 5 patients had histologically verified malignancy. The frequency ultrasound findings with aspect/ratio $\square$ 0.5 was significantly higher in reactive benign enlargement (89) than histologically verified malignancy (1). Hilar echogenicity and hilar vascularization were significantly more frequent in benign enlargement (104) than histologically verified malignancy (5). The frequency of altered echogenicity parenchyma with reactive benign enlargement is $7 \%$ and at histologically verified malignancy $100 \%$. The altered echogenicity parenchyma was significantly more common among histologically verified malignancy (97) in relation to the reactive benign enlargement (12). Conclusion: The results show that the said ultrasound's parameters has significant a role in the assessment and evaluation of altered neck lymph nodes .

Keywords: lymph node, ultrasound, cancer, neck 
30. Management and fracture prevention of Rotary files during endo-treatment

Omid Panahi', Shabnam Parsaie ${ }^{2}$

' Centro Escolar University, Manila, Philippines, ${ }^{2}$ Urmia University, Urmia, Iran

Background: Breakage of rotary Endodontic files during endotreatment is a recognized complication in endodontics and with simple managements we can prevent and reduce fractured rotary files. Methods: With online search about rotary files in dental journals at specially endodontics journals to retrieve clinical and experimental studies, case reports a review articles by using keywords: management, prevention, fracture and removal. Results: Many cases . rotary files fractures occurred because of canal configurations and incorrect and excessive Conclusion: we can reduce and minimize the risk of rotary files fracture by some important following guidelines: 1.number of uses 2.torque and cyclic fatigue 3 . single use 4.cleaning and sterilization.

Keywords: Breakage, Endodontic, rotary files, management.

31. Effect of chronic immobilisation stress on growth of experimental fibrosarcoma in NMRI mice

PAVLE BANOVIĆ 1 , Đurađ Perović ${ }^{1}$, Dušan Lalošević 7 Medical faculty Novi Sad, University of Novi Sad, Serbia ${ }^{2}$ Department for histology and embryology, Medical faculty Novi Sad, University of Novi Sad, Serbia

Background: Stress is regarded as a significant factor in the etiology of the many diseases. In this study we wanted to examine the impact of chronic immobilization stress on growth of experimental fibrosarcoma in NMRI mice. Methods 20 male NMRI mouses (mus musculus) were randomly divided into 2 groups of 10 mouse - control and experimental. Each mouse was inoculated with $5 \times 106$ freshly trypsinized BHK21 C13 cells. Stress in experimental group was induced by immobilization 2 hours daily for 14 days. After the experiment, tumor masses were removed, measured and histologically analyzed. Statistical analysis included determining differences in tumor expression and tumor volume between groups. Results: Fisher's exact probability test showed a statistically significant difference $(p=0.034)$ in tumor expression between the control and experimental groups. Student t test showed statistically significant difference in tumor volumes between the control and experimental group $(p=0.039)$. In histological analysis of the experimental group material soft tissue tumor was observed, composed of cells that resemble fibroblasts with local infiltrative growth, as well as the absence of immune infiltrate. Histological samples of the control group showed similar tumor tissue morphology,but with the presence of inflammatory, predominantly granulocyte, infiltrate on the edge of the tumor. Conclusion: Chronic immobilization stress, leading to the absence of an effective immune response, stimulates the growth of experimental fibrosarcoma in NMRI mice.

Keywords: stress; immobilisation; fibrosarcoma; mice

Oral session $\mathrm{V}$ :

Neurology, Psychiatry, Family Medicine

32. Diagnostic Possibility of Individual and Combined Use Mini Mental State Examination and Clock Drawing Test in Diagnosing Alzheimer's Disease

PETRANA LAZAREVIĆ' ${ }^{1}$ Natalija Kraković', Monika Klinko', Danilo Marković

School of Medicine University of Belgrade, Belgrade, Serbia

Background: Cognitive impairment is a core and usually first symptom of dementia. Efficient early diagnosis of those with suspected dementia requires quick, meaningful cognitive tests. Mini-mental State Examination (MMSE) and Clock Drawing Test (CDT) are the most used test in the world for the screening of dementia, especially for the initial diagnostic assessment of patients with Alzheimer's disease $(A D)$. Methods: This study included 178 patients. First group were patients with mild cognitive impairment $(\mathrm{MCl})$ and the second group were patients with $A D$. Study compared groups by gender, aged, age at onset of disease, duration of disease and education. It followed results of MMSE, CDT, Addenbrook's test and ADCS-ADL test. Results: Results showed that there is a difference between these groups in final scores of MMSE and CDT, but there is correlation between tests in each group. $15(18.99 \%)$ patients from I group and $69(88.46 \%)$ from II group have score of MMT less than 24 points. Five $(6.33 \%)$ patients from I group and $41(52.56 \%)$ patients from II group have score 1 of CDT. Conclusion: The combination of the MMSE and the CDT was more sensitive and specificity than the MMSE alone, and has nearly the same sensitivity and it is a powerful screening tool for differentiating between patients with $A D$ and patients with $M C l$.

Keywords: Alzheimer's disease, mild cognitive impairment, Clock Drawing Test, Mini-Mental State Examination

33. The frequency and characteristics of Migraine and Tension-type Headache among 6-th year Students of Bukovinian State Medical University (Ukraine)

Vasylieva Nataliya', Shvets Oleksandra'

$\checkmark$ Bukovinian State Medical University, 58000 , Ukraine

Background: WHO has ranked headache among the top 10 disabling conditions worldwide. According to International Classification of Headache Disorders (3-rd edition) the Primary types of Headache divided into 4 groups: Migraine, Tension-type Headache (TTH), Trigeminal Autonomic Cephalgias (TAC), other primary Headache disorders. Headache is the most common presenting complaints of students, predictably due to acclimatization, various physical and psychological stress factors which foreign students are more prone to facing as opposed to general population. Considering this fact, medical students from India as a most numbering group of our Medical University have been the target of this research work. Methods: The crosssectional study was being conducted from September to November 2016 and 85 6-th year students of Bukovinian State Medical University (Chernivtsy, Ukraine) were involved in this investigation. Male-to-female ratio - 1:1.5. The main age of students was $23.2 \pm 2.6$ years, ranging from 22 to 26 years. The diagnosis of different types of headache was made according to the International Headache Society criteria. Results: About $68.2 \%$ of medical students had headache. The prevalence of migraine in the whole cohort was $37.9 \%$. There was a female preponderance. $1 / 5$ of the students had weekly attacks. About $10.3 \%$ students reporting increase in their Migraine headache intensity and frequency with accompanying symptoms such as dizziness, allodynia and neck stiffness. TTH was more common in men $(63.2 \%)$. Conclusion: Trigger factors were identified in $97 \%$ students, predominant of which were poor sleep hygiene, environmental changes, head movements, and mental stress. Only $19 \%$ of students did regular exercise. Twenty-seven percent of students reported self-medication use of analgesics. One-fourth of the students had migraine-associated disability but only $6 \%$ realized that they had migraine.

Keywords: Migraine, headache,students

34.

Evaluation of Cerebrovascular Insult Types and Thei Localization in Women, as a Risk Factor of This Disorder DOLIKA VASOVIĆ ${ }^{1}$, Mirela Vatić ${ }^{1}$, Savić Vujović ${ }^{2}$

'Faculty of Medicine, University of Belgrade, Belgrade, Serbia 2Department of Pharmacology, Clinical Pharmacology and Toxicology, Faculty of Medicine, University of Belgrade, Belgrade, Serbia

Background: The aim of our study was to present and analyze the distribution of cerebrovascular insult types and their localization in women, as a risk factor of this disorder. Methods: In our study we evaluated 990 patients that suffered a cerebrovascular insult and were treated at Special Hospital for Cerebrovascular disorders 
"Saint Sava" in Belgrade. All patients were divided into two groups due to the presence of hemorrhagic or ischemic stroke. Results: Among 990 patients with acute stroke, we found $116(11,7 \%)$ patients with intracebral hemorrhage. Among them 78 were women $(67 \%)$ and $38(33 \%)$ men. Among the intracerebral hemorrhage in women, we found that the most frequent $\mathrm{ICH}$ occurs in the lobar region (56\%), than in the basal ganglia $(37 \%)$, thalamus $(15 \%)$, cerebellum $(5 \%)$, and brainstem (3\%). Among the women who were hospitalized $43(55 \%)$ died, and $35(45 \%)$ were discharged for further rehabilitation or home care. Patients with ischemic stroke $874(88,3 \%)$ were divided into two groups: with hypertension $533(61 \%)$ and without hypertension $341(39 \%)$. Conclusions: It should be stated that women with specific types of cerebrovascular insult as well as their localization are at higher risk for bad prognosis. This study suggests that appropriate diagnostics in appropriate time should be done, because patients with hypertension have more frequent ICH.

Keywords: Cerebrovascular Insult; Women; Hypertension

$$
35 .
$$

\section{Depression}

FATIMA GONDŽo' Ajla Hamidovićn', Momir Šmitran², Srebrenka Bise ${ }^{3}$

1 University of Sarajevo, Faculty of Medicine, Sarajevo, Bosnia and Herzegovina, 2 General Hospital "Prim.dr. Abdulah Nakas" Bosnia and Herzegovina, ${ }^{3}$ Clinic of Psychiatry, University Clinical Center of Sarajevo, Bosnia and Herzegovina

Introduction: Depression (major depressive disorder or clinical depression) is a common but serious mood disorder. It causes severe symptoms that affect how you feel, think, and handle daily activities, such as sleeping, eating or working. In Europe, depression is second most common disorder, in Bosnia and Herzegovina, every tenth adult is depressed. Case description: Female patient, 62 years old, without somatic diseases and negative psychiatric heredity. She became moody, apathetic. Nothing could make her happy. She lost her appetite, so she lost weight. Also, she had stomachache, colic pain and she was sweating. She was thinking that she betrayed her family. Her life has no meaning to her. She lost her interest for life and started to think that would be the best if she was dead. She became suicidal. She wrote a letter and started to suffocate herself with scarf. After unsuccessful attempt of suicide she admits everything to her family. After that she was admitted in Psychiatric hospital Jagomir, in Sarajevo. Her HAMD-17 score was 35 and she had high suicidal risk. Venlafaxin was introduced and dose was increase to $150 \mathrm{mg}$ per day. Improvement was recorded in continuation of treatment, depressive symptoms slowly disappears. Mood and instinctive dynamisms were on rise. Somatic equivalents were retrained. Patient was treated by pharmacotherapeutic and individual supportive psychotherapy. Assessment after three weeks HAMD 17 score was 13. Conclusion: In treatment of Very Severe Depression induction of Velafax shows effective in reduction of apathy and better cognitive and work- social functioning.

Keywords: depression, very serve depression, suicidal risk

The stressors at the natural, social and technical studies students of the Tuzla University

BELMA DEMIROVIĆ ', Ismir Kukić ', Azra Kurtić

' Faculty of Medicine, University of Tuzla, Bosnia and Herzegovina

INTRODUCTION: Stress is a group of unspecific reactions of the human organism to the harmful factors from the working and living environment. AIM: To gain insight into the diversity of stressors and the functionality in the students of natural, social and technical orientations. METHODS AND SURVEY RESPONDENTS: The study used a "Questionnaire on stressors in students of natural, social and technical faculties. "The study included a total of 105 tested survey respondents, which are divided into three groups of 35 students of Medicine, Philosophy, and Electrical engineering. The evaluation of the questionnaire was carried out based on the Likert scale. RESULTS: The most prominent stressful factors in students of Philosophical faculty are: fear of loss of the school year $(60 \%)$, fear of losing health ( $57.41 \%)$, fear of an uncertain future $(34.28 \%)$. For Medical students fear of losing health equals to the fear of loss of the school year ( $51.42 \%)$, and they are also the strongest stressors, followed by the feeling whole daily responsibilities ( $37.14 \%$ ). Among students of Electrical engineering fear of losing health is equal to stress due to constraints of time limits for execution the obligations, and stress related to the inadequate number of employees $(37.41 \%)$. These three stressors are also leading among students of Electrical engineering. The least stressful among the students of Philosophical faculty is lack of communication with colleagues, while Medical and Electrical engineering students find the least stressful conflicts among the colleagues. CONCLUSION: The higher number of the stressors is common for the majority of the survey respondents but in different intensity, which could be easily connected to the current labor market and social conditions, as well as the job they will perform in the future.

Keywords: stress, stressors, students, medicine, electrical engineering, philosophy.

37 .

Use of Evidence based guidelines in rational prescribing of antibiotics for acute respiratory tract infections NDERIME MEMETI', OLIVERA GJEORGJIEVA', Katarina Stavrikj "Medical Faculty Skopje, University "Sts. Cyril and Methodius" Skopje, Macedonia

Background: Evidence based guidelines for acute respiratory tract infections are well established. In Macedonia are introduced in $2007.80 \%$ of RTI's are viral infections and according the guidelines should not be prescribed antibiotics. Materials and methods: A cross sectional study, based on a representative sample of GPS was conducted. 107 general practitioners were invited to participate and 86 doctors have finished the study (80\%).All patients with RTI's were involved in the survey. Results: A total number of 9154 patients, with age from 6 months to 94 years, with RTI's were reported in a period of 4 weeks in November 2014 Most common diagnosis was cold $(24.9 \%)$ acute tonsillitis $(20 \%)$, acute bronchitis (19\%) and acute pharyngitis (14.2\%).5329 patients with RTI's $(58.2 \%)$ were treated with antibiotics and most prescribed antibiotic for ARTI was amoxicillin + clavulonic acid $(18.7 \%)$, amoxicillin (11.4\%) and cephalosporins (8.4\%). Although $80 \%$ of those RTI's are viral, $12.6 \%$ of patients with cold were treated with antibiotics, $80 \%$ with acute tonsilopharyngitis and $68.2 \%$ with acute bronchitis. In patients with cold most dominant symptom was rhinorrhea and cough, and antibiotics with broad spectrum were prescribed: amoxicillin (37.5\%) amoxicillin+ clavulonic acid (25.4\%) as well as cephalosporin (25\%). 1571 patients $(88.8 \%)$ of patients with acute pharyngitis were treated with antibiotics although 832 patients (87.3\%) with Centor score $>=$ 2 were treated unnecessary with antibiotics. The most prescribing drug was amoxicillin-clavulonate (622 patients), while only $47 \%$ of patients received a recommended antibiotic (amoxicillin, penicillin V, cephalexin) according the guideline. Conclusion: This study showed prescription of many broad spectrum antibiotics for acute RTIs. Although there are guidelines, the compliance is very low. The GPS should work according to the given guidelines and hesitate the pressure from the patients. That is the only way we would be able to decelerate the evolution of antibiotics resistance.

Keywords: antibiotics, RTI's, guidelines

38.

PHYSICAL, CHEMICAL AND MICROBIOLOGICAL QUALITY OF WATER SAMPLES IN KARAMAN PROVINCE

Sami Karagöz ${ }^{1}$, Mehmet Fatih Aydin ${ }^{2}$, Süleyman Gökmen ${ }^{3}$ 1 Public Health Laboratory, Karaman, 70200, Turkey, 2 Faculty of Health Sciences, Karamanoglu Mehmetbey University, Karaman, 70100, Turkey, ${ }^{3}$ Technical Vocational School of Higher Education, Karamanoglu Mehmetbey University, Karaman, 70100, Turkey

Background: Water quality is important to protect public health and also for the environment. It was aimed with this study to determine the physical, chemical and microbiological quality of water samples in rural and urban areas of Karaman province. 
Methods: Natural spring, city and pool water samples were collected into the sterile bottles of 250 milliliters and non-sterile bottles of 1500 milliliters for microbiological and physico-chemical analysis respectively during January to July 2016. Some physicochemical parameters (taste, color, odour, turbidity, total hardness, transparency, pH, ammonium, chlorides, phosphate and nitrates) were analyzed. Chemical analyses were performed by using commercial kits. Before bacterial inoculation water samples were filtered by the membrane filtration system and analyzed in terms of coliform bacteria. Results: Twenty eight of 71 drinking water samples for special request, 156 of 301 drinking water samples for control, 3 of 24 water samples for pool water and none of 18 drinking water for market surveillance and inspection were found unsafe in terms of microbiological analysis. Nine of 52 drinking water samples for special request, none of 267 drinking water samples for control and 19 of 25 water samples for pool water were found unsafe in terms of physico-chemical analysis. Conclusion: It was detected that some water samples in Karaman province were found unsafe for microbiological and for some physico-chemical criteria. It was evaluated that the reason of unsafe pool water results was related to bacterial contamination of pools and artesian well water source.

Keywords: Water safety, Karaman, physicochemical and microbiological quality

Oral session VI:

Internal Medicine

39. Changes in the Morphology and Function of the Left Ventricle in Patients Undergoing Hemodialysis

MONIKA KLINKO', Danilo Marković', Petrana Lazarević

'Faculty of Medicine, University of Belgrade, Belgrade, Serbia

Background: Cardiovascular diseases are the leading cause of morbidity and mortality in patients treated with hemodialysis. Heart failure is present in $40 \%$ of patients at the beginning of treatment. Left ventricular (LV) hypertrophy is associated with diastolic dysfunction and for its assessment, in clinical practice Doppler and Tissue Doppler echocardiography are used. Based on the dimensions of LV it is possible to assess ventricular function, mass and volume and to determine LV mass index (LVMI). In this study, we wanted to show the morphological and functional characteristics of the left ventricle in patients with end-stage chronic kidney disease and structural and functional changes after 9 months of treatment with hemodialysis. Methods: A prospective study of patients with terminal kidney disease on chronic hemodialysis program at Clinical Hospital Center "Zvezdara" from 21 February 2012 to 26 June 2014. Doppler ehokardiographic measurements and indexes are made in two time intervals of nine months. Results: Dimensions of interventricular septum and LVMI were significantly reduced, the average maximum velocity of late ventricular filling (A) as well as the E/A. Reducing the rate of atrial filling phase of LV is interpreted with better diastolic function of LV. There has also been an improvement of global systolic function, based on changes of EF. The parameters of diastolic LV function such as E, E/A and E/e 'are closely connected with the change of structural LV parameters. Changes of the global EF LK are significantly affected by changes in ESD, ESV, and LVMI after nine months of hemodialysis treatment. Conclusion: Doppler echocardiography has an important role in the assessment of LV function in patients undergoing chronic hemodialysis. A significant contribution to the treatment is reducing the hypertrophy of the walls, LVMI and improving parameters of systolic and diastolic LV function.

Keywords: Hemodialysis; Left ventricle; Echocardiography

40.

Clinical Presentation of Double Diabetes

NAZLI BEGUM OZTURK', Kubilay Karsidag

7 Istanbul University, Istanbul Faculty of Medicine, Istanbul,

Turkey, Istanbul University, Istanbul Faculty of Medicine, Department of Internal Medicine, Division of Endocrinologyemetabolism, Istanbul, Turkey
Introduction: Double diabetes is a combination of type 1 diabetes(T1DM) with features of insulin resistance and type 2 diabetes(T2DM). Patients with T1DM who had a family history of T2DM were more likely to be overweight and rarely achieved adequate glycaemic control'. We present a young-adult with double diabetes. Case Report: A 32-year-old man was admitted to hospital because of severe polyphagia and polydipsia for 5 years. His blood sugar was $280 \mathrm{mg} / \mathrm{dl}, \mathrm{BMl}$ was 32,58 and he was diagnosed with T2DM. Management of nutrition and sulfonylurea were implemented for the treatment. His blood sugar regressed to 110-120 mg/dl and he didn't agree with the treatment henceforth. During the following months, he had a febrile illness and his blood sugar was measured as $276 \mathrm{mg} / \mathrm{dl}$, HbA1C \%10,5, C-peptid 1,3 $\mathrm{ng} / \mathrm{ml}$, cholesterol $323 \mathrm{mg} / \mathrm{dl}$. ICA and anti-GAD was negative and acetonuria was seen. His mother and aunt were diagnosed with T2DM. His medications included metformin, statin, acetylsalicylic acid and combination insulin. The patient's phenotypic features, family history, strong beta cells reserve and non-founded autoimmune indicator looked like T2DM, but ketonuria, onset of the disease and process of the patient considered him as a T1DM. on the current evaluation, the patient diagnosed with an autoimmune disease; ulcerative colisitis. ICA was negative, antiGAD $494 \mathrm{U} / \mathrm{ml}$, C-peptid $0,29 \mathrm{ng} / \mathrm{ml}$. After seeing anti-GAD autoantibodies, he was diagnosed with double diabetes. Conclusion: Double diabetes has no exact diagnostic criteria. comparisons and contrasts between T1DM and T2DM are highlighted in terms of hepatic fat, fat partitioning, lipid profile and how these may differ between type 1 diabetic patients with and without double diabetes ${ }^{2}$. It is important to determine who has double diabetes since that will help to dictate which diagnostic and therapeutic approaches should be taken ${ }^{3}$.

Keywords: diabetes, double diabetes, insulin resistance, obesity

\section{Correlation Between DHEAS And Tumor Size In} Patients With Adrenal Incidentaloma

MARKOVIĆ DANILO 1, Jadžić Jelena 1, Lazarević Petrana ', Klinko Monika ', Vatić Mirela', Marina Ljiljana ${ }^{2}$

1 School of Medicine, University of Belgrade, Belgrade, 11000, Serbia, ${ }^{2}$ Clinic for endocrinology. Clinical center of Serbia, Belgrade, 11000, Serbia

Background: Adrenal incidentaloma ( $\mathrm{Al})$ is an adrenal enlargement in patient without any previous doubt on hormonal hypersecretion, accidentally discovered by imaging techniques. Al considering the hormonal activity can be functional, nonfunctional (NAI), or subclinical hypercorticism (SH). So far, there is no uniform diagnostic criteria for SH. Recent studies have considered the use of dehydroepiandrosterone-sulfate (DHEAS) as a diagnostic criterion. DHEAS relation to the tumor size is still uncertain. It is important to determine it, because the tumor size is the diagnostic criterion directly correlated with hormonal hypersecretion, and it is an important factor in deciding on surgical treatment. Methods: We retrospectively examined 139 patients. There were 73 patients with NAI, mean age $60.23 \pm 11.6$ years, BMI $28.71 \pm 5.56 \mathrm{~kg} / \mathrm{m} 2$ and DHEAS level of $1.66 \pm 1.27$ $\mathrm{mmol} / \mathrm{l}$. SH was diagnosed if the patient meets at least 2 of 3 criteria: cortisol. $83 \mathrm{nmol} / \mathrm{l}$ in overnight $1 \mathrm{mg}$ dexamethasone suppression test (DST), suppressed ACTH , $14 \mathrm{pg} / \mathrm{ml}$, and midnight cortisol . $150 \mathrm{nmol} / \mathrm{l}$. Out of all, 66 patients had SH, age $59.45 \pm$ 7.95 years, BMI $28.53 \pm 4.79 \mathrm{~kg} / \mathrm{m} 2$ and DHEAS $0.70 \pm 0.53 \mathrm{mmol} / \mathrm{l}$. Tumor size was measured by CT. Pearson correlation and Student's t-test were used in statistical analysis. Results: There is no statistically significant differences between groups in gender, age and BMI ( $p>0.05)$. SH has significantly lower levels of DHEAS $(p<0.001)$ and larger tumor size $(p<0.001)$, compared to the NAI. DHEAS is significantly correlated with the tumor size $(r=-0,195, p=$ $0.028)$, ACTH ( $r=0.254, p=0.004)$ and cortisol in DST ( $r=-0.254, p=$ $0.004)$. Conclusion: The results of our study show the significantly lower level of DHEAS in patients with SH, directly correlated with the tumor size, ACTH and cortisol levels in DST. This finding supports the use of DHEAS as a potential diagnostic criterion for $\mathrm{SH}$.

Keywords: DHEAS, adrenal incidentaloma, tumor size 
Correlation Between Lipid Parameters and Mean Platelet Volume in Adult Patients with Rheumatoid Arthritis MALIK GALIIAŠEVIĆ', Amela Dervišević2 Nesina Avdagić 2 ' Faculty of Medicine, University of Sarajevo, Bosnia and Herzegovina, 2Department of Human Physiology, Faculty of Medicine, University of Sarajevo, Bosnia and Herzegovina

Background: Rheumatoid arthritis (RA) is a common chronic autoimmune disease associated with increased risk of cardiovascular morbidity and mortality. Dyslipidemia is one of the major risk factors for coronary artery disease (CAD). Increased mean platelet volume (MPV), which is a marker for platelet activation, is a central process in the pathophysiology of CAD. The aim of the study was to get a lipid profile in adult patients with RA and to evaluate the correlation of lipid parameters with MPV. Methods: This cross-sectional study included 60 ( 56 females and 4 males) RA patients and 28 (23 females and 5 males) age- and gender-matched apparently healthy individuals. All patients fulfilled the American College of Rheumatology $(A C R)$ criteria for RA. A standard biochemical analysis was performed to assess serum concentrations of total cholesterol (TC), triglycerides (TCC), high-density lipoproteins (HDL-C), low-density lipoproteins (LDL-C) and mean platelet volume (MPV) of all subjects. Appropriate statistical tools were used to calculate statistical difference using SPSS for Windows Version 13.0 Results: The patients median age was $54.7 \pm 11.8$ years. There were no statistically significant differences regarding age $(\mathrm{p}=0.06)$ and gender $(0.22)$ in the RA and control groups. Significantly higher levels of serum TCC $(p=0.006)$, HDL-C $(p=0.032)$ and LDL-C $(p=0.002)$ were observed in RA patients as compared to healthy controls. However, the two groups did not show any significant difference in the level of serum TC $(p=0.131)$. The MPV value was statistically significantly higher in RA patients compare to healthy subjects. However, there were no statistically significant correlations between lipid parameters and MPV in RA group. Conclusion: We determined that RA patients had a high MPV value and altered lipid profile, and these would be expected to change the pattern of cardiovascular events in RA.

Keywords: Rheumatoid arthritis, Mean platelet volume (MPV), Lipid parameters

\section{Cross-sectional Study of Dyspeptic Symptoms in Patients} with Metabolic

ALEKSA JOVANOVIĆ', Maša Josipović', Jelena Kovačević' Tomica Milosavljević

'School of Medicine, University of Belgrade, Belgrade, Serbia, ${ }^{2}$ Clinic for Gastroenterology and Hepatology of Clinical Center of Serbia school of Medicine, University of Belgrade, Serbia

Background: Dyspepsia is a clinical syndrome consisting of various symptoms in the upper abdomen such as epigastric pain, bloating, heartburn, nausea, vomiting(1). Metabolic syndrome is consisted of insulin resistance, hypertension, visceral adiposity, etc. which are risk factors for cardiovascular disease and type II diabetes(2). Our aim was to estimate the prevalence of dyspeptic symptoms on a sample of patients with metabolic syndrome. Methods: This multicenter cross-sectional study included 126 patients with metabolic syndrome from several cardiology clinics in Serbia. Data was collected using an anonymous questionnaire and was analyzed using descriptive statistics and $\chi^{2}$ test. Results: $67.29 \%$ of the patients were male and $32.71 \%$ female. Mean age was $59.6 \pm 10.2$ years. $58.73 \%$ of patients had dyspeptic symptoms, $80 \%$ of women compared to $48.6 \%$ of men, the difference being statistically significant $\left(X^{2}=9.584, p=0.002\right) .34 .2 \%$ of patients with symptoms had a diagnosed stomach condition, the most common being gastritis $(68.8 \%)$, gastric ulcer $(18.8 \%)$, and gastroesophageal reflux disease (12.5\%). In patients with dyspeptic symptoms, reflux was most common ( $45.8 \%)$, followed by bloating $(36.1 \%)$, burping (20.8\%), nausea and epigastric pain (16.7\%). $30 \%$ of patients had symptoms few times a month, $20 \%$ few times a year, $15.7 \%$ once in a month, $14.3 \%$ couple of times a week. $27.8 \%$ of patients believe the symptoms are provoked by use of medication, $26.4 \%$ think emotional stress is the trigger, $26.4 \%$ don't know, $12.5 \%$ think that coffee, alcohol or fast food are the cause. Conclusion: This study showed a higher prevalence of dyspeptic symptoms in the population of patients with metabolic syndrome, than what the literature data shows for general population (20$40 \%)(3,4)$. Dyspepsia is associated with a reduction in quality of life and seeing how high the prevalence is in patients with metabolic syndrome, they should be considered an important group for diagnosing and treating the causes of dyspeptic symptoms.

Keywords: Dyspepsia; Metabolic Syndrome; Prevalence

44. Increased FSH and LH are associated with bone loss during early postmenopausal period

Amina Blekić', Amina Valjevac

7 Faculty of Medicine, University of Sarajevo, Bosnia and Herzegovina, 2Department of Human Physiology, Faculty of Medicine, University of Sarajevo, Bosnia and Herzegovina

Background: Pathophysiology of postmenopausal osteoporosis has been considered due to deficiency of estrogen. However, it has been reported that the rate of bone mass loss during perimenopause is greater than that in postmenopause, which might be related to the increase in FSH and/or $\operatorname{LH}(1,2)$. Therefore, the aim of the study was to evaluate the association between gonadotropins and bone mineral density in postemenopausal females. Methods: The study included 180 postmenopausal females who were divided into two groups based on the bone mineral density (BMD) values; osteoporosis group ( $0 \mathrm{G}, \mathrm{N}=90)$ and the control group $(C G, N=90)$. BMD was determined by dual $X$-ray absorptiometry - DXA). Gonadal steroids were measured by competitive immunoassay. Results: Serum FSH and LH levels were significantly higher in OC compared to CG $(54.3 \pm 2.17$ vs. $43.0 \pm 2.38$ IU/L; $\quad \mathrm{p}<0,001$ and $28.9 \pm 1.36$ vs. $23.67 \pm 1.46$ IU/L; $\quad \mathrm{p}=0.009$ respectively). In postmenopausal females, serum FSH and LH levels were negatively associated with total hip BMD $(r=-0.23$ and $r=-0.22 ; p<0.01)$ and total lumbar spine BMD $(r=-0.27$ and $r=-0.21$, $p<0.01)$. When the females were stratified by duration of menopause, negative association between FSH, LH and total hip and total lumbar spine BMD was only observed in group of females during the first decade since menopause. Conclusion: Increase in FSH and LH during menopause might lead to decreased bone mineral density especially during the first decade since menopause, suggesting additional therapeutic measures for decreasing gonadotropins might be a helpful strategy to prevent bone loss.

Keywords: postmenopausal females, bone mineral density, follicle stimulating hormone, luteinizing hormone, osteoporosis.

45. Lipid profile in patients with diabetes mellitus type 2 with and without metabolic syndrome

Meis Sammak ${ }^{1}$, Selma Kulenovic ${ }^{1}$, Hajrudin Musić ${ }^{\text {, Amela }}$ Dervišević ${ }^{2}$

- Faculty of Medicine, University of Sarajevo, Bosnia and Herzegovina, 2 Department of Human Physiology, Faculty of Medicine, University of Sarajevo, Bosnia and Herzegovina

BACKGROUND : Metabolic syndrome is a cluster of metabolic disorders that raises risk for heart disease, diabetes and stroke. It is presented with at least 3 of the 5 following conditions abdominal obesity, triglyceride level of $150 \mathrm{mg} / \mathrm{dL}, \mathrm{HDL}$ cholesterol of less than $40 \mathrm{mg} / \mathrm{dL}$ in men or less than $50 \mathrm{mg} / \mathrm{dL}$ in women, systolic blood pressure $130 / 85$ or greater and fasting glucose of $100 \mathrm{mg} / \mathrm{dL}$ or greater. The presence of metabolic syndrome is associated with a higher prevalence of CVD in patients with type 2 diabetes. For that reason this study focused on comparing values of lipid profil in patients with DM2 with and without metabolic syndrom. MATERIALS AND METHODS: This retrospecive study included 186 patients with diabetes mellitus type 2 hospitalised in 2014 at the Clinic of Endocrinology, Diabetes and Metabolic Diseases, KCU Sarajevo, who had completed data from the history of illness. Patients were divided into 2 groups based on the criteria of NCEP/ ATP III for the diagnosis of metabolic syndrome: patients with diabetes mellitus type 2 and metablic syndrome (MS-DM2) and patients with diabetes mellitus type 2 without 
metabolic syndrome (DM2-NMS). RESULTS: The incidence of metabolic syndrome with type 2 diabetes was $58,6 \%$, and $41,4 \%$ of the patients didn't have metabolic syndrome. The median value of the concentration of triglycerides in serum was higher in the group DM2-MS and that was statistically significant. Patients with metabolic syndrome had lower concentration of HDL cholesterole in serum than patients without metabolic syndrome. Median value of the concentration of total cholesterole and LDL cholesterole was higher in patients with metabolic syndrome but that was not statistically significant. CONCLUSION: The metabolic syndrome is significantly presented in patients with diabetes mellitus type 2 . There are differences in lipid status in 2 groups of the patients, especially in concentration HDL cholesterole and triglycerides.

Keywords: metabolic syndrome, diabetes mellitus type2, lipid status

46. Nonalcoholic Fatty Liver and The Severity of Acute Pancreatitis

TAJANA STEVANOVIĆ' Ivana Mikolasević3

'Faculty of Medicine, Rijeka, Croatia, 'Department of Nephrology, Dialysis and Kidney Transplantation, UHC Rijeka, Croatia, 3Department of Gastroenterology, UHC Rijeka, Croatia

AIM: to explore the effect of nonalcoholic fatty liver (NAFL) as a hepatic manifestation of metabolic syndrome on the severity of acute pancreatitis. Our hypothesis was that patients with nonalcoholic fatty liver would have a more severe form of disease. PATIENTS AND METHODS: We retrospectively analyzed 822 patients hospitalized with acute pancreatitis. We diagnosed acute pancreatitis and determined its severity according the revised Atlanta classification criteria from 2012. We assessed nonalcoholic fatty liver with computed tomography. RESULTS: There were 198 $(24.1 \%)$ patients out of 822 analyzed who had nonalcoholic fatty liver. Compared to patients without nonalcoholic fatty liver, patients with nonalcoholic fatty liver had significantly higher incidence of all components of metabolic syndrome. Patients with nonalcoholic fatty liver had statistically higher incidence of moderately severe $(35.4 \%$ vs. $14.6 \% ; p=0.02)$ and severe acute pancreatitis $(20.7 \%$ vs. $9.6 \% ; p<0.001)$ compared to patients without nonalcoholic fatty liver. At the admission patients with nonalcoholic fatty liver had higher values of C-reactive protein, higher APACHE II score at admission and significantly higher incidence of organ failure and local complications as well as higher values of computed tomography severity index compared to patients without nonalcoholic fatty liver. Compared to patients without nonalcoholic fatty liver, patients with nonalcoholic fatty liver had a higher death rate, however not statistically significant ( $5.6 \%$ vs. $4.3 \%$; $p=N S$ ). CONCLUSION: Presence of nonalcoholic fatty liver at admission can indicate a higher risk for developing more severe forms of acute pancreatitis and could be used as an additional prognostic tool.

Keywords: nonalcoholic fatty liver, acute pancreatitis, severity, prognosis.

\section{Adiponectin is associated with increased bone turnover in} postmenopausal females

Vanis Nedim ', Horozić Džan ', Sokolović Emir ', Valjevac Amina, MD, PhD '

Faculty of Medicine, Sarajevo, 71000, Bosnia and Herzegovina

Background: Adiponectin has been shown to increase osteoblast proliferation and differentiation while inhibiting osteoclastogenesis in vitro. However, the effects of adiponectin on bone metabolism are not univocal among different experimental systems and between in vivo and in vitro studies. So the aim of the study was to investigate the relationship between adiponectin, bone mineral density and bone remodeling marker in postmenopausal females. Methods: The study included apparently healthy 180 postmenopausal females in whom we measured bone mineral density (BMD) and bone mineral content (BMC) by dual $\mathrm{X}$-ray absorptiometry. The urine NTx levels were determined by chemiluminescent method on Vitros ECiQ Immunodiagnostic System. Serum levels of adiponectin were determined with Radioimmunoassay (RIA) and serum osteocalcin was determined by electrochemiluminescence immunoassay. Results: Mean serum adiponectin level in the study sample of 180 postmenopausal females was $11.56 \pm 0.37 \mu \mathrm{g} / \mathrm{mL}$. Mean serum adiponectin level in obese subjects $(8,86 \pm 4,5 \mu \mathrm{g} / \mathrm{mL})$ was significantly lower compared to overweight $(12,1 \pm 5,4 \mu \mathrm{g} / \mathrm{mL})$ and normal weight females $(13,3 \pm 4,4 \mu \mathrm{g} / \mathrm{mL})$. Serum adiponectin level was negatively associated with total hip BMD $(r=-0,32 ; p<0,01)$ and $B M C(r=-0,28$; $p<0,01)$ and lumbar spine BMD $(r=-0,19 ; p<0,01)$ and $B M C(r=-0,20$; $p<0,01)$. Serum adiponectin level was significantly positively associated with both bone formation marker osteocalcin $(r=0,213$; $p<0,01)$ and bone resorption marker urinary NTX levels $(r=0,16$; $p<0,05)$ Conclusion: In our study, obese females have had significantly lower adiponectin levels compared to overweight and normal weight patients. Also, adiponectin was negatively associated with total hip and lumbar spine BMD and BMC. Bone formation marker (osteocalcin) and bone resorption marker (urinary NTX levels) have been positively associated with adiponectin serum level. Therefore, adiponectin may have influence on faster bone turnover in postmenopausal females. As a conclusion, lower adiponectin level decreases risk of osteoporosis in obese postmenopausal females.

Keywords: Adiponectin, postmenopausal females, bone mineral density, bone turnover

\section{Oral session VII:}

\section{Internal Medicine}

48. Prevalence of electrocardiographic signs of the left ventricular hypertrophy in patients with different stages of arterial hypertension

Mirza Pračić1*, Dino Pračić1, Nermina Babićn ${ }^{2}$ Nesina Avdagić ${ }^{2}$

1 Faculty of Medicine, University of Sarajevo, Sarajevo, 71000 Bosnia and Herzegovina, 2 Department of Human Physiology, Faculty of Medicine, University of Sarajevo, Sarajevo, 71000 Bosnia and Herzegovina

Background: Arterial hypertension is a major risk factor in cardiovascular diseases. Electrocardiography plays an important role in the early detection of complications of hypertension and in the assessment of overall cardiovascular risk. Aim: To determine if there are significant differences in the prevalence of electrocardiographic left ventricular hypertrophy signs, estimated by Sokolow-Lyon (SL), RaVL, and the Cornell voltage criteria between patients with hypertension grade I, II and III, and between newly diagnosed patients, patients taking the therapy 110 years and more than 10 years. Methods: The study included 107 patients of both sexes, who were diagnostically and therapeutically treated at the Clinic for cardiology, KCUS, in the period from the beginning of 2014. until the end of April 2015. Patients were divided into groups HTA I $(n=37)$, HTA II $(n=37)$, HTA III $(n=33)$, and based on the duration of the disease: newly discovered, 1-10 years and $>10$ years. Obtained data were analyzed by descriptive statisticalanalysis. Results: Statistically significant differences are shown in the prevalence of subjects with/without filled S-L criteria for left ventricular hypertrophy between groups I and HTA HTA III $(p=0.018)$, prevalence of subjects with / without meeting Cornell criteria between HTA group I and II hypertension $(p=0.019)$ and between groups HTA I and HTA III $(p=0.007)$, and differences in patients with/without filled S-L criteria, a group of hypertensive patients with disease duration between 1-10 years and groups of hypertensive patients with disease duration of 10 or more years $(p=0.005)$. There were no statistically significant differences in the prevalence of subjects with/without filled RaVL criteria for left ventricular hypertrophy between the groups. Conclusion: Higher the grade of hypertension, higher the percentual representation of patients who meet the $S-L$ and Cornell voltage criteria for hypertrophy of the left ventricle. The results also suggest that increasing the duration of the disease 
increases the percentage of patients who meet the S-L criteria for left ventricular hypertrophy is greater.

Keywords: arterial hypertension, electrocardiography, left ventricular hipertrophy

49.

The Role of Infused $\mathrm{CD}_{34+}$ Cells Dose on Prognosis of
Patients with Hodgkin's Lymphoma after Autologous Stem
Cell Transplantation
Nikola Spremić
' Faculty of Medicine, University of Belgrade, Belgrade, 11000 ,
Serbia

Background: Early lymphocyte, neutrophil, platelet recovery, and infused dose of $\mathrm{CD} 34+$ cells, have been associated with clinical outcome of patients with Hodgkin's lymphoma (HL). Methods: This retrospective study included 87 patients with $\mathrm{HL}$ (median age 31 years, range 20-52 years; 48 males/39 females) who underwent ASCT between June 2005 and June 2016. At diagnosis, advanced Ann Arbor stage had $63.2 \%$ of patients, B symptoms $85.1 \%$, bulky disease $49.4 \%$ and high IPS (International Prognostic score) had $50.7 \%$. Results: The average of transplanted CD34+ cells in $\mathrm{HL}$ patients was $7.20 \times 106 / \mathrm{kg}$ (range $2-25 \times 106 / \mathrm{kg}$ ) and favourable treatment response (partial/complete remission) achieved $82.8 \%$ HL patients, of whom $18.3 \%$ had infused $<5 \times 106 / \mathrm{kg}$ CD $34+$ cells. Median time to recovery of ALC500 was 16 days (range 9-31 days), recovery of ANC500 was 12 (range 6-26 days), and PLT20 was 12 (range 5-44 days). Median follow up of patients with HL was 66 months, while after ASCT, median event free survival (EFS) was 21 months, and overall survival (OS) was 39 months. Achieving partial or complete remission after ASCT strongly influenced both EFS and OS after ASCT $(p<0.0001)$. In those patients, OS and EFS were influenced by infused $C D 34+$ cell dose $(<5 \times 106 / \mathrm{kg}$ vs. $.5 \times 106 / \mathrm{kg})$, recovery of $\mathrm{ALC} 500$ by Day +20 , and PLT by Day +13 . Multivariate analysis showed that infused $\mathrm{CD}_{34}+$ cell dose was the most important parameter that influenced OS and EFS after ASCT ( $p<0.05)$. Conclusion: The number of infused CD34+ cells, as an independent factor, may affect OS and EFS after ASCT of patients with HL.

Keywords: Hodgkin's lymphoma, autologous stem cell transplantation, treatment response, prognosis

The use of High Resolution Melting (HRM) in detection of new mutations in chronic lymphocytic leukemia (CLL) patients.

Jacek Zawislak', Maciej Putowski', Marta Podgórniak', Marta Piróg', Joanna Knap'

Medical University of Lublin, Lublin, 20-059, Poland

Background: $\mathrm{SF}_{3} \mathrm{~B}_{1}$ mutations are genomic alterations found in chronic lymphocytic leukaemia cells. The mutation is more frequently detected in cases with unfavorable clinical course, but it is not considered a prognostic marker yet. Currently, the SF3B 1 detection is based on Sanger sequencing, which is a timeconsuming and expensive procedure. It is done by nucleobases searching performed for each patient. Consequently, a new reliable screening method is highly required. HRM analysis is based on small variations of melting temperature points between amplicons harboring the mutations and the wild types. It was performed in real-time with the results presented on the melting curves. During the study, the HRM analysis was performed as a screening for $\mathrm{SF}_{3} \mathrm{~B}_{1}$ mutations for a representative population of the Central Europe for the first time. Methods: Peripheral blood mononuclear cells were taken from samples of 364 CLL patients. Following $\mathrm{SF}_{3} \mathrm{~B}_{1}$ mutations were evaluated: K700, E622/R625 and H662/K666 (exons 14 and 15) by using HRM detection and the results were validated by Sanger sequencing. Results: $\mathrm{SF}_{3} \mathrm{~B}_{1}$ mutations were observed in $17 / 364$ patients $(4,7 \%)$ : 9 of them were presented in exon 14 while 8 occurred in exon 15. The presence of $\mathrm{SF} 3 \mathrm{~B} 1$ mutation was distinguished in 17 of 364 samples (which represents $4,7 \%$ ). K700 was the most frequent type of $\mathrm{SF}_{3} \mathrm{~B} 1$ mutation: $47 \%$ of all mutations. As a result, the HRM analysis was considered a simple and quick method of recognizing
$\mathrm{SF}_{3} \mathrm{~B} 1$ which can be executed in even 300 samples at once. Unfortunately, the sensitivity to the reaction conditions causes some false positive results. Conclusion: All things considered, HRM analysis proves to be decent screening technique of detecting $\mathrm{SF}_{3} \mathrm{~B} 1$ mutations. It can significantly improve laboratory work. Since $\mathrm{SF}_{3} \mathrm{~B}_{1}$ mutations can be classified as negative prognostic factors in CLL, HRM seems to be a considerable step forward in forming improved classification of the disease.

Keywords: High Resolution Melting (HRM) analysis; detection of $\mathrm{SF}_{3 \mathrm{~B} 1} 1$ mutations; chronic lymphocytic leukemia (CLL) widespreading of $\mathrm{SF} 3 \mathrm{~B} 1$ marking.

51. Time to first treatment with regard to currently available prognostic factors and novel mutations in CLL JOANNA KNAP_1, MARTA PIRÓG ', MARTA PODGÓRNIAK ', MACIEJ PUTOWSKI ', JACEK ZAWIŚLAK

${ }^{7}$ Medical University of Lublin, 20-001, Lublin, Poland

Background: Chronic lymphocytic leukemia (CLL) is the most common leukemia in adults in Western hemisphere. It is characterized by various clinical outcome, including cases requiring treatment at time of diagnosis, delayed therapy in symptomatic disease, or indolent disease never requiring treatment. The prognosis depends on the stage of the disease, as well as the presence of estimated prognostic factors. The indicator of progression in CLL is time to first treatment (TTFT), measured as the time from diagnosis to the beginning of therapy. Methods: We isolated DNA from peripheral blood mononuclear cells of 202 patients. Then we performed ARMS-PCR to investigate $\mathrm{NOTCH}_{1}$ C.7544 7545delCT in PEST domain and MYD88 L265P mutation. Screening for $\mathrm{SF}_{3} \mathrm{~B} 1$ mutations K700, E622/R625 and H662/K666 (exons 14 and 15) were performed using High Resolution Melting Analysis and the results were confirmed by Sanger sequencing. We also used Sanger sequencing for ICHV gene mutation status assessment. Clinically, we assessed the prognostic significance in terms of TTFT. The data were analyzed using GraphPad Prism software. Results: The assessment of median TTFT revealed the significant differences between patients from various prognostic groups. Patients with unmutated IGHV gene status were characterized by significantly shorter TTFT than patients harbouring the mutation ( $p<0.0001)$. The similar correlation occurs in patients with ZAP-70 positive $(p=0.04)$ and $C D 38$ positive $(p=0.0003)$. There were no significant differences in patients with mutated and unmutated $\mathrm{NOTCH}_{1}$ and $M Y D 88$, while in patients harbouring $\mathrm{SF}_{3} \mathrm{~B}_{1}$ mutation the tendency to lower median TFTT was revealed $(p=0.08)$. Interestingly, the significant difference in median TTFT was observed in groups of men and women, showing the better outcome in female patients (median 10 vs 28 months, $p=0.01$ ). conclusion: The presence of biological and genetic factors of unfavorable prognosis is reflected in more aggressive course of the disease.

Keywords: chronic lymphocytic leukemia, NOTCH1, SF3B1, MrD88, time to first treatment

52. Transplantation waiting list in Patients with Chronic Renal Failure in compare with Polycystic Kidney Disease

Jerko Arambašić ${ }^{1}$, Marko Lovrić1, Barbara Dumančić ${ }^{1}$ Katarina Badak', Anja Comišček', Lada Zibar ${ }^{1,2}$

${ }^{1}$ Faculty of Medicine, J. J. Strossmayer, ${ }^{2}$ Clinical hospital Osijek

Background: Considering number of population, Croatia is leading country in the world in organ tranplantation (liver and kidney). That's main reason why we have decided to see statistical difference between patients with polycystic kidney disease and patients with chronic renal failure. Methods: Statistical analysis of data in patients with polycystic kidney disease and patients with chronic renal failure, who were on the list for kidney transplantation. Results: Patients who had polycystic kindey disease and were on the list for kidney transplantation got kidney in shorter period of time in compare with patients with chronic renal failure. Conclusion: Patients who have polycistic kidney disease were considered for kidney tranplantation even before the 
patients with chronic kidney failure, and that is the main reason why they were shorter period of time on waiting list.

Keywords: Polycystic Kidney Disease, Chronic Renal Failure, kidney, transplantation, kidney transplantation

53. Treatment Modalities and Results of Patients with Clioblastoma Multiforme

EBUBEKIR UCAR ', Rasim Meral,MD 2

' Faculty of Medicine, Istanbul University, Istanbul, 34093, Turkey, 2 Professor in Radiation Oncology, Oncology Institution, Istanbul University, Istanbul, 34093, Turkey

Background: Glioblastoma multiforme (GBM) is a disease which has poor prognosis. Recently, prognosis of GBM patients' improved with developments of micro-surgery and addition of Temozolomide (TMZ) chemotherapy to adjuvant radiation therapy in a concomitant and adjuvant setting. Aim of this study was to evaluate the role of the adjuvant radiation therapy (RT) with concurrent chemotherapy (ChT) after maximal safe resection in patients with GBM. Methods: The researchers have the charts of all patients with CBM diagnosed at Oncology Institution, Istanbul University in 2010. We retrospectively reviewed after follow up 20 months (range 1-60 months). Collected data analyzed for RT, ChT, age, sex and tumor control. Results: Ten patients (4 female, 6 male; median age 53; range 32-63 years) were recruited for this study. Surgery (partial $30 \%$, total $50 \%$, and biopsy $20 \%$ ) followed by postoperative RT (mean dose $\mathrm{xx}$ Gy) was the treatment of choice with concurrent TMZ. Objective tumor control was achieved in 5/10 patients. Additional ChT was given to 2 patients for progressive disease with secondary surgery in only one of them. Conclusion: Despite unfavorable prognosis of patients with GBM in the past, improvement of treatment modalities and particularly additional TMZ ChT increased treatment results considerably as shown in this study with high objective responds rates.

Keywords: glioblastoma multiforme, prognosis, resection, temozolomide

54. Estimation of renal function in male and female patients with diabetes mellitus type 2

Horozić Džan', Babić Nermina', Avdagić Nesina', Vanis Nedim', Sokolović Emir'

${ }^{7}$ Faculty of Medicine, Sarajevo, 71000, Bosnia and Herzegovina

Background: Diabetes is major risk factor for renal disease. Some studies have shown that healthy women have lower risk on developing renal disease compared to men. This risk evens in diabetic patients. The glomerular filtration rate (CFR) is the best overall measure of the renal function. The aim of this study was to investigate gender difference in GFR defined with both creatinine clearance $(\mathrm{CrCl})$ and MDRD formula in patients with type 2 diabetes mellitus (T2DM). Methods: This study included 72 patients with T2DM. They were divided into two groups, male $(n=32)$ and female $(n=40)$. Routine biochemical parameters, blood glucose, $H b A 1 c$, serum creatinine, urea, urine creatinine, urine albumin levels, 24 hour urine collection were measured in all subjects. In every patient, GFR was estimated with both MDRD formula and $\mathrm{CrCl}$. Results: There was no age difference between the groups. Study showed no significant difference in blood glucose $(p=0,138), \operatorname{HbAlC}(p=0,400)$, urea $(p=0,421)$ and albumin $(p=0,371)$ levels between the groups. There was no significant difference in GFR calculated with MDRD formula between male and female group $(91,04 \pm 41,19 \mathrm{ml} / \mathrm{min}$ vs. $77,94 \pm 30,14 \mathrm{ml} / \mathrm{min} ; p=0,138)$. GFR values estimated by measuring $\mathrm{CrCl}$ showed no significant difference between male and female group $[87,00(64.50,145.50)$ $\mathrm{ml} / \mathrm{min}$ vs. $84.00(66.00,144.00) \mathrm{ml} / \mathrm{min} ; \mathrm{p}=0.902]$. Also, there were no significant difference between GFR values estimated by MDRD and by $\mathrm{CrCl}$ in male $(p=0.733)$ and female $(p=0.073)$ group. Conclusion: Our study showed that there was no significant difference in GFR between male and female patients with T2DM. Also, there was no difference between GFR values estimated by MDRD and GFR values calculated with $\mathrm{CrCl}$ in both male and female group. Therefore, according to this study both groups of patients have equal risk of developing renal disease.

Keywords: diabetes, renal function, MDRD, creatinine clearance

55. Analysis of nutritional status of preschool children and impact of collective diet on nutritional status

Mina Poskurica ${ }^{{ }^{*}}$, Djordje Stevanovic ${ }^{1}$, Nela Djonovic ${ }^{1}$ Mileta Poskurica ${ }^{1,2}$, Dalibor Stajic

' Faculty of Medical Sciences, University of Kragujevac, Serbia, Clinic of Nephrology, Clinical Center Kragujevac, Serbia

Background: The main goal of proper nutrition is to provide children adequate intake of optimum amount of nutritients. collective diet represents a correcting factor of irregularity in family nutrition.Overweight in children represents a predictor of obesity in future age.The aim is to evaluate nutritional status of preschool children and impact of collective diet on nutritional status. Methods: Retrospective study was conducted in "Bambi" kindergarten,Kragujevac, on 185 children,divided into four groups by age(from age 4 to 7$) .98(53 \%)$ were boys, $87(47 \%)$ were girls, aproximatly equally divided in groups.From kindergarten,data was collected about weight and height in all four quartals in year 2015/2016.From Institute of Public Health data was collected about energetic values of every meal and macronutritient used,wich were the same for all the children.Body mass index(BMI)was used to assess childrens weight status.Data was analyzed by SPSS statistical software(tests used:Chi-square,Mann-Whitney test and descriptives). Results: Percentage of underweight children in all four groups were in average $5.95 \%$ (by quartals $10.6 \%, 6.4 \%, 5 \%$, $1.8 \%$ ), with normal BMI $77.775 \%(78.1 \%$, $80 \%, \quad 78.2 \%$, $74.8 \%)$,overweight $9.12 \%(7.5 \%, 7.2 \%, 9.5 \%, 12.3 \%)$ and obesse $7.125 \%(3.8 \%, 6.4 \%, 7.3 \%, 11 \%)$.Nutritional status had no statisticly significant difference in examined groups in nor one quartal.No statistical difference was found between groups of boys and girls. In all four groups, the increase of both weight and BMI was spotted,with very high statisticly significant difference of weight $(p=0.001, r=0.19)$ and $\operatorname{BMI}(p=0.000, r=0.295)$ between first and fourth quartal.Income in weight and BMI corresponded with energy values of meals.The highest income in weight was noticed between first and second quartal,with statistical significance $(p=0.009, r=0.15)$, when the value of energy intake was the highest. Conclusion: About $20 \%$ of examined children did not have adequate nutritional status,more toward overweight category.Considering the fact that the energy intake was the same for all the children,we find the importance of eating and healthrelated habits children adopt in family environment, and that the collective diet is the correcting factor of irregularity in family nutriotion.

Keywords: preschool children, collective diet, nutritional status

\section{Impact of eating habits on nutritional status and fat tissue} percentage in students

Djordje Stevanovic ${ }^{{ }^{*}}$, Mina Poskurica ${ }^{1}$, Nela Djonovic 1 , Mileta Poskurica ${ }^{1,2}$, Dalibor Stajic

' Faculty of Medical Sciences, University of Kragujevac, Serbia, 2 Clinic of Nephrology, Clinical Center Kragujevac, Serbia

Background: Bad eating and health-related habits, common in students, lead not only to disturbed nutritional status, high fat tissue percentage, and eventualy to obesity, but are important risk factors for significant number of chronic diseases. The objectives of the research were to determine the prevalence of overweight, obesity and high fat tissue percentage in students, as well as the relationship between nutritional status and eatinig and healthrelated habits. Methods: A cross-sectional study was conducted on 262 students (130 males, and 131 females) of Faculty of Medical Sciences,University of Kragujevac,Serbia.Participants were divided into six groups, by year of study(with aproximatly equal number of males and females in groups).Students filled out a self-reported questionnaire considering their eating and lifestyle habits.Students height,weight,body mass index (BMI),body fat percentage $(\mathrm{BF} \%)$, visceral fat (VF) and sceletal muscles percentage (SM\%) were measured.Data was analyzed by SPSS statistical software 
(tests used: Chi-square,Mann-Whitney test and correlation) Results: of total 262 participants: $5.3 \%$ were underweight, $75.6 \%$ had normal BMI, $14.9 \%$ were overweight and $4.3 \%$ had obesity; $6.5 \%$ had low $\mathrm{BF} \%, 58.4 \%$ were normal range, $22.9 \%$ with high $\mathrm{BF} \%$, and $12.2 \%$ with very high $\mathrm{BF} \%$. Comparing to the group with BMl 25 , the group with BMl 25 reported higher usage of white bread $(p=0.016)$, fast food $(p=0.004)$, sweets and snacks (both $p=0.001), \operatorname{Tv} / \operatorname{Pc}(p=0.000)$, more frequent skipping meals $(p=0.002)$ and less frequent phisysical activity $(p=0.001)$. The moderate strong positive correlation was found between $\mathrm{BMI}$ and $\mathrm{BF} \%$ $(r=0,363)$. Students of fifth and sixth year of studying comparing to those in first four years had significantly higher values of $B M I$ $(p=0,019), \quad B F \%(p=0.022), \quad V F(p=0.001)$, and significantly lower values of SM\% $(p=0.044)$. Conclusion: The fact that $25,7 \%$ of students are overweight/obesse and $35,1 \%$ has high/very high $\mathrm{BF} \%$, considering frequency of bad eating and health-related habits and its obvious impact on these numbers and growing prevalence as we go through the School of Medecine, emerges a need for changing life habits in these population to prevent various chronic disseaes.

Keywords: Obesity, students, eating habits

\section{DIAGNOSTIC DILEMMA IN DIAGNOSING ACUTE INTERMITTENT PORPHYRIA}

Malik Ejubović

' Department for Internal medicine, Cantonal hospital Zenica, Bosna and Herzegovina

Background: Porphyria is a group of diseases in which the precursor molecules of porphyrin or porphyrin itself accumulates in the body. A type of the disease which has an impact on the nervous system is called an acute porphyria. Acute intermittent porphyria (AIP) is a rare autosomal dominant metabolic disorder which affects the production of hem, an oxygen-binding part of hemoglobin. It is manifested by the attacks that last for several days characterized by abdominal pain, nausea, vomiting, bowel problems, urinary disorders, tachycardia, high blood pressure, general restlessness, muscle weakness or cramps. In order to diagnose this disease, apart from these symptoms, it is necessary to prove the presence of hem precursors in the urine (deltaamino-levulinic acid and porphobilinogen). In this paper, we present a case of a 36-year old female patient hospitalized for abdominal pain, nausea, vomiting, general weakness and arthralgia at the Department of Internal medicine, Cantonal hospital Zenica. Laboratory: Leu-14.35; Er: 3.76; Hgb: 11.6; Hct: 0.34; MCV: 90.9; MCH: 30.7; MCHC: 33.8; RDW: 11.1; Plt: 320; Urea: 6.8; Creatinine: 84; Sodium: 137; Potassium: 3.8; Chlorides: 106. Physical examination: eupnoic, tachycardic (100/min), epigastric pain, TA $130 / 90 \mathrm{mmHg}$. Ultrasound: enlarged liver and pancreas, kidneys with dilated renal tubules. Due to the specific clinical presentation, symptoms and the laboratory findings, an acute intermittent porphyria was suspected to be our patient's diagnosis. However, family history revealed no porphyria or other hereditary diseases. Patient was treated with proton pump inhibitors, analgesics, antiepileptics and glucose solutions. She was discharged from the hospital recovered and with the recommendation for further analysis. Given the diversity and nonspecificity of the symptoms that are present AIP, it is easy to misplace this diagnosis. It is important to obtain an adequate history and to think about porphyria in general, in order to confirm this disease.

Keywords: porphyrin accumulation, acute intermittent porphyria, delta-amino-levulinic acid, porphobilinogen

\section{Poster session I:}

58. Antileukemic Effect of Cyclohexyl-Functionalized Diamine $\mathrm{Pt}$ (II) And Corresponding Pt(IV) Complexes

MIRJANA ARSENIJEVIC' ${ }^{{ }_{1}}$, Sonja Misirlić Denčić, Asst Prof ${ }^{2}$; Anđelka Isaković, TA²

School of Medicine, University of Belgrade, Belgrade, 11000, Serbia, ${ }^{2}$ Institute of Medical and Clinical Biochemistry, School of Medicine, University of Belgrade, Belgrade, 11000, Serbia
Background: Blood cancers are expected to account for $9.8 \%$ of the cancer deaths (USA; 2016). As chemotherapy is used to treat most types of leukemia, the search for new and more effective drugs is ongoing. We investigated cytotoxic effects of cyclohexylfunctionalized diamine Pt(II) and corresponding Pt(IV) complexes on leukemic HL-60 cell line. Methods: The number of viable $\mathrm{HL}-60$ cells was determined using acid phosphatase and LDH assays. Flow cytometry analysis of HL-60 cells stained with appropriate fluorochromes was used in order to determine $\mathrm{O}_{2}{ }^{-}$and overall ROS production, caspsases 8,9 and 3 activation and phosphatidyl serine externalization. Results: Both investigated Pt complexes significantly reduced the number of viable $\mathrm{HL}-60$ cells, with $\mathrm{Pt}(\mathrm{II})$ complex being more efficient. The LDH test indicated that $24 \mathrm{~h}$ treatment with $\mathrm{Pt}(\mathrm{II})(24 \mathrm{~h} ; 5 \mathrm{MM})$ resulted in less cell membrane damage than treatment with Pt(IV) complex $(24 \mathrm{~h} ; 18 \mu \mathrm{M})$. Pt(II) caused marked caspase- 8 and moderate caspase-9 activation (2h), later (4h) followed by increased $\mathrm{O}_{2}^{-}$and overall ROS production. $\mathrm{Pt}(\mathrm{IV})$ caused weaker caspase-8 activation that later (4h) caused more intense overall ROS production compared to Pt(II). Caspase-3 activation was more pronounced following Pt(II) compared to the $\mathrm{Pt}(\mathrm{IV})$ treatment (12h). Additionally, Pt(II) caused the increase in $\mathrm{Ann}^{+}$cells from $6.2 \%$ in untreated control to $>50 \%$ following treatment (24h). Pt(IV) treatment (24h) caused both the increase in apoptotic Ann $n^{+}$cells and necrotic $\left(\mathrm{Ann}^{-} / \mathrm{Pl}^{+}\right)$cells $(230 \%$ in comparison to $1.0 \%$ in control) confirming the LDH result. Conclusion: $\mathrm{Pt}$ (II) induces apoptotic leukemic cell death in the concentration several times lower compared to corresponding $\mathrm{Pt}$ (IV) complex. Pt(IV) induces mixed apoptotic/necrotic HL-60 cell death. Having in mind that Pt(IV) complex is reduced to $\mathrm{Pt}(\mathrm{II})$ complex in bloodstream in order to achieve its biologically active form, $\mathrm{Pt}(\mathrm{II})$ complexation with this type of organic ligand might be a better option for achieving antileukemic effect.

Keywords: cytotoxicity; Pt complexes; oxidative stress; apoptosis; necrosis.

\section{Decision Tree Classifiers for Breast Cancer Detection}

LAYLA ABDEL-ILAH ${ }^{*}$, Ahmed Osmanović 1 , Sabina Halilović 1. Dževida Tarakčija 1, Fatima Mrkulić 1, Adnan Hodžić 1 ,Jasmin Kevrić

- International Burch University, Sarajevo, 71200, Bosnia and Herzegovina

Background: Breast cancer is the most frequently diagnosed cancer and the leading cause of cancer death among females, accounting for $23 \%$ of the total cancer cases and $14 \%$ of total cancer deaths. Weka is a collection of machine learning algorithms for data mining tasks, and contains tools for data pre-processing, classification, and visualization. Aim: Classification of breast cancer cases as malignant or benign by decision tree classifiers implemented in Weka software since majority of previous studies used artificial neural networks for breast cancer detection. Methods: Samples are obtained from the Wisconsin Breast Cancer Database (WBCD), which is open access dataset. The dataset contains 699 samples and each sample has 9 attributes representing 9 characteristics of breast fine-needle aspirates (FNAS). This experiment includes a comparison among the obtained accuracy when using three decision classification algorithms in Weka environment: C45, REPTree, and Random Forest. In addition, this experiment compares the obtained accuracy when using 70-30, and 60-40 distributions. Results: By implementing 70-30 distribution, C45 algorithm achieved $94.7 \%$ accuracy, while REPTree achieved $92.4 \%$, and Random Forest achieved $97.1 \%$. Implementing 60-30 distributions resulted in $93.9 \%, 93.2 \%$, and $96.1 \%$ accuracy achieved by C45, REPTree, and Random Forest respectively. Conclusion: Results obtained from this study show that decision tree algorithms are strong candidates for breast cancer detection. Random Forest proved to be the most suitable decision tree algorithm for breast cancer classification because it achieved the highest accuracy in both distributions.

Keywords: breast cancer, Weka, Decision Tree Classifiers, C45, REPTree, Random Forest. 
60. Temporal dynamics of the phase shift between orthogonal components of the amplitude of the laser radiation scattered by skin tissue to diagnose the prescription of death coming

Oleksandr Pavliukovych', Viktor Bachinskiy', Nataliia Pavliukovych

7 Higher State Educational Establishment of Ukraine "Bukovinian State Medical University", Chernivtsi, 58000

Background: The basic tasks of practical forensic medical experts are establishing the cause of death and the prescription of its coming that is very complicated due to the absence of circumstances and objective data which would be able to point out a possible time of its coming. This research is aimed at detecting temporal spectral changes of depolarizing laser irradiation by means of sections of the human skin tissue to diagnose the time of death coming. Methods: Sections of the skin tissue of people who died as a result of mechanical asphyxia (" $A$ ") - 100 defuncts and massive blood loss ("B") - 98 defuncts. Sections under study were placed in a laser unit where a collimated beam radiation was carried out of He-Ne laser. Results: Analysis of experimentally obtained findings of the structure of a depolarized component and temporal evolution of laser radiation scattered by the skin tissues has revealed: structural hetergeneity of two-dimensional distributions $\Delta(\mathrm{x}, \mathrm{y})$ that are formed by a combination of predominantly small-scale sites values of the degree of depolarization, arranged randomly in the shear plane of the skin tissue; an increase of the level of the depolarization of scattered radiation and a transformation of the sizes of sites of identical values $\Delta(x, y)$ in a direction of enlarging geometrical dimensions with a time increase after death coming. Revealed specific characteristics of a transformation of polarized layer radiation that passed through the blood saturated laser of the skin tissue may be associated with the following features of its morphologic structure. Conclusion: Use of a three-dimensional parameter of the depolarization of laser radiation scattered by sections of the biological tissues of the human body may be used for a search of new criteria for an expansion of possibilities of diagnosing the time of death coming.

Keywords: death, asphyxia, blood loss.

\section{TURNER SYNDROME IN CHILDREN OF NORTHERN BUKOVINA \\ Mariana Ryznychuk \\ 'Bukovinian State Medical University, Ukraine}

Background: Turner syndrome refers to orphan diseases. From 17 to 25 cases of this syndrome are firstly diagnosing every year in Ukraine. Besides monosomy, pathology of $X$ chromosome $(60-70 \%)$ in Turner syndrome may be caused by a wide genetic polymorphisms (variants of mosaicism, deletions of short and long arm of the $X$ chromosome, isochromosome, ring chromosomes (30-40\%)). Methods: The study involved 8 patients with Turner syndrome with monosomy of the $X$ chromosome and 1 child with mosaic variant of the syndrome, who were registered in the Regional Children's hospital of Chernivtsi. The average age at the moment of the survey was $13,78 \pm 0,11$ years old. A medicalgenetic counseling, somato-genetic testing, clinical and genealogical analysis were performed. Results: In all children occurred low growth $(100 \%)$. In $56 \%$ of children was found hypergonadotropic hypogonadism. Sporadic puberty was only in one child $(11 \%)$ with the mosaic variant of syndrome. The main complaints of girls were growth retardation and lack of secondary sexual characteristics. There were such basic phenotypic violation in research: antimongoloid eyes (89\%), ptosis (78\%), ocular hypertelorysm (89\%), epikant (33\%), strabismus $(22 \%)$, micrognathia $(22 \%)$, high gothic palate $(89 \%)$, cleft soft palate $(11 \%)$, abnormal structure of ears $(89 \%)$, low growth of hair on the neck $(100 \%)$, short neck $(100 \%)$ barrel chest $(89 \%)$, coarctation of the aorta $(11 \%)$, mitral valve prolapse $(44 \%)$, hypertelorysm of nipples $(89 \%)$, scoliosis $(33 \%)$, brachydactyly (100\%), short fourth and fifth metacarpal bones (11\%), nail dysplasia $(11 \%)$, sandal cleft $(33 \%)$, delayed skeletal maturation $(56 \%)$, multiple pigmented nevi $(22 \%)$, overweight $(67 \%)$. Average growth was $-131,9 \mathrm{~cm}$, growth retardation $-4,2 \pm 0,22$ SDS, bone age $12,10 \pm 0,12$ years, average weight - 34,9 kg. Conclusion: Phenotypic features of Turner syndrome are overlooking by pediatricians and children gynecologists, suggesting the need for careful attention to girls with delayed growth and sexual development.

Keywords: Turner syndrome, growth retardation, karyotype, physical development.

62. The Impact of II-12b Gene Polymorphism on Kidney Function after Transplantation

ISIDORA BANJAC ', Vladimir Perović

' School of Medicine, University of Belgrade, Serbia

Background: IL-12 may affect the outcome of renal transplantation and lead to acute rejection, due to its proinflammatory functions. $I L-12 B$ gene is coding p40 subunit of $I L-12$. There are a few polymorphisms in its sequence that could alter the gene expression or its protein structure. Results from the previous research on $1188 \mathrm{~A}>\mathrm{C}$ polymorphism showed great variability in $I L 12 B$ expression profile.The aim of this study was to determine the frequencies of alleles, genotypes and allele carriers of $I L-12 B$ gene $1188 \mathrm{~A}>\mathrm{C}$ polymorphism in patients after kidney transplantation and further evaluate the correlation with acute rejection (AR) and delayed graft function (DGF). Methods: A total of 152 patients were included in this retrospective cohort study. Real-time polymerase chain reaction was used to determine the genotype which was then evaluated in relation to acute rejection and delayed graft function. Statistical significance was analyzed by Pearson's Chi-square test and Fisher's exact test. Results: The most frequent genotype among 152 patients was AA (69.1\%), then CA $(28.3 \%)$ and CC (2.6\%). Distribution of alleles A and C was $83.2 \%$ and $36.8 \%$, respectively. There was no statistical significance in distribution of IL-12 genotypes and alleles with respect to AR or DGF. Delayed graft function occurred in 18 out of 41 allele C carriers while in group of non-allele $C$ carriers this complication was present in 29 out of 89 subjects. There was statistically significant difference in distribution of allele $C$ carriers between groups of patients with and without DGF $(p=0.035,0 R=0.45,95 \%$ confidence interval $(0,21-0,95))$. Conclusion: In this study, association between $I L-12 B$ gene $1188 A>C$ polymorphism and delayed graft function was demonstrated in renal transplant recipients. Allele $C$ carriers confer higher risk in developing delayed graft function after kidney transplantation while AA genotype represents protective factor. However, we observed no association between this polymorphism and acute renal rejection.

Keywords: IL-12B; Polymorphism; Acute Rejection; Delayed Graft Function

\section{Brain abscess in patient with Rendu-Weber-0sler disease}

Nives Šalek ${ }^{(1)}$,Dora Savanović(1), Ana Vidić (1), Mirna Rozić(1) $^{(1)}$ Anja Tomić(1) Silva Zupančić-Šalek ${ }^{(1,2)}$

1 University J.J.Strossmayer Osijek, School of Medicine, Osijek, 31000, Croatia, ${ }^{2}$ University Hospital Centre Zagreb $\circlearrowright$ University of Zagreb, School of Medicine Zagreb, Zagreb 10000, Croatia

Background: The Rendu-Weber-Osler disease, also known as hereditary haemorrhagic telangiectasia (HHT), is a rare autosomal dominant disorder that affects blood vessels formation throughout the body and results in a tendency for bleeding. Diagnosis is made according to Curacao Criteria: telangiectasia on the face, hands and oral cavity; recurrent epistaxis; arteriovenous malformations with visceral involvement; family history. If three out of four criteria satisfied, the patient is consider to have definite HHT. The incidence in the general population is of 12/100000 and has a homogenous race and gender distribution. The symptoms of OWRD are often unrecognized and many patients may go undiagnosed Case presentation: We report a case of 61 year old female with HHT who developed liver hematoma and secondary infection of hematoma. The bacteria was E.colli. Liver resection was done on February 2015. In July 2016 she developed right- sided motor witness with headache and aphasia. CT scan demonstrated a frontotemporal mass and a brain abscess was strongly suspected. The brain abscess was surgically removed and E.colli was proved in cultures. She recovered completely. 
Conclusion: Brain abscess usually develops from a contiguous focus of infection, most often from infections in the middle ear, mastoid cells or paranasal sinuses and less often from dental infection and trauma or haematogenous dissemination. Our patient's brain abscess could have been secondary to a liver abscess. The other possibility is the development of PAVMs of the lung which remain for years unrecognised. It is known that asymptomatic PAVMs are not inactive. Patients suffering from HHT belong to the group of high risk of development of PAVM, which if not treated on time may cause a brain abscess.

Keywords: Rendu-Weber-Osler disease, brain abscess, telangiectasia

Application of Methods of Laser Polarimetry In Early Detection of Hemorheological Disorders In Patients With Coronary Artery Disease, Diabetes Mellitus And Anemia Pavliukovych N.D., Pavliukovych O.V.

1 Higher State Educational Establishment of Ukraine "Bukovinian State Medical University", Chernivtsi, 58000, Ukraine

Background: Laser radiation during interaction with biological tissue can be absorbed and dissipated. These processes carry information on micro- and macrostructure of biological substances. Methods: 120 patients with coronary artery disease (CAD), diabetes mellitus type 2 (DM) and anemia were under investigation. They were randomized into groups according to comorbidity (CAD and DM; CAD, DM and aneia) and degree of anemia severity. For objective assessment of functional state of erythrocytes membrane laser polarimetry of erythrocyte suspension (ES) smear was applied. To assess coordinate distributions of the random variables erythrocytes histograms were used; then set of statistical points of the $1^{\text {st }}-4^{\text {th }}$ grades (medium, variance, asymmetry, curtosis) was calculated. Results: Coordinate and probability intensity distributions of Fourier spectrum of the ES laser image of control group had symmetrical "bell-like" appearance. Intensity distribution of Fourier spectrum of the ES laser image of patients with CAD and DM was uneven, and the histogram was transformed into asymmetric dependence due to growth of anisotropic component of erythrocytes membrane, conditioned by conformational changes of membranes protein structure. More clearly such transformations are observed during investigation of the Fourier spectrum distribution of the ES laser images of patients of other studied groups and the degree of the erythrocytes anisotropy remained high. This served as the basis of heterogeneity of erythrocyte membranes structure due to investigated comorbidity. Correlation analysis showed direct relationship between level of fasting glucose and anisotropy degree of ES of patients with CAD and DM, while the level of hemoglobin was negatively associated with values of asymmetry and kurtosis. Conclusion: Methods of laser polarimetry of erythrocytes smear might be used for early diagnosis of structural changes of erythrocytes membrane in patients with comorbid course of CAD, DM and anemia when they can not be objectified by means of traditional methods

Keywords: coronary artery disease, diabetes mellitus type 2, anemia, laser polarimetry, erythrocyte

65. Arrhythmogenic Right Ventricular Cardiomyopathy and the Importance of Genetic Testing- A Case Report

IVAN ŠEVELJEVIĆ ${ }^{1}$, Luka Vranić1, Ivan Vuksan', Andrej Belančić ${ }^{1}$, Zlatko Cubranić2

' University of Rijeka, Faculty of Medicine, Rijeka, 51000, Croatia, ${ }^{2}$ Department of Cardiovascular Diseases, Clinical Hospital Centre Rijeka, University of Rijeka, Faculty of Medicine, Rijeka, 51000, Croatia

Background: Arrhythmogenic right ventricular cardiomyopathy (ARVC) is an inheritable heart muscle disease. This rare structural disease predominately affects the right ventricle (RV), causing the fibrofatty replacement of the myocardium. ARVC is an important cause of ventricular arrythmias and sudden cardiac death (SCD), especially among young patients Case presentation: 42 year-old male patient presented with fatigue, lightheadedness, nausea and heart palpitations that began the day before and continued onward. Past medical history revealed hyperlipidemia and positive family history of heart diseases. ECG showed ventricular tachycardia with broad QRS complexes that persisted despite the antiarrhythmic therapy. Subsequently, the patient became hemodinamycally unstable and had to be cardioverted to synus rythm. Urgent coronarography showed normal coronary arteries. Inverted $\mathrm{T}$ waves in precordial leads and prolonged terminal activation delay (.55 milliseconds) were present in repeated ECG's. 24-hour Holter monitoring was conducted showing nonsustained ventricular tachycardia. Echocardiography showed slightly enlarged right ventricle (4-chamber diameter of $55 \mathrm{~mm}$ and RV:LV ratio of 1.0 without visible contraction failures). Heart MRI revealed regional RV hypokinesia with RV ejection fraction of $40.4 \%$ which meets the criteria for ARVC. Due to risk of the possible SCD implantable cardioverter defibrilator (ICD) was implanted and medical therapy with $\beta$-blockers, ACE inhibitors and statins was proposed. Blood sample taken for genetic testing showed DSP gene code mutation E1265X, confirming the diagnosis of ARVC. Conclusion: The present report describes an interesting case of ARVC and demonstrates the importance of genetic testing in diagnostic protocol. Genetic testing proves its quality when it comes to establishing diagnosis of the disease among presymptomatic family members and proposing genetic counseling. Moreover, this case presents the importance of ICD implantation in ARVC managment which is recommended for implantation in patients with documented sustained VT or VF.

Keywords: Arrhythmogenic right ventricular cardiomyopathy; Genetic testing; Implantable cardioverter defibrilator

66. Brugada Syndrome Revealed After Routine Ergometry Test - A Case Report

LUKA VRANIĆ' 1 Ivan Vuksan', Ivan Ševeljević1, Zlatko Čubranić ${ }^{2}$

'University of Rijeka, Faculty of Medicine, Rijeka, 51000, Croatia, Department of Cardiovascular Diseases, Clinical Hospital Centre Rijeka, University of Rijeka, Faculty of Medicine, Rijeka, 51000, Croatia

Background: Brugada syndrome is autosomal-dominant inherited sodium channelopathy that can lead to life-threatening arrhythmias. It is rare disease on structurally normal heart with clinical presentation that spans from being asympthomatic syncope, palpitations, nocturnal agonal respirations, sustained ventricular tachycardia, ventricular fibrillation even to sudden cardiac death (SCD). Case presentation: 34-year old male patient presented to nephrologist with symptomatic attacks of high blood pressure. During medical examination, ergometry test showed non-sustained ventricular tachycardia, ventricular extrasystoles, "R-on-T" phenomenon and discreet Brugada ECG pattern type 1 : right bundle branch block and ST-elevation in $V_{1}$ and $V_{2}$. Ventricular excitability decreases as the exertion drops. At admission, blood pressure was 145/100, heart rate 105/min while laboratory findings were normal. Urgent coronarography showed normal coronary arteries. Echocardiography and heart MRI haven't showed any structural changes or abnormalities. Due to suspicion of Brugada syndrome, ECG was repeated which hasn't revealed Brugada pattern, but repositioning $V_{1}$ and $V_{2}$ leads to second intecostal space has discreetly unmasked Brugada pattern type 1. Provocation test with ajmaline was performed but progression of initial ECG Brugada pattern wasn't observed. Severe ventricular excitability was revealed during electrophysiological study. Programmed electric stimulation and ajmaline application triggered hemodynamically unstable sustained ventricular tachycardia which had to be cardioverted to sinus rythm. Due to assessment of the possible SCD, implantable cardioverter defibrilator (ICD) was implanted. Conclusion: We present an interesting case of Brugada syndrome with ventricular excitability triggered in exertion. Typical presentation of this syndrome are fatal arrhythmias in rest, commonly while sleeping. Moreover, this case presents therapeutic dilemma whether implant ICD or not. According to 2015 ESC guidelines ICD should be implanted only in patients with ECG Brugada pattern type 1 who survived SCD or have a history of 
unexplained syncope and/or documented spontaneous sustained ventricular tachycardia'.

Keywords: Brugada syndrome; Electrophysiological study; Ergometry test; Implantable cardioverter defibrilator

\section{Factor VIII inhibitors in mild haemophilia A}

Anja Tomic ', Mirna Rozic ', Nives Salek ', Dora Savanovic ', Ana Vidic ', Silva Zupancic-Salek ${ }^{2}$

' University f.j.Strossmayer Osijek, School of Medicine, Osijek, 31000, Croatia, ${ }^{2}$ University Hospital Centre Zagreb 8 University of Zagreb, School of Medicine Zagreb, Zagreb 10000, Croatia

Background: Haemophilia A is the most common congenital severe bleeding disorder and is a result of a deficiency in a clotting protein called factor VIII. Factor VIII deficiency is an X-linked recessive disorder occurring in 1 in every 5000 male births. FVIII replacement is effective unless a patient develops an alloantibody (inhibitor) against the exogenous FVIII, which can be detected either with routine laboratory testing or by clinical presentation (a patient experiences bleeding that does not adequately respond to hemostatic therapy). (1) On the other hand, inhibitors are an uncommon complication of mild haemophilia, occurring in 3-13\% of patients and usually arising during adulthood. Case presentation: We report a case of a male patient (D. D., 1977.), who was earlier diagnosed with mild form of haemophilia A. He was treated in 2007 in University Hospital Centres Traumatology department due to fracture of right radius in a car accident. Upon arrival, a treatment with concentrated substitutional FVIII was introduced, which resulted in development of high levels of inhibitors. Therefore, the patient was switched to a rFVlla therapy. Postoperative hemostasis was satisfactory. Three weeks after the operation, a paralysis $\mathrm{n}$. radialis and extensive hematoma appeared throughout the entire right arm, which is the reason why the patient was again introduced with intensive substitution therapy. Conclusion: Bleeding caused by cross reaction of inhibitors and FVIII can be a life threatening condition. Recombinant FVIla (which was used on our patient) and DDAVP (desmopressin, which stimulates the release of FVIII from storage sites) are the treatment of choice. (2) However, the optimal treatment regimen for the eradication of inhibitors and for the treatment of bleeding episodes have yet to be established.

Keywords: mild haemophilia, recombinant coagulation factor

68. Severe acute anemia - diffuse alveolar hemorrhage TINA SIRC, MD ${ }^{1,3^{*}}$, Jernej Bergant, $\mathrm{MD}^{2,3}$

' General hospital Jesenice, Slovenia, 2 University clinical center Ljubljana, Slovenia, 3 in cooperation with University Clinic for Respiratory and Alergic Diseases Golnik, Slovenia

Background: Anti-GBM antibody disease is rare disease with incidence of $0.5-1$ per million and represents a spectrum of diseases caused by circulating anti-GBM antibodies which affect the basal membranes of the alveoli and glomeruli. Typical symptoms are mild fever, fatigue and pallor, frequently as a result of sideropenic anemia. Respiratory symptoms are dyspnea and cough, which can rapidly progress to hemoptysis. With the use of modern diagnostic techniques the disease is rarely immuneserologically unproven, more often in younger men, smokers, with isolated pulmonary hemorrhage. Case presenatation: 22-year-old smoker presented with fatigue, which progressed over the week before admission to hospital. Until then, he was healthy. 4 months before his $\mathrm{Hb}$ was $158 \mathrm{~g} / \mathrm{L}$. Examination showed only pale skin and conjunctiva and some fine late inspiratory crackles. Lab tests showed severe microcytic, hypochromic anemia ( $\mathrm{Hb} 49 \mathrm{~g} / \mathrm{L}$ ), hemolysis was excluded. Urine tests showed blood in urine only once during 5 -week hospitalization. Sonography, gastroscopy and colonoscopy were normal. Chest X-ray and CT scan showed pronounced bronchovascular densities and interstitial thickening in the form of ground glass. Immunoserology was negative. Immunofluorescence analysis of lung biopsy tissue showed linear $\mathrm{IgG}(++)$ and $\mathrm{C}_{1}(+)$ deposits along the basal membrane of the alveoli $\rightarrow$ anti-GBM antibody disease. Conclusion: Seronegative anti-GBM antibody disease occurs in $2-3 \%$ of cases. (4) Inability to prove anti-CBM antibodies is most likely caused by technical limitations of used diagnostic methods. This is suggested by data from the literature, where many seronegative patients with diffuse alveolar hemorrhage became anti-GBM positive later in the course of the disease. Several similar cases remind us to suspect the anti-GBM antibody disease in case of unexplained diffuse alveolar hemorrhage despite the absence of anti-GBM antibodies in routine investigations.(5) Therefore in patients with suspected alveolar hemorrhage bronchoscopy, bronchoalveolar lavage and transbronchial biopsy for the immunohistology must be performed.

Keywords: anti-GBM, seronegative, alveolar, hemorrhage

\section{Clostridium difficile - associated diarrhea in a Serbian} University Hospital

Mirela Vatić ', Sena Aljušević ', Dolika Vasović ', TA Jasna Trbojević- Stanković ${ }^{2}$

1 Faculty of Medicine, University of Belgrade, 11000 Belgrade, Serbia, ${ }^{2}$ Clinic of Internal medicine, University hospital center " $\mathrm{Or}$ Dragiša Mišović- Dedinje",

Background: Clostridium difficile is the most frequent cause of nosocomial diarrhea. Infection caused by this bacterium causes a wide range of clinical manifestations, ranging from diarrhea to toxic megacolon, universaly reffered to as $C$. difficile-associated disease. Surveillance of $C$. difficile infection is compulsory in Serbian hospitals.The aim of this study was to define the incidence of Clostridium difficile-associated noscomial diarrhea and assess its clinical characteristics in patients treated at the Clinic of Internal Medicine in a University Hospital Center in Serbia. Methods: In this retrospective observational study data were collected for 79 patients ( 43 female, 36 male; mean age 72 years), who were admitted to the hospital between January 1, 2013 and December 31, 2015, and developed diarrhea with confirmed Clostridial A and/or B toxin in the stool. Data were analyzed with $t$, $\chi^{2}$, and Fisher's test, using the SPSS 20. Results: The overall incidence of $C$. difficile- associated nosocomial diarrhea was $0,007 \%$. Patients over 65 years had insignificantly higher mortality rate $(27 \%)$ than the younger ones $(16 \%)$. Comorbidities identified as risk factors for susceptibility to Clostridial infection included chronic renal failure $(74.68 \%)$, diabetes $(44.30 \%)$, heart failure $(41.77 \%)$, and malignancy $(27.85 \%)$. Previous use of antibiotics with broad-spectrum coverage (cephalosporins (30.00\%), carbapenems (14.00\%), and quinolones $(10.00 \%)$ ) was found to be an important predisposing factor for nosocomial diarrhea in the investigated group. Patients with lethal outcome had significantly lower levels of serum albumin and bicarbonate compared with those who recovered $(p<0.01)$. Disease presentation with high fever $\left(.38 .5^{\circ} \mathrm{C}\right.$ ) was significantly associated with mortality $(p<0.05)$. Conclusion: Patients who developed C. difficile- associated diarrhea tended to be older and suffering from certain comorbid conditions. Acidosis, hypoalbuminemia and high fever were associated with lethal outcome. Usage of antibiotics was associated with occurrence of Clostridial infection, therefore critical approach to antibiotic therapy is of paramount importance.

Keywords: Clostridium difficile, nosocomial diarrhea, usage of antibiotics

70. Efficiency of comprehensive therapy in patients suffering from chronic obstructive pulmonary disease with concomitant chronic acalculous cholecystitis

VIVCHARYK BOGDAN', Dudka Tetyana, Dudka Inna

1 Higher State Educational Establishment of Ukraine «Bukovinian State Medical University»

Background: To study the efficacy of Roflumilast, Ursodeoxycholic acid and Nucleinat in patients suffering from chronic obstructive pulmonary disease (COPD) with concomitant chronic acalculous cholecystitis (CAC). Methods: The study involved 40 patients with stage II COPD (GOLD 2, B) with concomitant CAC and 20 practically healthy individuals ( $\mathrm{PHI}$ ). Patients in the control group (group 2) received Berodual, Ursodeoxycholic acid for 30 days, in case of an 
infectious exacerbation of COPD - cephalosporin antibiotic therapy within 7 days. Group 1 (the study one, 20 people) received additional Roflumilast $500 \mathrm{mg}$ once a day, Nucleinat $500 \mathrm{mg} 3$ times daily for 30 days. Results: Figures of external respiration functions in dynamics of treatment in patients with COPD with concomitant CAC show higher efficiency of the proposed therapy too. In particular, the rate of forced expiration for the first second after treatment in patients of group 1 increased by $31,5 \%(p<0,05)$, while in patients in group 2 - by $14,0 \%(p<0,05)$ with the presence of reliable intergroup difference $(p<0,05)$. The content of malonic aldehyde in plasma after the treatment in group 1 decreased by 1,7 times $(p<0,05)$, while in group 2 - by 1,2 times $(p<0,05)$ with a reliable difference between groups $(p<0,05)$. Biochemical analysis of blood and bile for bilirubin after the treatment indicates its significant reduction in patients of group 1 - by 1,7 times in blood $(p<0,05)$ and $27,7 \%(p<0,05)$ in bile. In patients of group 2, due to the influence of the ursodeoxycholic acid, bilirubin in bile decreased by $7,8 \%(p<0,05)$, and the content of bilirubin in blood decreased by $13,0 \%(p<0,05)$. Conclusion: The use of roflumilast in combination with berodual, Ursodeoxycholic acid and Nucleinat in patients with COPD and in acute CAC promoted faster, than under conventional therapy (6-7 days), elimination of symptoms of both acute comorbid conditions.

71. Particularities of coagulation system in the chronic cholecystitis linked with somatoform autonomic dysfunction.

OLEKSANDRA SOLOVIANCHYK', Alena Antoniv

Bukovinian State Medical University, Ukraine

Background: Somatoform autonomic dysfunction (SAD) stays one of the major health problems in most countries because of its influence on the morbidity. Given the above, it is very important to study and improve all knowledges about the SAD, its pathogenesis on the background of accompanying diseases. The purpose of this research is to study particularities of coagulation and anticoagulation system in the chronic cholecystitis (CC) linked with SAD Methods: For this purpose 138 patients with accompanying SAD and CC were examined. Patients were divided into three groups: first - patients with $C C$ and $S A D$ for hypertensive type (HiperTT) (35 people); second - CC patients with hypotensive type SAD (HipoTT) (35 persons) and the third - CC patients with cardiac neurosis (CN) (38 people). The control group consisted of 30 practically healthy person (PHP). Total coagulation potential of the blood measured in terms of prothrombin time (PTT) fibrinolytic activity of plasma and content of fibrinogen in the blood plasma studied using sets of reagents firm "Danush Ltd" (Lviv). Results: Analysis of the study results of coagulation haemostasis showed that PTT of patients in second group was reduced by $21.9 \%$ compared with the rate in the PHP $(p<0.05)$. However, patients of 1st and 3rd comparison groups PTT had tended to increase, indicating the existence of the risk of hypercoagulability ( $p>0.05$ ). Researching of 3rd phase of coagulation hemostasis for content of fibrinogen in the blood suggested that index was significantly reduced in patients of all groups of observation: for patients of the 1 st group $-34.4 \%$, for group 2 - to $48.8 \%, 3$ group - to $26.6 \%\left(p_{1}-3<0.05\right)$ and significantly different when compared in terms of inter-group ( $p<0.05)$. Conclusion: Results of the study of haemostatic coagulation factors at patients with $C C$ with accompanying SAD for HiperTT and CN indicate at tendency to forming hypercoagulability due to failure of anticoagulation and fibrinolytic systems factors.

Keywords: SAD; Cholecystitis; Coagulation; Prothrombin

Sleep quality and sleep disturbances in hemodialysis patients - relationship with demographic characteristics and comorbidities

Sena Aljušević ', Mirela Vatić ', TA Jasna Trbojević Stanković ${ }^{2}$

- Faculty of Medicine, University of Belgrade, 11000 Belgrade, Serbia ${ }^{2}$ Clinic of Internal medicine, University hospital center " $\mathrm{Dr}$ Dragiša Mišović- Dedinje"
Background: Patients on dialysis often have poor quality of sleep, which is more prevalent than in the general population. However since the symptoms can be associated with kidney disease itself and/or response to dialysis treatment, sleep disorders are often unrecognized and therefore insufficiently treated. Aim: The aim of this study was to assess quality of sleep and prevalence of sleep disturbances in patients treated with maintenance hemodialysis, as well as to assess their relationship with demographic characteristics and comorbidities. Methods: A cross-sectional observational study involving 82 patients (49 men, age range $30-$ 85 years) treated with HD was undertaken in the University Hospital Center "Dr Dragiša Mišović" in Belgrade. Sleep quality was assessed with the Pittsburgh Sleep Quality Index (PSQI). Other relevant data were obtained from medical histories. Data were statistically analyzed with $X^{2}$ and $T$ test, using the SPSS 19.0. Results: The mean PSQI was $6.84 \pm 4.02$, and $50(61 \%)$ patients had poor quality of sleep (PSQ|>5). Average subjective sleep assessment rate was $0.98 \pm 0.87$, sleep latency $25.30 \pm 17.78$ minutes, sleep length $7.02 \pm 1.44 \mathrm{~h}$, and sleep efficiency $83 \pm 16 \%$. No statistically significant differences were found regarding these variables in relation to age, sex, employment status, smoking habit and hypertension. Patients with diabetes had significantly lower PSQI $(p=0.029)$, subjective sleep assessment rate $(p=0.036)$ and sleep efficiency $(p=0.022)$. The most prevalent sleep disturbances were spontaneous sleep interruption (70.37\%), >30 minutes needed for getting to sleep (68.58\%) and need to go to toilet $(55 \%)$. Sex and age were not significantly associated with frequency of certain sleep disturbances. Smokers significantly more often complained of spontaneous sleep interruption $(p=0.045)$ and cold $(p=0.008)$. Patients with diabetes significantly more often had bad dreams $(p=0.004)$. Conclusion: Patients on HD frequently had poor sleep quality. Smoking habit and diabetes were significantly associated with certain causes of sleep disturbances.

Keywords: sleep quality, sleep disturbances, hemodialysis

73. Serum lipid spectrum levels during chronic non-calculous cholecystitis depending on concomitant somatoform vegetative dysfunction type

HANNA HRYTSAK* , Alena Antoniv

'Bukovinian State Medical University, Ukraine

Background: Exacerbation of the chronic gastro-intestinal tract disease triggers progressive somatoform vegetative dysfunction (SVD) mechanisms, as listed: lipid peroxidation, oxidative protein modification, endogenous intoxication, metabolic acidosis. Thereby, clarification of the serum lipid spectrum (SLS) variability and its pathogenic role in course of the SVD development and progression during chronic non-calculous cholecystitis (CNC) requires further study. Methods: The study involved 138 patients with CNC and SVD. Patients were divided into three groups: first group - patients $(n=31)$ with CNC and hypertensive SVD type (HyperTT); second group - patients $(n=35)$ with CNC and hypotensive SVD type (HypoTT); third group - patients $(n=22)$ with CNC and cardiac neurosis (CN).Control group - 30 healthy individuals (HI) of similar age. Results: Serum total lipids (TL) value differed significantly depending on the concomitant SVD type: average rate in the first group had a downward trend although the significance was low ( $p>0.05$ ); second group's TL levels exceeded the normal value by $49.9 \%$ ( $p<0.05)$; third group's figures were slightly above the reference interval, the significance was low ( $p>0.05)$. Serum cholesterol levels indicated similar tendencies: significant increase by $44.3 \%(p<0.05)$ in the second group compared to the $\mathrm{HI}$; slight significant increase in the third group (by $11.8 \%, \mathrm{p}<0.05$ ); non-significant reduction in patients of the first group ( $p>0.05)$. Determination of the blood atherogenic lipoprotein levels showed a number of significant changes: lowdensity lipoprotein (LDL) rates were significantly lower in the first group (by $16.9 \%, p<0.05$ ); groups 2 and 3 showed significant LDL increase compared to the $\mathrm{HI}$ (by $52.7 \%$ and $18.1 \%$ respectfully). Blood levels of the very-low-density lipoprotein (VLDL) exceeded the control only in group 2 (by $62.7 \%, p<0.05$ ), whereas significant reduction of the VLDL rates was found in the 1st and 3rd groups (by $10.4 \%$ and $11.9 \%$ respectively, $\mathrm{p}<0.05$ ). Conclusion: The most 
unfavorable lipid profile was found in the patients with CNC and concomitant SVD of HypoTT, based on the serum levels of atherogenic lipid fractions. The mentioned above coincides with a significant intensification of lipid peroxidation processes in these

Keywords: Holecystitis; Atherogenic; Lipid.

74. State Insulin Resistance in Patients with Chronic Pancreatitis Against Obesity Treatment in the Dynamics of $\mathrm{L}$-Carnitine and L-Glutathione

SMANDYCH VITALII

Higher State Educational Establishment of Ukraine «Bukovinian State Medical University»

Background: Finding out the possible effect of L-carnitine and Lglutathione on clinical development of chronic pancreatitis (CP) in patients with obesity, oxidative and nitrositive stress, level of post prandial glycemia and the degree of insulin resistance. Methods: 80 patients with $\mathrm{CP}$ of a mixed etiology, pain form, relapsing course in the stage of moderate exacerbation with comorbid I degree obesity were examined. I degree obesity was diagnosed in case of body mass index (BMI) from 30 to $34,9 \mathrm{~kg} / \mathrm{m}^{2}$. To evaluate the efficacy of treatment by a random sign three groups of patients were formed. An average age was $(41,7 \pm 4,2)$. The group of general control included 30 practically healthy people (PHP) of acor responding age and sex. The control group (group 1 (C) - 25 individuals), the main group 2 (the group $2(0)-30$ idividuals). Results: The analysis of the therapy suggested in patients with $C P$ (group 0 ) on the development of the disease as compared to $C$ group has found the following results. Improved general state, reduced signs of asthenic-vegetative, intoxication syndromes, dyspepsia in patients of 02 and 3 groups were not ice don the 3-4 days since the initiation of treatment, while in the patients from $C$ group-only since the $10^{\text {th }}$ day. 2 weeks after initiation of the therapy asthenic-vegetative syndrome was absent in all the patients from 03 group (100\%), considerably less intensive was found only in 3 individuals $(10 \%)$ of 02 group, while in C group it remained in 11 patients $(36,7,0 \%)$. In the same term the majority of patients of 02 and 3 groups experienced less pain and heavy sensations in the epigastric area (in 25 (83,3\%) and $18(90 \%)$ respectively), and dyspeptic signs did not worry practically (28 patients (93,3\%) and 19 ones (95\%) respectively) observed in the majority of patients from C group and on the $15^{\text {th }}, 30^{\text {th }}, 60^{\text {th }}$ days of treatment. A positive effect of a comprehensive therapy with addition of Hepaval and L-carnitine on insulin resistance syndrome is are liable decrease of the body mass index in patients from 2 and 3 groups during the period of 60 days: on $7,5 \%$ and $8,1 \%$ respectively $(p<0,05)$. Conclusion: The suggested method of treatment of patients with chronic pancreatitis is against obesity including basic therapy supplied with L-glutathione and L-carnitine enables to eliminate clinical and biochemical in dices of chronic pancreatitis exacerbation. In addition by means of reduced antioxidant potential it enabled to decrease the degree of endogenic intoxication, oxidative and nitrose stress, normalize the level of glycemia and insulinemia, promote body mass loss.

Keywords: Chronic Pancreatitis, Obesity, L-Carnitine, L-Glutathione Metabolic Syndrome, Endogenous Intoxication, Insulin Resistance.

The Diagnoses and Monitoring of the Thyrotoxic Cardiomyopathy

VIRA SHUPER ${ }^{*}$, Sergij Shuper ${ }^{b}$

1 a Bucovinian State Medical university, Chernivtsi, 58000, Ukraine,

bucovinian State Medical university, Chernivtsi, 58000, Ukraine

Background: Thyrotoxic cardiomyopathy is a very common chronic condition from the group of metabolic and endocrine cardiomyopathies, develops mainly in young, working-age patients with thyrotoxicosis, often does not acquire the full reverse development in hyperthyroidism compensation, is characterized by chronic, progressive course. Application of Holter monitoring of electrocardiography and assessment of heart rate variability has to objectify and specify metabolic, hemodynamic and autonomic disturbances of Thyrotoxic cardiomyopathy to optimize the complex treatment. Methods: This study was carried out by twice Holter monitoring of electrocardiography and assessment of heart rate variability at the beginning of inpatient treatment and in 1014 days after admission. We analyzed the source and characteristics of rhythm, the heart rate per day and per hour, the frequency and characteristics of arrhythmias, ST-segment depending on the activity, arterial pressure, use of medication. Assessment of heart rate variability included standard time and spectral parameters. Spectral parameters were determined by using of nonparametric method of fast Fourier transformation. Results: Holter monitoring of electrocardiography revealed signs of the hyperdynamic syndrome, morphological heterogeneity of the myocardium and development of cardiosclerosis, diffuse disorders of repolarization because of metabolic disturbances in the heart muscle in all patients. Daily assessment of heart rate variability detected the pronounced increase in sympathetic influence on the cardiac activity regulation, inhibition of the parasympathetic autonomic part. The detected levels of analyzed parameters were substantially differ from the control group. Dynamic supervision identified tendency to improvement of discovered pathologic changes but not up to normal ranges. Conclusion: Diagnoses of the Thyrotoxic cardiomyopathy and its degree of severity should be based on the results of Holter monitoring of electrocardiography and assessment of heart rate variability. Efficiency of pathogenic treatment can be analyzed by dynamics of these settings.

Keywords: Thyrotoxic cardiomyopathy; Holter monitoring of electrocardiography; Assessment of heart rate variability.

\section{Liquid Biopsy - a novel screening method for cancer} ARMAND DURIC

University of Sarajevo, Faculty of Medicine, Sarajevo, 71000, Bosnia and Herzegovina

Background: Tumor biopsies are an essential part of cancer care and patient outcome. A traditional biopsy is a test in which a piece of tissue is removed from a patient for analysis in a laboratory. A pathologist examines the tissue under a microscope, noting the shape, structure, and internal activity of the cells to determine whether the cells are cancerous and, if so, what type of cancer they represent. The identified cell type of the tumor and confirmed a cancer diagnosis are then used to determine whether a patient's tumor contains particular genetic alterations that may make them candidates for targeted therapies. However, tumor biopsies require invasive procedures, including surgery, and some patients may not be candidates because of poor health or because of a tumor's location in the body. In addition, after the initial testing and analyses of biopsy samples, not enough additional tissue may be left for comprehensive molecular profiling, or it may not be of sufficient quality for profiling. Liquid biopsy is a fast, DNA based, noninvasive screening method using the patient's own blood to tests for cancer. Certain fragments of DNA shed by tumors into the bloodstream are used to screen for early-stage cancers, monitor responses to treatment and help explain why some cancers are resistant to therapies. Circulating tumor DNA (ctDNA) liquid biopsy allows us to understand specifically what kind of molecular changes are happening in the tumor in real time, and can provide a dynamic picture of molecular disease changes, suggesting that this approach could also be used to monitor the development of secondary resistance and identify heterogeneous sub-clonal populations of tumor cells developing during the course of treatment. This review will introduce the latest findings on biological features and clinical applications of liquid biopsy and early detection of various cancers. In a word; liquid biopsy is promising to promote precision and very early detection in medicine.

Keywords: Liquid biopsy, circulating tumor DNA cancer, cancer screening method 
77. The development of renal insufficiency after tumor nephrectomy in KBC Rijeka

Matea Hrboka' ,Luka Gorjan', Leo Gulan'', Adrian

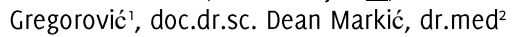

' Faculty of Medicine, University of Rijeka, Rijeka 51000 Croatia, Department of Urology, University Hospital Rijeka, Rijeka, Croatia

Background: Nephrectomy is a surgical procedure to remove kidney and may be performed to treat kidney cancer. The aim of this study is to analyze the global renal function by analyzing creatinine and eGFR before and after surgery. A rise in blood creatinine level is a late marker, observed only with marked damage to functioning nephrons.A better estimation of kidney function is given by calculating the estimated glomerular filtration rate (eGFR). (1) This study is focused on conclusion of ablative procedures, such as nephrectomy, diminish nephron mass and can have a potentially negative impact on renal function. Methods: Data from 120 patients ( 45 female and 75 male) who underwent nephrectomy for kidney cancer in the Department of Urology, University Hospital Rijeka from January 2008 to December 2013 were retrospectively analyzed. Patient with partial nephrectomy were excluded. We measured creatinine serum concentrations, and we estimated glomerular filtration rate (eGFR) preoperatively and postoperatively. Results: The mean preoperative eGFR was 75.07 $\mathrm{ml} / \mathrm{min}$ and mean postoperative eGFR was $52.57 \mathrm{ml} / \mathrm{min}$ $(p<0.0001)$. In the group of patients .65 years old mean preoperative GFR was $67.62 \mathrm{ml} / \mathrm{min}$ and mean postoperative eGFR was $47.48 \mathrm{ml} / \mathrm{min}$ ( $p<0.0001$ ). In the group of patients $<65$ years old mean preoperative GFR was $82.74 \mathrm{ml} / \mathrm{min}$ and mean postoperative eGFR was $58.414 \mathrm{ml} / \mathrm{min}$ ( $\mathrm{p}<0.0001)$. Conclusion: Our data indicate that the eGFR significantly decreased after nephrectomy for kidney cancer. In elderly patients, diminished renal function following nephrectomy was more prominent. Nephrectomy is risk factor for development of chronic kidney disease, especially in the elderly patients. Nephron-sparing procedures should be the treatment of choice in patients with existed renal dysfunction or in patients with recognized risk factors (elderly, proteinuria, diabetes) considering oncologic outcome also.

Keywords: Nephrectomy, Tumor, Creatinine, eGFR, Renal Insufience

78. Decompensation of Chronic Heart Failure due to Cardiac Amyloidosis-Report of a Rare Case

ANDREJ BELANČIĆ ', Marija Krpina ', Luka Vranić ', Ivan Vuksan ', Tamara Hlača Caput ${ }^{2}$, Nikolina Jurjević ${ }^{3}$, Alen Ružić ${ }^{2}$, Luka Zaputović ${ }^{2}$, Teodora Zaninović Jurjević b

School of Medicine, University of Rijeka, Rijeka, Croatia, Department of Cardiovascular Diseases, Clinical Hospital Center Rijeka, School of Medicine, University of Rijeka, Rijeka, Croatia, 3 Emergency medicine institute of the Primorje-Gorski Rotar County, Rijeka, Croatia

Background: Restrictive cardiomyopathy (RC) is a rare form of cardiomyopathy, characterized by diastolic dysfunction and impaired ventricular filling. The most common etiology and archetype of RC is cardiac amyloidosis. Amyloidosis is a rare disease caused by extracellular deposition of insoluble abnormal amyloid fibrils. Acquired serum amyloid $A$ type (AA) amyloidosis triggered by chronic inflammations, involves the heart in about $2 \%$ of cases with systemic AA amyloidosis. Case presentation: We report a case of a 63-year-old female patient with rheumatoid arthritis (RA) and arterial hypertension in her anamnesis and with prior history of myocardial infarction. Patient complained of progressive deterioration in exercise tolerance, occasional chest pain, dyspnea during rest, orthopnea and swollen legs. At admission, blood pressure was $100 / 60 \mathrm{mmHg}$ with heart rate of $92 / \mathrm{min}$. ECG showed low QRS voltages (standard leads) and voltage criteria for LV hypertrophy. On Chest X-ray, bilateral pleural effusion and myopathic configuration of cardiac silhouette were noticed. BNP was $3600 \mathrm{pmol} / \mathrm{L}$ and $\mathrm{TnT} 374 \mathrm{ng} / \mathrm{L}$. Echocardiography (ECHO) revealed thickened right and left ventricle, 'ground glass' appearance of the myocardium, small ventricle cavity size and abnormal diastolic function. Trivial pericardial effusion and moderate mitral regurgitation were also present. All findings revealed the diagnosis of $R C$ most probably due to AA amyloidosis triggered by long-lasting RA, that lead to decompensation of heart failure. Standard therapy for heart failure was introduced. The patient's condition was constantly deteriorating and she was transferred to intensive care unit. Unfortunately, patient developed cardiac shock and died. On autopsy amyloidosis was confirmed by congo red staining and polarized-light microscopy. Conclusion: Due to long-lasting autoimmune disorder in anamnesis, typical clinical X-ray, ECG and ECHO findings cardiac amyloidosis was highly credible. Autopsy has rejected all the possible doubts and confirmed the clinical diagnosis.

Keywords: Amyloidosis; Heart failure; Restrictive cardiomyopathy

\section{Survival and prognostic factors in patients with cirrhosis: a set of 180 patients}

SELMA BOUGHATTAS, Dr. Rania Hefaiedh

Background: Cirrhosis is a chronic liver disease whose evolution is characterized by the occurrence of serious complications. The study of prognostic factors in this disease has been the subject of renewed interest due to advances in care. It has therefore become important for the clinician to estimate the survival of the patient to adapt its management. Many prognostic scores have been developed by the Western countries. By contrast, studies on the Tunisian cirrhotic are few. Aim: to determine survival in Tunisian cirrhotic and to identify the specific prognostic factors in this population. Methods: Retrospective descriptive study involving all cirrhotic patients managed in Gastroenterology department of Mongi Slim hospital during the period from January 2009 to December 2013 was conducted. Univariate and multivariate studies was performed. Results: One hundred and eighty patients were included from whom 99 men. The average age was 59 years. Viral cirrhosis was noted in $59 \%$ of cases, it was respectively post viral $B$ and $C$ in $25 \%$ and $33 \%$. In 120 patients (66\%), cirrhosis was inaugurated by a complication. Overall mortality was $44 \%$. It was of the order of $3 \%$ the first year and $9 \%$ the second year. The prognostic study in univariate analysis of 34 epidemiological, clinical, biological and therapeutic variables, identified 12 predictors directly associated with deaths: male gender $(p=0.014)$, tobacco consumption $(p=0.02)$, viral etiology $(p=0.014)$, particularly hepatitis $B(P=0.015)$, AST level $(p=0.003)$. PAL level $(p=0.03)$, total bilirubin level $(p=0.009)$, creatinin level $(p=0.013)$, natriuresis $(p=0.007)$, alpha foetoprotein level $(p=0.023)$, the ascitic fluid infection $(p=0.056)$ and hepatocellular carcinoma $(p=0.0001)$. In multivariate analysis, two variables emerged: the presence of hepatocellular carcinoma and serum total bilirubin. Conclusion: Our study showed that cirrhosis was associated with high mortality and poor overall survival. Thus, through our results, the occurrence of complications during cirrhosis worse prognosis.

Keywords: Cirrhosis-Survival- Prognosis

\section{Poster session II:}

\section{Salivary gland tumor children}

DARKO KOTROMANOVIĆ ${ }^{1}$,Tin Prpića, Darjan Svetinović ${ }^{1}$ Sonja Kotromanovićc ${ }^{, Z ̌ e l j k o ~ D u m a n c ̌ i c ́ 1, ~ I v a n a ~ T o v i l o ', ~}$ Barbara Likar1,Melita Peček', Željko Kotromanović ${ }^{2}$ ' Faculty of Medicine University of Osijek, Osijek, 31000, Croatia, University of Osijek, Osijek, 31000, Croatia

Background: Salivary gland tumors in childhood are not common, the classification is complex and substantially different from those of adults. The most common tumors are hemangioms whose etiopathogenesis is not known. Aim: The aim of this study was to make a difference between benign and malignant tumors of salivary glands in childhood, because they can be hard to identify. Methods: The ultrasound examination has shown hypoechoic node, which was about $2 \mathrm{~cm}$ in diameter, in the right parotid gland. A magnetic resonance imaged a node of the same localization and size. We also did the fine needle aspiration biopsy and saw a benign tumor of parenchymatous tissue (pleomorphic adenoma Results: The treatment consisted of parotidectomia superficialis I.dex. (with the display and preservation of the facia nerve). Conclusion: Salivary gland tumors in childhood are rare. 
They differ in incidence, appearance, and behaviour in relationship with those of adults. They make about $0,3 \%$ of all human head and neck tumors. They make less than $10 \%$ of all child head and neck tumors. They can be benign and malignant. Epithelial tumors make $3-22 \%$. $88,5 \%$ of tumors are tumors of the major salivary glands and $11,5 \%$ of the major salivary gland tumors are malignant. The incidence is $1-2$ of 100000 . There are various published results which confirm the rarety of salivary gland tumors during childhood: Luna- 6 centres- 2823 patients - 324 children under 16, Castro- 2135 patients- 38 children aged $5-16$, Orvidas 118 children during 27 years and Rodriguez- 11 children during 12 years.

Keywords: pleomorphic adenoma, parotid gland, radiotherapy

81. Physical and mental functioning in children with primary headache.

Natalia Kovtyuk MD, PhD 1 ,Poberezhnyi Andrii, student?, Yurii Nechytaylo MD, Professor ${ }^{2}$

7 Associate professor. Department of pediatrics, neonatology and perinatal medicine. Bucovinian State Medical University, Chernivtsy, Ukraine, 2 Head of department of pediatrics, neonatology and perinatal medicine. Bucovinian State Medical University, Chernivtsy, Ukraine, 3 Student of 1-rd year, medical faculty, Bucovinian State Medical University

Background: Headache disorders are one of the most common and disabling pain conditions in children. Frequent headache in children was associated with negative psychosocial impact such as school absence, higher levels of emotional problems, in particular anxiety and depression, as well as other somatic complaints and lower levels of quality of life ( $\mathrm{Q} \mathrm{OL})$. Aim: The goal of prospective study was to establish influence of primary headache on children's health-related quality of life. Methods: We examined 98 children (age 10-17 years). All participants were assessed using validated instruments for measuring physical (physical health summery - PHS) and mental (mental health summery - MHS) functioning. We used generic questionnaire of $\mathrm{QOL}$ with Children Form health surveys (CF). We were carried out the medical examination, questionnaire with refinement social, anamnesis, and other features. We were looking on a direction, force and significance of correlation. Results: Quality of life is recognized as an important outcome of children health. The main parts of QoL estimation include physical and mental functioning summary and overall quality of life summary. The difference in MHS between healthy (77.8 \pm 13.0$)$ and PHA (73.1 \pm 11.9$)$ children was significant $(p<0.05)$. The biggest difference between healthy $(84.6 \pm 12.9)$ and headache $(76.7 \pm 14.1, p<0.05)$ children was seen in PHS. We found out some difference in gender depending child self-report. The girl's data comparing with boy's had wider range (76.2 vs 61.4) and general higher assessment (77.4 vs 76.1) of their physical functioning index. Children with headache reported a worse overall index of QoL (QLS) (73.9 \pm 9.4$)$ as compared to age-related healthy individuals $(78.8 \pm 10.1, p<0.05)$. Thus generic questionnaire CF is particularly useful when comparing health-related quality of life of subpopulations with different diseases with intermittent character, like headache, with a healthy control group. Conclusion: Primary headache in children and adolescents is a common problem which influenced health-related quality of life. Physicians need more knowledge about QoL indexes and their associated factors in children.

Keywords: headache, quality of life, children.

82. Features of nail bed capillaries in children with chronic gastroduodenitis

TETIANA MIKHEEVA

Bukovinian State Medical University, Ukraine

Background: Today we know that pathogenesis of chronic gastroduodenal diseases is complex and multifaceted. Proved that microcirculatory changes can determined gastroduodenitis option and they are quite early marker of the degree of target-organ damage. Methods: We examined 80 children who were hospitalized in the gastroenterology department of Children's
Clinical Hospital, Chernivtsi. Children were divided into two groups: 1 group (40 children) included children with chronic gastroduodenitis, 2 group (40 children) - with functional gastroduodenal pathology. Average age amounted 13,6 $\pm 0,12$ years. We used the following methods: clinical, anthropometric, laboratory and statistical tools. For assessment of microcirculation children spent capillary microscopy of nail bed with a digital microscope with $400 X$ fold magnification. Saved photos were evaluated on a portable computer with a special program. Results: For evaluation of the microcirculation all children conducted biomicroscopy of capillary of nail bed. We found the following changes: pathological tortuosity of capillaries, spasm of capillaries, slowing blood flow, the phenomenon of "sludge", abnormal form of capillaries, changes of caliber, reducing the total number of capillaries per unit area, the availability of additional anastomoses. All changes were divided into: perivascular changes, vascular changes (venules, arterioles and capillaries), intravascular changes (venules, arterioles and capillaries). Most children with chronic gastroduodenitis had a standard U- shaped arrangement of parallel of nail bed capillaries. Most children with functional gastroduodenal pathology also had a standard U- shaped with parallel arrangement of capillaries of nail bed, pathological tortuosity of capillaries observed in six children (20\%), spasm of capillaries - in four children (13\%), slowing blood flow was observed in one child $(3 \%)$, the phenomenon of "sludge" - in one child $(3 \%)$. Conclusion: Thus, the above changes in the nail bed capillaries in school age children with chronic gastroduodenal pathology may be early signs of diseases of the stomach and duodenum.

Keywords: school-age children, chronic gastroduodenitis, microcirculation, microscopy of nail bed.

\section{FEATURES OF ENDOTHELIAL FUNCTION IN SCHOOL AGE CHILDREN WITH ARTERIAL HYPERTENSION NECHYTAILO DMYTRO}

' Bukovinian state medical University, Ukraine

Background: Endothelial dysfunction is characterized by impaired circulation, shortness deliver blood and oxygen to the tissues, which in turn can cause serious diseases of the cardiovascular system. Methods: The study involved 80 school-age children of Chernivtsi region. Ukraine. 1-st group (40 children) were children with elevated blood pressure who were treated in the pediatric department of Children's Clinical Hospital, Chernivtsi; 2-nd group (40 children) - healthy children. Average age was 14.3 years. To determine the level of blood pressure children performed tonometry. Endothelial function in children was evaluated during biomicroscopy of nail bed with a digital USB-microscope with a maximum increase $400 \mathrm{X}$ using occlusive and temperature tests. Results: When performing occlusive and temperature tests in children from 1 group was revealed the following changes: local spasm of capillaries observed in 26 children with pre-hypertension in 9 children with hypertension; slowing blood flow in venules was in 10 children pre-hypertension in 8 children with hypertension. In 14 hypertensive children observed "sludge-phenomenon." In 6 children we saw a picture of reducing of number of capillaries per unit area. The temperature test, on the contrary, helped reduce local spasm and "sludge" in the capillaries in 14 children with hypertension. Children from the second group during occlusive and temperature tests showed next results: local spasm of capillaries in 8 children, slowing blood flow in venules - 5 children, the phenomenon of "sludge" - 7 children. After heating the tests local spasm of capillaries was detected in 2 children. Conclusion: Children with elevated blood pressure during occlusive and temperature tests significantly more often shows signs of endothelial dysfunction than healthy children, which is understandable consequence of autonomic disregulation which in turn is accompanied by disorder of microcirculation at all levels, including capillaries .

Keywords: school age children, endothelial dysfunction, blood pressure. 


\section{The incidence of hepatitis B and C infection among opiate drug users in Canton Sarajevo \\ Šljivo $A^{1}$, Dedić $V^{\prime}$, Čvorak $0^{1}$, Delić $N^{\prime}$, Mlačo N', Mulać $A^{1}$ Kulo $A^{\prime}$ \\ ${ }^{1}$ Medical faculty of the University of Sarajevo, Sarajevo, 71000, BiH}

Background: Hepatitis C virus (HCV) and hepatitis B virus (HBV) transmission among people who inject drugs remains a challenging public health problem. Sharing of needles, syringes and other injecting equipment is the most frequent way hepatitis virus is transmitted from an infected person to an uninfected person Methods: This is a cross-sectional study on drug dependence patients who have been hospitalized in "Public Institutions of Hospital for addict disease of Canton Sarajevo" for period from 01.10.2015. to 01.10.2016. Subjects $(n=129)$ were divided into two groups: Group I: Control group $(n=55)$ subjects consuming opiate by inhalation and Group II: Investigate group $(n=74)$ subjects consuming heroin by injection. Results: The Group I (inhalation) has $81 \%$ male participants and the Group II (injection) has $82,4 \%$. Mean age of both groups was between 30 and 40 years. In the Group I $21,9 \%$ were HCV positive and in the Group II 40,5\% participants were HCV positive. In the Group I 3,6\% participants were HBV positive and in the Group II 14,8\% HBV were positive. Also, in the group II were 7 cases or $9,4 \% \mathrm{HCV}$ and HBV positive. Conclusion: Consuming opiate by injection than by inhalation showed significant $(p<0,05)$ risk for hepatitis $B$ and hepatitis C infections.

Keywords: drug users, hepatitis C, hepatitis B.

85.

KNOWLEDGE OF MENTAL HEALTH FIRST AID AND
DISCRIMINATION AGAINST PEOPLE WITH MENTAL ILLNESS
STUDY
NERINGA CENAITE', MARIJA SIAUCIUNAITE2, Arunas
Germanavicius ProfesSor, MD, PhD33
' Faculty of Medicine, Vilnius University, Lithuania, 2 Clinic of
Psychiatry, Faculty of Medicine, Vilnius University, Lithuania

Background: Discrimination against people with mental health problems (MHP) is prevalent, though people are likely to have a contact with mental health (MH) crises. MH first aid (MHFA) teaches society to identify MHP. According to studies negative attitudes towards MHP had decreased after MHFA trainings. Our aim was to compare knowledge of MHFA and an assumption of discrimination against people with MHP in Lithuania. Methods: "Australian National Survey of MH Literacy and Stigma" was adapted to an anonymous internet survey. The questionnaire contained: demographic information; case vignettes (concerning depression, depression with suicidal thoughts, early schizophrenia, and chronic schizophrenia) - one of them was obtained randomly to every participant; identification of MHP and an opinion on whether a vignette person would be discriminated in society was tested. MHFA knowledge was evaluated by the ALGEE algorithm, maximum score - 5. Analysis used SPSS. Results were considered statistically significant when $p<0.05$. Results: 182 people took part in the survey during April 2016. 175 were involved in the final analysis. The age of respondents 18-59; male $24.6 \%$, female $75.4 \%$. The average evaluation of ALGEE was $1.84 \pm 0.89$, distribution by gender: female $1.96 \pm 0.89$, male $1.60 \pm 0.82$ $(p=0.021)$. ALGEE scores by identification of vignettes: correct identification $(n=136) 1.95 \pm 0.88$, wrong $(n=39) 1.62 \pm 0.88(p=0.04)$. No statistically significant difference between ALCEE score groups by assumption of discrimination occurred $(p=0.829)$. Participants who had vignettes with schizophrenia were more likely to assume that a vignette person would be discriminated and those who had vignettes with depression assumed contrariwise $(p=0.003)$. Conclusion: People of Lithuania have low knowledge regarding MHFA and female showed better results. The average ALCEE score was 2 of 5 . There were no association between ALGEE knowledge and opinion on whether people with MHP would be discriminated. People assumed that a person with schizophrenia would be discriminated in society and someone with depression would not.

Keywords: Discrimination, mental health first aid, mental health
86. Tactile hallucinations at the patients suffering from depression - case study

Andrijana Mišković ', Krešimir Šantić ', Dunja Degmečić 1,2 $\checkmark$ Faculty of Medicine Osijek, Josip Juraj Strossmayer University of osijek, Osijek, Croatia, 2 University Hospital Center Osijek, University Department of Psychiatry, Osijek

Background: Hallucinations were commonly treated as symptoms associated solely with patients suffering from schizophrenia, rarely they were associated as an important symptom related to some other psychic disorders. Tactile or haptic hallucinations in the skins touch sense can be diverse, sensed as ranging from ant crawling to insects crawling, poking, stitches with sharp objects, skin burns or even electrifying or touching. Case presentation: A 33 years old female patient whose initial diagnosis is $F_{33}$ and psychically is treated by a psychiatrist for many years had the first hospital admission in 2003 due to suicidal attempt. The patient had repeatedly shown suicidal behavior form and because of depressive decompensation on several occasions was hospitalized at The Department of Psychiatry, KBC Osijek and also in othe institutions. During the last few years, she has begun to consume alcohol with the inability of self-control and establishing of abstinence. Last outpatient therapy was in September 2016, when the doctor had noticed that the patient had lowered mood, lethargy, restlessness, tension, anger, focusing on somatic disorganized thinking, blocks of thought and fear. The patient was diagnosed with F33.3 Major depressive disorder, recurrent episode, with psychotic features. At the reception the patient mentioned existing intense tactile sensory deceptions that interfere with her daily activities. During hospitalization, the patient was treated with the combination of antipsychotics and antidepressants. It is also important to note that the patient uses hormone substitution therapy due to hypothyroidism. Conclusion: With this case study we wanted to point out the importance of observing each patient individually with their physical and mental conditions. We also wanted to point out the wide range of possible appearance of psychopathology in patients with depressive disorders. The case is presented with the approval of the patient.

Keywords: hallucinations, tactile hallucinations, depression, symptom.

87. The Role of Consultative Liaison Psychiatry in elderly in Šumadija district

Ljiljana Arsenijević1, Jelena Milutinović ${ }^{2}$, Branimir Radmanović1,2

7 Faculty of Medical Sciences, University of Kragujevac, Kragujevac, 34000 , Serbia, 2 Department of Psychiatry, Clinical Centre Kragujevac, Kragujevac, 34000 , Serbia

Background: Consultative Liaison psychiatry represents knowledge and skills that are used in evaluation and treatment of emotional and behavioral condition of patients that are treated at non psychiatric clinics and departments. Geriatric population in consultation-Liaison psychiatry is challenge due to the high percentage of somatic comorbidity and biological changes that occur with aging and affects choice of therapy. Aims of this study were extraction of the most common psychiatric diagnoses and symptoms and the analysis of the prescribed therapies compared to leading recommendations. Methods: A descriptive, retrospective cross-sectional study was conducted in order to determine the most common psychiatric diagnoses, the most commonly used therapies in their treatment and the compatibility of selected therapy with national and international guidelines. The study was conducted in Gerontology Center in Kragujevac. The study includes all the patients examined by the psychiatrists for the time period from the 1st of September, 2014 until the 1st of September, 2015. Results: The study includes 179 patients, with average age of $73.76 \pm 12.48$. The most common psychiatric diagnosis are dementia and depression disorders while the most commonly used medicaments are lorazepam, risperidon and trazodon. In addition, around $45.18 \%$ of the patents from Gerontology Center uses some sort of benzodiazepines lorazepam, bromazepam, diazepam. The correlation is statistically significant between patients' age and dementia and depression 
disorders. Conclusion: Dementia, depression and psychosis are the most common reasons for consulting a psychiatrist. The prescribed therapy is according to the actual recommendations. High (and relatively disturbing) percentage of patients who were administered benzodiazepines indicates a need for further analysis and research in this direction, while the use of alternative therapies (Trazodone) in this case is encouraging.

Keywords: Consultation-Liaison psychiatry, gerontology centers, elderly.

\section{THE LEVEL OF ANXIETY IN SCHOOL AGE CHILDREN WITH ARTERIAL HYPERTENSION NECHYTAILO TETIANA' \\ $\checkmark$ Chernivtsi national University, Ukraine}

Background: In the last time cardiovascular diseases have one of the leading positions in the world among children deseases. Typically, hypertension in children is the result of autonomic dysfunction, which in turn is closely linked to the psychological state of the child and the level of anxiety. Methods: We examined 30 school age children (average age 14.7 years) from. Chernivtsicity. The children were divided into two groups: basic and control. The basic group (20 children) includes children with diagnosed arterial hypertension. In the control group (10 children) were healthy children. To assess the level of anxiety was used scale of Spielberger-Hanin. Results: The average level of situational anxiety in children of the basic group was 35.4 points, which is a moderate level of anxiety. The average level of anxiety in children of the control group was low (27.6 points). A similar pattern was observed when assessing the level of personal anxiety. Average level of personal anxiety in children from basic group was 42.6 points, which is actually border with high level of anxiety. In addition, $4(25 \%)$ children personal anxiety level was above 40 points, that mean high index. In the control group the average level of personal anxiety was low and had 28.7 points. Conclusion: Thus school age children with arterial hypertension observed significantly higher levels of both personal and situational anxiety that have negative impact on prognosis of the disease course. In addition, for more effective treatment of hypertension in school age children, special attention should be given to methods of reducing anxiety in these children.

Keywords: school age children, arterial hypertension, personal anxiety level, situational anxiety level.

89. PHARMACOTHERAPY' TRENDS IN PRESCRIBING THERAPY FOR BEHAVIORAL AND PSYCHOLOGICAL SYMPTOMS OF DEMENTIA, PATIENTS WITH ALZHEIMER'S TYPE DEMENTIA

Jelena Milutinović', Ljiljana Arsenijević2, Branimir Radmanović1,2

1 Faculty of Medical Sciences, University of Kragujevac, Kragujevac, 34000, Serbia, 2 Department of Psychiatry, Clinical Centre Kragujevac, Kragujevac, 34000, Serbia

Background: Behavioral and psychological symptoms of dementia, affects more than $80 \%$ of Alzheimer's disease patients' and it is one of the most common reasons for early institutionalization and increasing the total cost of treatment. The real problem occurs during the beginning of pharmacotherapy due to biological aging, which involves changes in pharmacodynamics and pharmacokinetics, sensory changes, structural changes in organs, neuronal loss; the psychological problem and social problem. Analysis of pharmacotherapy and prescribed medications used in the treatment of behavioral and psychological symptoms of dementia in the territory of the Šumadija region and comparing the results obtained with the current guides and recommendations. The second objective relates to the existence of differences between the institutional pharmacotherapy of patients cared for and those who are outpatients receiving care. Methods: We performed a descriptive, retrospective cross-sectional study. The study was conducted at the Clinical Center and the Gerontology Centre in Kragujevac. The study included patients examined by psychiatrists in the period from 01.09.2014. until
01.11.2015. year. Results: The study included 115 patients, mean age $79.25 \pm 6.991$ years. Institutional patients was 62 , while the ambulatory patients cared for was 53 . Of all the institutional cared for patients, $43.55 \%$ is on antidementia therapy, while this number is higher in patients whom are ambulatory cared for and that is $56.6 \%$. The most frequently prescribed included donepezil, memantine and rivastigmine. Conclusion: The therapy, which is prescribed to people suffering from dementia for behavioral and psychological symptoms of dementia is in line with the recommendations of national and international conductors. Institutional disposed patients have lower (personalized) doses of drugs than patients who are treated ambulatory.

Keywords: Alzheimer's dementia; behavioral and psychological symptoms; pharmacotherapy.

\section{A Comparison of Attitudes Towards Alcohol Among} Students of Different Montenegrin Faculties

VUKČEVIĆ BATRIĆ'; Vukčević Nemanja ${ }^{2}$

1 University of Montenegro, Medical Faculty Podgorica, ${ }^{2}$ University of Montenegro, Medical Faculty Podgorica

Background: Alcohol consumption is a widespread social problem in Montenegro and the world, especially among youth. Researching alcohol consumption among youth is of considerable practical importance when it comes to planning activities in order to solve this problem. Our aim was to determine the prevalence and compare the alcohol consumption among the students of the Faculty of Medicine and the Faculty of Political Science of the University of Montenegro. Methods: The research was performed between October and November 2015. The Alcohol Use Disorder Identification Test, created by the World Health Organization, was used. The score in this test was utilized to determine hazardous drinking. Results: The research included 39 students of the Faculty of Medicine (of which $48 \%$ were female and $52 \%$ were male) and 38 students of the Faculty of Political Science (of which $95 \%$ were female and $5 \%$ were male). In both faculties, $74 \%$ participants were alcohol drinkers, with a difference in sex structure: the drinkers from the Faculty of Medicine had the structure of $41 \%$ female and $59 \%$ male, while the drinkers from the Faculty of Political Science had the structure of $7 \%$ male and $93 \%$ female. Depending on the score, $59 \%$ drinkers from the Faculty of Medicine were labeled as hazardous (mostly male), as well as $18 \%$ drinkers from the Faculty of Political Science (of which $40 \%$ were male). Conclusion: It is necessary to perform suitable preventive measures in order to change drinking behavior among students.

Keywords: alcohol, students, AUDIT, Montenegro

91. The New Potential Topical Activated Carbon Gel to Prevent Green Tobacco Sickness (GTS) in Tobacco Farmworkers LIDYA PERTIWI SUHANDOKO', Siti Ermawati', Nuzula Fikrin Nabila'

' Faculty of Medicine University of Airlangga, Surabaya, 60301, Indonesia

Background: Green Tobacco Sickness (GTS) is a disease that occurs in tobacco farmers as a result of nicotine intoxication through skin contact with the tobacco leaves ${ }^{1,4.5}$. GTS incidences is high; in Brazil 2013 there are 107 incidences among 130 group sample3. GTS symptoms are dizziness, headaches and nausea or vomiting, but usually can occur in more severe way so can lead to dehydration and require emergency care4. Current method in preventing GTS such as using gloves, boots, and washing hands are not effective enough ${ }^{4}$. Therefore, a new effectively cheap material is needed to prevent the GTS incidence. Methods: Through literature review methods selection including all of the keywords and other related terms. Sources of data obtained (Randomized Control Trial, Systematic Review-Meta Analysis and conclusions of research) to the level of evidence-based hierarchy between $1 \mathrm{~A}$ to $2 \mathrm{C}$. Earlier publication and original research were highly regarded then a descriptive analysis is performed and a new idea is synthesized. Results: The adsorbent activated carbon gel is widely used and proven capable of absorbing nicotine. Activated carbon gel can be 
produced from various raw materials that contain much carbon using steam activation method and then broken down into smaller sized powder to be formulated into a gel form. Other materials used are hydroxypropyl methylcellulose and carbomer as a base gel, methyl paraben as a preservative, and propylene glycol as a solvent. Through topical administration before wearing gloves and used without gloves on the skin surface harvesters Conclusion: From this study, it is effective to use activated carbon gel to prevent the GTS among tobacco farmworkers as it can prevent nicotine enter the skin and result in increasing the safety and productivity in the agro industrial field of tobacco farmworkers.

Keywords: green tobacco sickness, activated carbon, agroindustrial medicine

\author{
Ionizing radiation is a hazard: Is your imaging request \\ justified? \\ Ceorgiana Farrugia
}

Background: During the past few years, the extent of ionizing radiation exposure amongst patients has increased dramatically. It is often the case that non-radiologists request examinations involving ionizing radiation. Moreover, consultations with a radiologist prior to submitting such imaging requests is not required in most hospitals, with the exception of highly specialized procedures such as CT scanning, MRI, RNI and Angiography. Previous European studies have shown that a significant portion of various radiological examinations being requested by medical doctors were either inappropriate or unneccesary. Methods: This study aimed to determine whether the patients' exposure to ionizing radiation is justly indicated for diagnostic or clinical management purposes, according to standards defined in the European Commission (Radiation Protection 118) Referral Guidelines for Imaging. This audit involved the investigation of 750 imaging request forms of adult patients who have had an ionizing general imaging investigation at the state general hospital between January 2016 to June 2016. The author tabulated brief clinical details from imaging request forms, namely 44 head and neck x-rays, 98 spine x-rays, as well as 420 musculoskeletal system, 140 cardiothoracic and 48 gastrointestinal system $x$-rays. This information was then compared to standards defined in the European Commission Referral Guidelines for Imaging to fulfill the objective outlined earlier. Data analysis was carried out by means of simple descriptive statistics. Results: Out of a total of 750 imaging requests, $494(66 \%)$ were considered to be indicated for clinical or diagnostic purposes. The majority of non-indicated request forms belonged to the head and neck as well as spine imaging categories. Conclusion: Conclusions from the data analysis indicated that referring physicians possess intermediate knowledge with regards to justification of imaging criteria. Following short lectures on this topic, patients are likely to benefit from the reduction of unnecessary exposure to ionizing radiation.

Keywords: radiation, justification, imaging, request, indication.

93. Prophylactic Peritoneal Incision: Prevention of Lymphoceles after Retropubic Radical Prostatectomy Accompanied by Pelvic Lymphadenectomy

ANDREJ BELANČIĆ ', Marija Krpina ', Kristian Krpina ${ }^{2}$

' Faculty of Medicine, University of Rijeka, Croatia, ${ }^{2}$ Department of Urology, Clinical Hospital Centre Rijeka, Croatia

Background: High percentage of patients with prostate cancer undergo and benefit from retropubic radical prostatectomy (RRP). In some cases, RRP is accompanied by bilateral pelvic lymph node dissection (PLND). The most common complication after PNLD are lymphoceles, which are present in almost $50 \%$ of patients. However, only $3-9 \%$ of those are symptomatic and/or require intervention. Aim of our study was to determine the effect of so called "prophylactic peritoneal incision" (PPI) on lymphocele incidence. Methods: This is a retrospective study of patients who underwent PPI after RRP and PNLD performed by a single-surgeon at the Department of Urology, CHC Rijeka from 2014 to 2016. The
$\mathrm{PPI}$ is based on incision in peritoneal layer close to the internal iliac artery region with special emphasis on defining the course of ureter and avoiding it within secure distance. The same procedure was performed bilaterally, upon completion of the urethrovesical anastomosis and prior to insertion of a pelvic drain. We have analyzed hospital and ambulatory medical charts of investigated group. Reported symptoms, such as abdominal pain, leg pain, fever and lower limb edema, were evaluated. Ultrasound examination of the pelvis, looking specifically for lymphoceles, was performed one month after the surgery. Results: The investigated group constituted 36 patients. Median age was 68 years (range 52-77 years). Mean preoperative PSA value was 37,78 $\mathrm{ng} / \mathrm{mL}$ (range 2,33-243 ng/mL). Preoperative Gleason scores were: 6 $(12,9 \%), 7(48,4 \%), 8(16,1 \%), 9(12,9 \%), 10$ ( $9.7 \%)$. Mean number of removed lymph nodes was 20 (range 8-43). On first routine ambulatory check-up, no lymphoceles were detected on ultrasound examination in investigated group. In medical charts we have found inconsistently reported symptoms, so we will not make any conclusions on PPI impact on symptom rate. Conclusion: PPI leads to prevention of lymphocele formation. More objective diagnostic options (CT scans) should be considered in further investigation.

Keywords: Prostate cancer; Prophylactic peritoneal incision; Lymphocele

\section{Septic arthritis after wound dehiscence in total knee replacement}

Jošt Janša ${ }^{{ }^{*}}, \mathrm{MD}$, Jernej Bergant ${ }^{2}, \mathrm{MD}$

1 General Hospital Jesenice, Slovenia, ${ }^{2}$ University Clinical Center Ljubljana, Slovenia

Background: Septic arthritis can be defined as the invasion of a joint by an infectious agent. The incidence of septic arthritis varies from 2 to 10 per 100,000 in the general population ${ }^{1}$. In one study examining 6210 patients after total knee replacement overall early deep infection rate for patients undergoing a primary knee replacement was $0.39 \%$, whereas the rate for patients undergoing a revision knee replacement was $0.97 \%$. Case presentation: 60 year old patient admitted to the hospital for total knee replacement. She got i.v. Eliquis, Ugurol, Cefamezin, Cerson postsurgery. The day after surgery she falls on the operated knee and the wound broke open. She had her wound re-sutured. Her wound was healing properly and hematoma regressed. One month after the surgery she is re-admitted to the hospital due to severe serohemorrhagic effusion. Her wound had necrectomy and revision done. After the results of antibiogram, she was treated with Vankomicin. After being discharged for the second time, she gets Linezolid and Rifampicin for 4 weeks and after 4 weeks Rifampicin and Primotren for 3 months. After 3 months redness around the wound persists and parameters of inflammation are still high. We decide for explantation of the knee replacement. Conclusion: Despite incidence of septic arthritis in total knee replacement lower than $1 \%$, it is a serious burden for patients and doctors. There may be different causes for the infection. Our case showed a preventable cause - falling and dehiscence of wound. More preventive measures for falling in days after surgery should be discussed.

Keywords: septic arthritis; total knee replacement; infection; dehiscence.

95. Gender difference of posterior condylar offset ratio among Croatian population

LEO GULAN $1^{*}$, Luka Gorjan 1, Matea Hrboka ', Adrian Gregorović 1

' Faculty of Medicine University of Rijeka, 51000 Rijeka, Croatia

Background: The geometry of the knee shows variations between genders and various ethnic groups. One of the parameters which participates in knee geometry is the posterior condylar offset (PCO) which is defined as posterior condyle projecting posteriorly to the posterior femoral shaft (1). It is recognized as an important factor, which should be reconstructed as precise as possible 
during total knee arthroplasty, because it has a great influence on postoperative knee stability range of motion and patients satisfaction. One of the methods to describe and quantify PCO is the posterior condylar offset ratio (PCOR). The aim of this study is to determine and compare PCOR among Croatian male and female population. Methods: The PCOR of 200 random knees (105 male, 95 female) were obtained from database of Clinic for Orthopaedic Surgery Lovran. Knees with mild or severe osteoarthritis were excluded. On true lateral plain radiograph the first line was drawn as a tangent of posterior cortex of femoral shaft. The second line was parallel with the first, passing through anterior cortex. The third line was drawn parallel to the first line but passed through the most posterior point of the femoral condyles, representing the posterior condylar tangent line. PCOR is defined as a ratio of the distance between the first and the third line divided by the distance between the second and the third line. Results: The mean ratio for female PCOR was 0,468 (SD 0,033) and for male 0,443 (SD 0,029 ). By comparing these two groups we can conclude that the female PCOR in Croatian population is significantly larger than the male ones $(P<0,001)$. Conclusion: PCOR significantly differs between genders among Croatian population. This finding should be taken into account during selection of prosthesis when performing total knee arthroplasty and in postoperative radiographic evaluation.

Keywords: Knee, gender, posterior condylar offset ratio, arthroplasty

96. Hypotension Status of Patients Undergoing Caesar Surgery with Spinal Anesthesia and the Correlation with Babies' Birth Weight in Ciptomangunkusumo General Hospital

JENNI PRATITA' ${ }^{1}$, Alfan Mahdi Nugroho ${ }^{2}$

7 Faculty of Medicine, Universitas Indonesia, Jakarta, 13350, Indonesia, 2 Ciptomangunkusumo General Hospital, Faculty of Medicine, Universitas Indonesia, Jakarta, 13350, Indonesia

Background: Hypotension is a common complication among patients undergoing caesar surgery with spinal anesthesia. Hypotension could cause maternal and fetal death, both of which are major problems in Indonesia. However, the number of studies on this topic in Indonesia is still very limited. Therefore, this study aimed to find out the socio demographic and clinical characteristics of patients undergoing emergency Caesar surgery with spinal anesthesia in Ciptomangunkusumo General Hospital (type of fluid given, age, concurring illness, injection site, dosage of anesthetic solution, height, and hypotension status) and the correlation between hypotension status and the babies' birth weight. Methods: This study was a cross-sectional study with 107 subjects acquired through fulfillment of study criteria and consecutive sampling. Subjects were patients undergoing emergency Caesar surgery in emergency department of Ciptomangunkusumo General Hospital. Data was obtained from subjects' medical records. The definition of hypotension used in this study was a decrease of systolic pressure below $80 \%$ baseline pressure measured before the surgery. Results: Hypotension was found in $24.3 \%$ subjects. No statistically significant correlation was found between the babies' birth weight and the hypotension status $(p=0,692)$, but the mean birth weight of babies from the hypotension group was higher than non-hypotension group $(2512,69 \pm 886,993$ g vs. $2251,65 \pm 721,723$ g). Conclusion: The prevalence of hypotension in this study was rather low compared to other studies, presumably due to difference in definition of hypotension, poor intra operational blood pressure recordings, and differences in subjects' sensitivity to anesthetic agent and characteristics. Majority of the subjects, based on the characteristics stated before, did not have risk factors for hypotension, except that most subjects received crystalloid instead of colloid. Although there was no statistically significant correlation, but the mean birth weight of babies from hypotension group was higher, which is in accordance with previous studies.

Keywords: Hypotension, caesar, spinal anesthesia

\section{Rare Manifestation of Lymphedema Tarda \\ JERNEJ BERGANT ${ }^{*}$, MD, Tina Sirc ${ }^{2}$, MD \\ ${ }^{1}$ University clinical centre Ljubljana, Slovenia, ${ }^{2}$ General hospital \\ lesenice, Slovenia}

Background: Lymphedema is defined as the abnormal accumulation of interstitial fluid and fibroadipose tissues. Primary lymphedema is rare, especially when unilateral. It is categorized by age: congenital lymphedema (first years of life), lymphedema praecox (puberty to 35 years of age), and lymphedema tarda ( $>35$ years). Secondary lymphedema occurs following trauma, surgery, radiotherapy, immobility or infection. (1) Case presentation: 37 y.o. lady presented with swelling of 2nd and 3rd MTP joints of right hand with unremarkable history. Function of the hand was normal. Complete blood count, CRP, SR, uric acid, antistreptolysin 0 titer, waaler-rose and latex RF tests were negative. Diclofenac $2 \times 1$ and local cooling didn't help. Hand X-ray showed moderate early degenerative changes on all small joints. Infectious and rheumatic pathologies were excluded. After 4 months she returned with paraesthesia in 4th and 5 th finger of right hand. EMG showed early signs of $\mathrm{n}$. ulnaris compression. A year since onset of disease she presented with swollen, stiff, bluish, painless hand and wrist, resembling lymphedema. Complete blood count, SR, CRP, tumour markers were normal. We referred her to doppler sonography of arm, chest x-ray and clinic for breast diseases where mammography and breast sonography were performed. All the investigations were normal. Conclusion: Before making the diagnosis of primary lymphedema we have to exclude secondary causes. Among the primary lymphedemas, lymphedema tarda is the rarest. It is uncommon in the upper limb and most of documented cases have involved the entire arm. Unilateral localized hand and wrist lymphedema is even rarer. $(1,2)$ First-line therapy is physical therapy including compression (minimum $40 \mathrm{~mm} \mathrm{Hg}$ ), layered bandaging, physical exercise, massage, pneumatic pumps and elevation. (3) It is important to emphasize that prompt therapy is key to minimize irreversible fibrosclerotic changes within tissues, which would lead to progressive hand dysfunction.

Keywords: lymphedema, tarda, swelling, hand.

\section{Goldston Syndrome: A Case Report of Dandy-Walker} Malformation With Polycystic Kidney In Antenatal Presentation Khaerani AS', Afidjati $H^{1}$, Akbar $F^{1}$, Sungkar ${ }^{2}$

FFaculty of Medicine, Universitas Indonesia, 2Department of obstetric and Gynaecology, Ciptomangukusumo Hospital, Faculty of Medicine, Universitas Indonesia

Background: Coldston syndrome is condition of Dandy-Walker Malformation (DWM) associated with polcystic kidneys. It is one of the rare case because the incidence of DWM itself is $1 / 25.000$ birth. DWM charaterized by three finding: dilatation of fourth ventricle, enlargment of posterior cranial fossa, and agenenis or hipolasia of vermis cerebellum. Methods: We report a case of A 37 years old female in 24 week gestation age come to the hospital with persistently vaginal discharge due to leakage of vaginal fluid symptom. The onset was acute while the patient regularly did the antenatal care. The case is reviewed using relevant literatures to discuss about Coldston syndrome. Results: After referred to Intensive Care Unit (ICU) of Obstetric and Ginecology, ultrasound diagnsosis reveal Preterm Premature Rupture of Membrane(PPROM) and oligohydramnion or nil amniotic fluid. Vaginal discharge was found since 1 month before admission with no itchy, no fishy odor, and never been treated. Another finding of ultrasound examination were suitable with DWM and bilateral policystic kidney. As a result, Goldston syndrome was confirmed. The prognosis was explained to the patient and her family regarding the anomalous baby. Then, the termination of pregnancy was planned. The mother was in the good condition after that, while the baby died 10 minutes after delivered. Conclusion: Goldston syndrome is the rare cause of malformation which can be found during intrauterin or antenatal presentation. Ultrasound examination is the important tool to diagnose the disorder. Immediate treatment regarding the prognosis of the fetus is necessary to save the patient.

Keywords: Goldston syndrome, Dandy Walker Malformation, Premature. 
99.

\section{Liver's Functional Condition in Patients with Comorbid Course of Non-alcoholic Steatohepatitis and Asthma Depending on Body Mass Index}

Background: In recent years the incidence of bronchial asthma (BA) and non-alcoholic steatohepatitis (NASH) is growing rapidly. obesity has become epidemic as well. Quite often, timely diagnosis, efficient argumentation of treating the essential disease is complicated by the existing accompanying pathology that leads to the increased severity of its course, the formation of resistance to traditional treatments. Aim: To establish changes in the functional state of the liver in patients with NASH and obesity, depending on the presence of comorbid BA. Methods: 50 people aged 30 to 50 years (average age - 42) were examined, $40 \%$ of them - men and $60 \%$ - women. Among them in 30 patients with first-degree obesity (BMI over $30 \mathrm{~kg} / \mathrm{m}^{2}$ ) was diagnosed NASH, and in 20 other patients NASH was combined with first-degree obesity and persistent BA of medium severity. The duration of disease ranged from 2 to 6 years. Control group consisted of 20 almost healthy individuals, according to their age and sex. The examination embodied general clinical blood and urine analysis, rapid response to syphilis, glycemic blood profile, chest X-ray, ECG, scatological study, biochemical tests of liver function and ultrasound of the abdomen. Results: In patients of the second group the decreasing De Ritis ratio was revealed (AST / ALT) to $28.2 \%(p<0.05)$ and in the first group to $23.4 \%(p<0.05)$ in comparison to the USO. The authentic increase of total bilirubin's content in patients of the second group was established up to 2.2 times $(p<0.05)$ in comparison to the first-group patients, whose bilirubin's content has increased by 1.5 times. The maximum authentic thymol test indices also related to the second group ( $p$ $<0.05)$. The increased activity of alkaline phosphatase and $Y$-GT was found. It was $35.0 \%$ and $30.4 \%(p<0.05)$ in patients with NASH and BA together with obesity against $20.8 \%$ and $19.2 \%$ ( $p<0.05$ ) in patients of the first group. Bile acids' content in blood has also increased by 2.3 and 1.9 times ( $p<0.05$ ), indicating the presence of cholestasis, which was also observed in the second-group patients.

Conclusion: In patients with non-alcoholic steatohepatitis together with first-degree obesity and BA of moderate severity of the persistent flow, the content of markers, which are responsible for cytolysis activity of hepatocytes, cholestasis and mesenchymal inflammation, is increasing. It indicates the powerful impact of asthma on the course of NASH.

\section{Grand Theft Auto: Tension Pneumothorax}

JERNEJ BERGANT ${ }^{*}$, MD, Jost Jansa ${ }^{2}, \mathrm{MD}$

1 University clinical centre Ljubljana, Slovenia, ${ }^{2}$ General hospital lesenice, Slovenia

Background: Pneumothorax is defined as the presence of air between parietal and visceral pleural cavity. Tension pneumothorax is the accumulation of air under pressure in the pleural space. Case presentation: It was a peaceful night for 17y.0. boy until he was caught trying to steal Renault Clio. He was punched in the face and kicked several times in right thorax and abdomen. He came to the emergency department complaining about mild dyspnoea and painful head and right thorax. He had lacerated eyebrow and multiple subcutaneous hematomas on lateral side of right thorax, which had crackling feel to the touch and ribs were painful on palpation. Right lungs were hypersonoric on percussion and quiet on auscultation. He was hemodynamically stable with BP $134 / 76 \mathrm{mmHg}$, pulse $87 / \mathrm{min}$, respiration rate (RR) $16 /$ min. X-ray showed 5th - 7th rib fracture, moderate pneumothorax with no shift of structures and vast subcutaneous emphysema spreading into neck. $100 \%$ oxygen was administered to accelerate the rate of pleural air absorption. While waiting for chest drainage to be done, his dyspnoea gradually increased, $\mathrm{BP}$ was $92 / 52 \mathrm{mmHg}$, pulse $122 / \mathrm{min}$, RR $28 / \mathrm{min}$. Right lungs were quiet, more hypersonoric, neck veins were full. We suspected tension pneumothorax and immediately performed needle thoracocentesis. Air hissed and dyspnoea was relieved. Shortly after chest drainage was placed and he was admitted to ITU. Conclusion: While waiting for definitive treatment of pneumothorax (chest drainage) patients should be carefully monitored even if tension pneumothorax had been previously excluded. This condition if unrecognized and untreated, rapidly progresses to respiratory insufficiency, cardiovascular collapse and death. Favourable patient outcome requires urgent diagnosis and immediate decompression with $14-16 \mathrm{G}$, at least $45 \mathrm{~mm}$ long needle in 2nd or 3rd intercostal space in midclavicular line. A high index of suspicion and knowledge of basic emergency thoracic decompression is important for all healthcare personnel.

Keywords: tension, pneumothorax, thoracocentesis, drainage 